Salvador Homce de Cresce

\title{
ANÁLISE NÃO-LINEAR DE PAVIMENTOS DE CONCRETO ARMADO PELO MÉTODO DOS ELEMENTOS DE CONTORNO
}

Tese apresentada à Escola de Engenharia de São Carlos, da Universidade de São Paulo como parte dos requisitos para a obtenção do Título de Doutor em Engenharia de Estruturas

Orientador: Prof.Tit. Wilson Sergio Venturini

São Carlos

2003 
AUTORIZO A REPRODUÇÃO E DIVULGAÇ̃̃O TOTAL OU PARCIAL DESTE TRABALHO, POR QUALQUER MEIO CONVENCIONAL OU ELETRÔNICO, PARA FINS DE ESTUDO E PESQUISA, DESDE QUE CITADA A FONTE.

Ficha catalográfica preparada pela Seção de Tratamento da Informação do Serviço de Biblioteca - EESC/USP

c919a

Cresce, Salvador Homce de

Análise não-linear de pavimentos de concreto armado pelo método dos elementos de contorno / Salvador Homce de Cresce ; orientador Wilson Sergio Venturini. -- São Carlos, 2003.

Tese (Doutorado-Programa de Pós-Graduação e Área de Concentração em Engenharia de Estruturas) - - Escola de Engenharia de São Carlos da Universidade de São Paulo, 2003.

1. Métodos dos elementos de contorno. 2. Análise não linear. 3. Placas. I. Título. 
À minha esposa Léia e aos meus filhos Renata, Claudia e Fernando

À minha mãe Yvone 


\section{AGRADECIMENTOS}

Ao Professor Wilson Sergio Venturini, amigo e colega há mais de trinta anos, pela orientação e presença constantes.

Aos Professores do Departamento de Estruturas da EESC, pela amizade e pelos conhecimentos que me foram transmitidos.

Aos colegas da pós-graduação, jovens, amigos, simpáticos e sempre prestativos. 


\section{RESUMO}

CRESCE, S. H. Análise não linear de pavimentos de concreto armado pelo método dos elementos de contorno. São Carlos, 2003. Tese (Doutorado) - Escola de Engenharia de São Carlos - USP

Este trabalho trata da formulação do Método dos Elementos de Contorno para a análise não linear de pavimentos de concreto armado. A teoria utilizada é a de Reissner, que mostrou-se eficiente tanto para placas esbeltas quanto para as moderadamente espessas. Considera-se a ocorrência de cargas concentradas, distribuídas em sub-regiões da placa e em linha. Admite-se também a possibilidade de um campo de momentos iniciais, que viabiliza o estudo da não linearidade física nos problemas.

Foram utilizados campos de momentos iniciais aplicados apenas em pontos internos ao domínio.As integrais que envolvem as células de domínio foram modificadas, eliminando-se os núcleos complexos e as aproximações através de séries. Foi desenvolvida uma formulação para a análise de placas vinculadas a estruturas quaisquer em seu domínio, com o uso de cargas aplicadas incógnitas atuando como enrijecedores. O acoplamento MEC/MEF foi empregado utilizando-se modelos simples, porém robustos.O sistema de equações algébricas foi otimizado com a utilização da técnica dos mínimos quadrados.

O concreto foi modelado adotando-se o modelo de dano de Mazars; para as armaduras um modelo elastoplástico uniaxial com endurecimento isótropo. A análise não linear do problema é efetuada utilizando-se procedimento incremental-iterativo.

São apresentados alguns exemplos simples que mostram a precisão da técnica usada.

Palavras-chave: Método dos Elementos de Contorno, Análise não linear, Placas 


\begin{abstract}
CRESCE, S. H. Non-linear analysis of reinforced concrete bulding floors by the boundary element method. São Carlos, 2003. Tese (Doutorado) - Escola de Engenharia de São Carlos - USP

This work refers to the formulation of the boundary element method for nonlinear analysis of building floor structures. The plate bending theory adopted to develop the work wad due to Reissner, which has demonstrated to be efficient for thick, moderated thick and thin plates. The kinds of load applied on the plate medium surface have been taken into account: concentrated loading, distributed over sub-domains; distributed along internal lines. The presence of initial moment fields convenient to model temperature effects and to be used to build up non-linear solutions has also been considered in the formulation.
\end{abstract}

The domain integrals containing complex kernels to take into account the initial moment field influences were modified by introducing their primitive functions, avoiding therefore using series expansions. To integrate the initial moments fields only approximations based on internal nodal points were used. The resulting cell integrals have been transformed to the cell boundary which results into regular integral only.

A boundary element formulation to treat structural system defined by combining plates with other structural element was developed, using interface force as unknowns. The BEM/FEM coupling developed to treat this case is simple but robust; only displacements have been coupled avoiding important singularities that may happen when coupling rotations. The resulting system of algebraic equations has been regularized by using the least square method.

The concrete material was modeled by using the Mazar's damage model, while the steel reinforcement was assumed to behave as elastoplastic material with isotropic hardening. 
Finally, some examples are shown to illustrate the accuracy of the presented formulation and the numerical schemes proposed in this work.

Keywords: Boundary Element Method, Non-linear Analysis, Plates 


\section{SUMÁRIO}

1 INTRODUÇÃO .........................................................................................1

1.1 CONSIDERAÇÕES GERAIS ......................................................... 1

1.2 ORGANIZAÇÃO DO TRABALHO ................................................

1.3 REVISÃO BIBLIOGRÁFICA …...................................................4

1.3.1 TEORIA DAS PLACAS ...................................................................... 4

1.3.2 MÉTODOS NUMÉRICOS PARA ANÁLISE ESTRUTURAL ................ 6

1.3.3 O MÉTODO DOS ELEMENTOS DE CONTORNO NA ANÁLISE DE PLACAS 8

1.3.3.1 TEORIA CLÁSSICA DE KIRCHHOFF ………….......................... 8

1.3.3.2 TEORIAS DE REISSNER E MINDLIN .............................................. 10

1.3.3.3 MODELOS CONSTITUTIVOS PARA O CONCRETO ARMADO .... 12

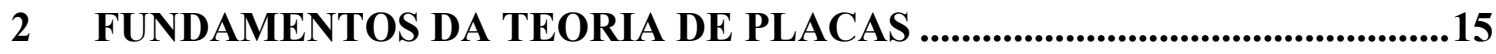

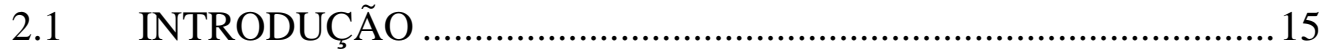

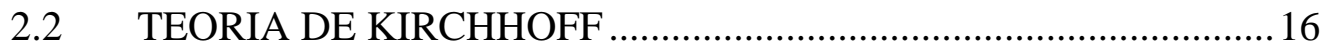

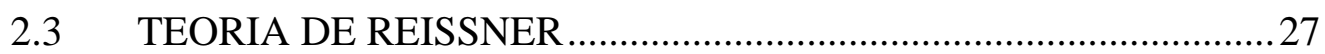

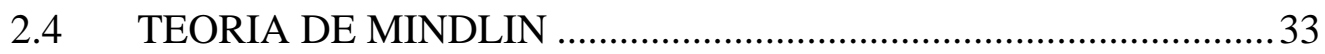

2.5 CAMPOS DE MOMENTOS INICIAIS ...........................................38

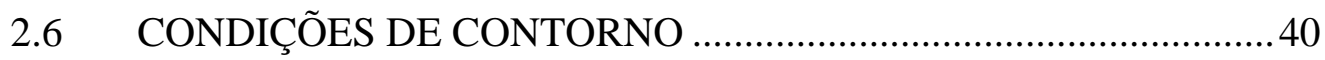

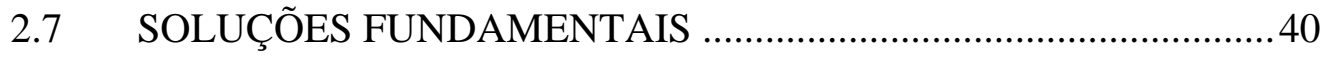

2.7.1 SOLUÇÃO FUNDAMENTAL PARA A TEORIA DE KIRCHHOFF .. 41

2.7.2 SOLUÇÃO FUNDAMENTAL PARA A TEORIA DE REISSNER ....... 42

3 EQUAÇÕES INTEGRAIS DE PLACAS - TEORIA DE REISSNER................45

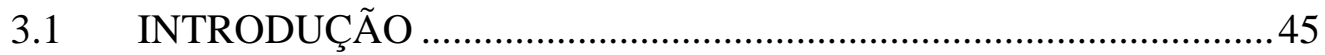

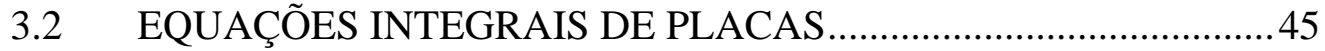

3.2.1 EQUAÇÃO INTEGRAL PARA DESLOCAMENTOS DE PONTOS DO INTERIOR DA PLACA 46

3.2.2 EQUAÇÃO INTEGRAL PARA DESLOCAMENTOS DE PONTOS DO CONTORNO DA PLACA. 47

3.2.3 EQUAÇÃO INTEGRAL PARA ESFORÇOS NOS PONTOS DO DOMÍNIO DA PLACA 48 
3.2.4 CARGAS E MOMENTOS DISTRIBUÍDOS NO DOMÍNIO DA PLACA 50

4 O MÉTODO DOS ELEMENTOS DE CONTORNO APLICADO À

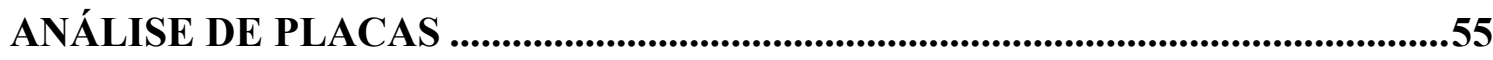

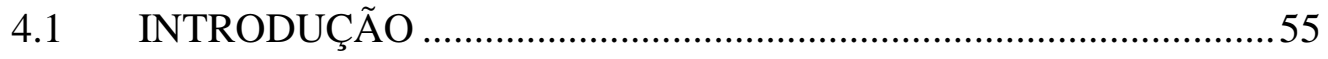

4.2 DISCRETIZAÇÃO DAS EQUAÇÕES INTEGRAIS.........................56

4.3 DESLOCAMENTOS NO CONTORNO DA PLACA .......................59

4.4 DESLOCAMENTOS EM PONTOS INTERNOS ............................62

4.5 ESFORÇOS EM PONTOS INTERNOS AO DOMÍNIO....................63

4.6 INFLUÊNCIAS DO CAMPO DE MOMENTOS INICIAIS ..............64

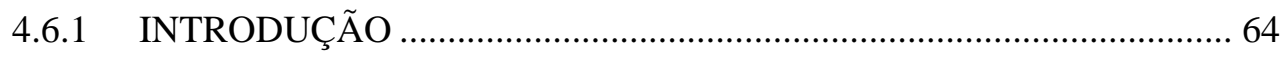

4.6.2 INFLUÊNCIA DOS MOMENTOS INICIAIS NOS DESLOCAMENTOS

4.6.3 INFLUÊNCIA DOS MOMENTOS INICIAIS NOS ESFORÇOS ........... 68

4.6.4 DETALHES DAS CÉLULAS EMPREGADAS .................................... 70

5 PLACAS COM ENRIJECEDORES - ACOPLAMENTO MEC/MEF.............73

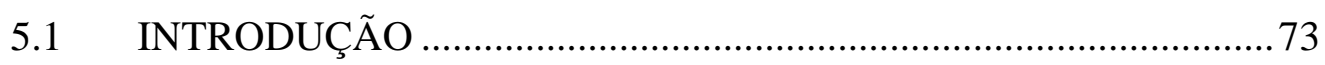

5.2 EQUAÇÃO INTEGRAL PARA PLACAS COM VÍNCULOS

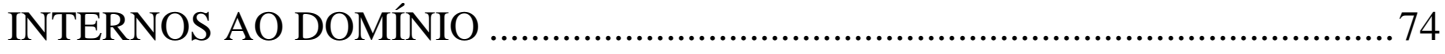

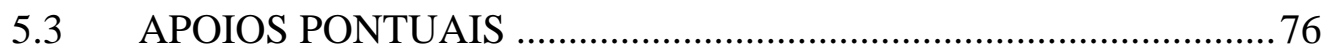

5.4 APOIOS EM ÁREAS DISCRETAS .................................................. 77

5.5 ASSOCIAÇÃO DA PLACA COM ESTRUTURA QUALQUER......82

5.6 ELEMENTOS FINITOS DE VIGA ..............................................91

5.7 FORMULAÇÃO DO ACOPLAMENTO MEC/MEF........................97

5.8 COMBINAÇÃO DAS EQUAÇÕES DO MEC/MEF COM REGULARIZAÇÃO PELO MÉTODO DOS MÍNIMOS QUADRADOS.................99

6 MODELOS CONSTITUTIVOS 103

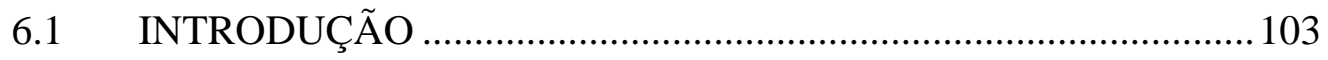

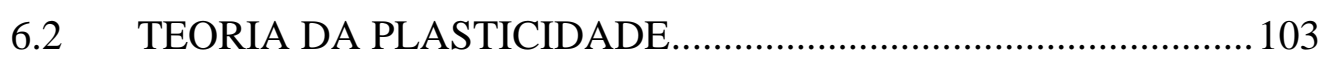

6.2.1 MODELO ELASTOPLÁSTICO PERFEITO........................................ 104

6.2.2 MODELO ELASTOPLÁSTICO COM ENCRUAMENTO - CASO UNIAXIAL 
6.2.3 MODELO ELASTOPLÁSTICO COM ENCRUAMENTO - CASO MULTIAXIAL 107

6.2.4 CRITÉRIO DE PLASTIFICAÇÃO DE VON MISES .......................... 112

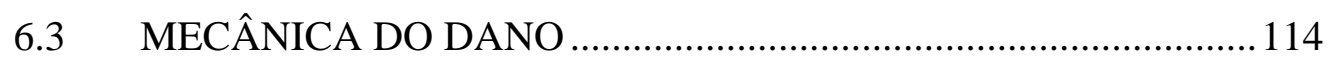

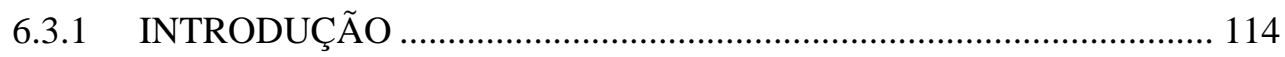

6.3.2 VARIÁVEL DE DANO .................................................................... 115

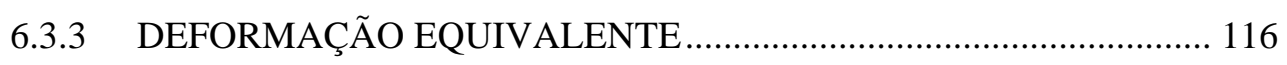

6.3.4 MODELO DE DANO DE MAZARS .................................................. 117

6.3.4.1 HIPÓTESES BÁSICAS ................................................................. 117

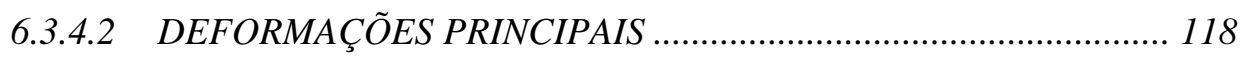

6.3.4.3 DEFORMAÇÃO EQUIVALENTE ............................................... 118

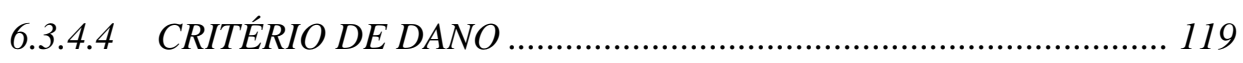

6.3.4.5 DETERMINAÇÃO DA VARIÁVEL DE DANO................................ 120

7 SOLUÇÃO DO PROBLEMA NÃO LINEAR .........................................................123

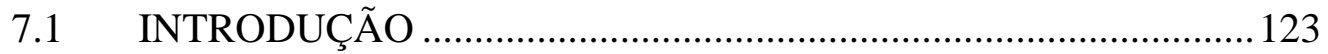

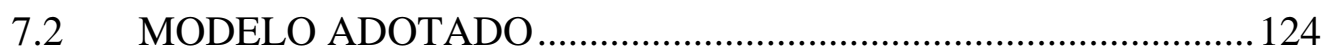

7.3 CÁlCULO DO MOMENTO INTERNO RESULTANTE NUMA

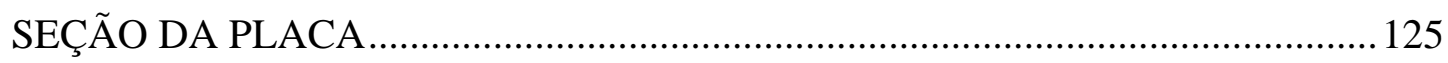

7.4 EQUILÍBRIO NA SEÇÃO DA PLACA ........................................126

7.5 PROCEDIMENTO INCREMENTAL ......................................... 131

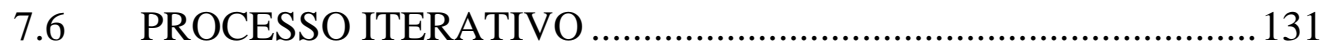

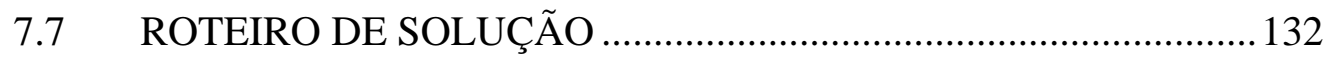

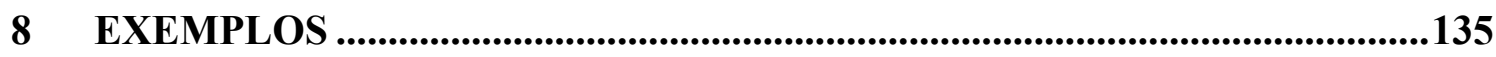

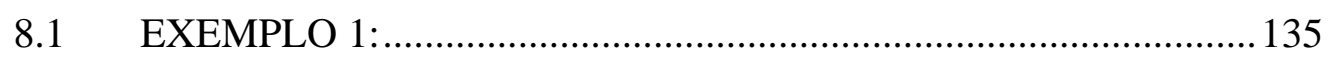

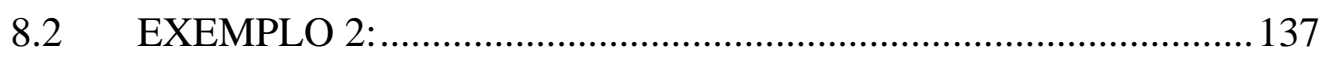

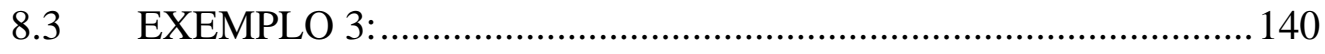

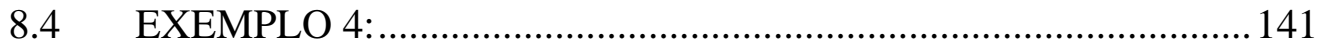

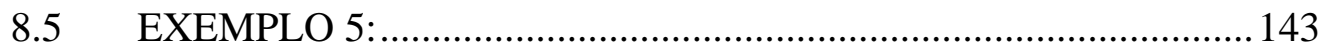

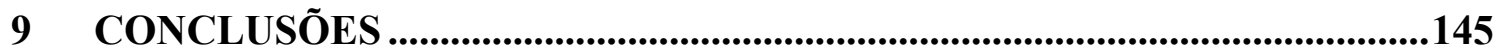

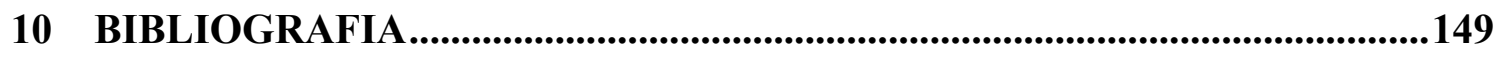

APÊNDICE - SOLUÇÕES FUNDAMENTAIS ...................................................... 157 


\section{INTRODUÇÃO}

\subsection{CONSIDERAÇÕES GERAIS}

No projeto estrutural de edifícios, o dimensionamento de pavimentos de concreto armado, constituídos de lajes e vigas aparece como sendo de fundamental importância, pois representa parte significativa dos custos da estrutura.

A laje tem seu comportamento representado teoricamente pelas placas, que são elementos estruturais cuja dimensão normal ao seu plano é pequena em relação às demais, e cujos carregamentos externos provocam solicitações normais à esse plano.

A análise das placas, com a obtenção dos esforços e deslocamentos em seus diversos pontos é objeto de diversas teorias, que são aproximações bidimensionais de problemas realmente tridimensionais.

As vigas e pilares podem ser representados por barras, cujos deslocamentos são iguais aos das lajes na interface com as mesmas. Podem ser considerados em modelos matemáticos como um enrijecimento localizado nas lajes.

A solução analítica destes problemas só é possível em casos simples, daí o seu estudo ser feito usualmente através de métodos numéricos aproximados, como o Método das Diferenças Finitas (MDF), o Método dos Elementos Finitos (MEF) e o Método dos Elementos de Contorno (MEC), cuja utilização prática só se tornou viável em tempos recentes com o advento e o barateamento do custo dos microcomputadores.

A formulação do cálculo de placas teve suas hipóteses formuladas inicialmente na chamada Teoria Clássica por Kirchhoff, aplicada principalmente às placas de 
pequena espessura, sendo posteriormente seguida pelas teorias de Reissner e Mindlin. $\mathrm{Na}$ análise de placas, as hipóteses de Reissner-Mindlin, que consideram os efeitos de força cortante, conduzem a bons resultados, mais precisos que os obtidos pela teoria clássica de Kirchhoff, particularmente no caso de lajes de grande espessura.

O Método dos Elementos de Contorno, por sua vez apresenta um bom desempenho na análise de placas, sendo sua precisão particularmente notada por HARTMANN (1988), levaram-no a sugerir que o mesmo supera o Método dos Elementos Finitos neste tipo de problema.

O concreto e o aço envolvidos tem seu comportamento estrutural representado neste trabalho por modelos que consideram as suas não-linearidades físicas, como a Teoria da Plasticidade e a Mecânica do Dano. Em muitos casos também poderia também ser considerada a não-linearidade geométrica das estruturas, o que aqui não foi realizado.

Considerando o grande número de hipóteses adotadas, muitas formulações têm sido experimentadas, sendo o grande desafio representado pela coerência entre os valores obtidos pela experimentação com os calculados por elas, a precisão neste caso é de grande importância. O esforço computacional envolvido tem progressivamente perdido sua importância à medida que aumenta a capacidade de processamento dos computadores, ao mesmo tempo em que diminui proporcionalmente o seu preço.

O objetivo deste trabalho é o cálculo de pavimentos de edifícios pelo Método dos Elementos de Contorno. As análises elásticas serão efetuadas segundo as hipóteses de Reissner - Mindlin. Os cálculos serão ampliados para permitir a solução de problemas com não-linearidade física dos materiais, utilizando-se procedimento incremental e iterativo. Para aumentar a precisão dos resultados foi adotado novo procedimento utilizando-se apenas esforços nos pontos internos como corretores dos momentos no procedimento incremental-iterativo.

São apresentadas algumas novas formulações, particularmente no caso de carregamentos de domínio, com vistas à obtenção de resultados mais precisos.

O acoplamento entre o Método dos Elementos de Contorno e o Método dos Elementos Finitos foi utilizado na interface entre as lajes, vigas e pilares do pavimento de concreto armado.Foi experimentada a utilização de um elemento de barra simples, 
transferindo apenas forças perpendiculares à placa, o que possibilitou robustez ao processo Mais adiante, é utilizado um sistema de equações super-abundantes, otimizados os resultados com a técnica dos mínimos quadrados.

Um programa em linguagem FORTRAN foi desenvolvido para efetuar os cálculos envolvidos. O objetivo ao final do trabalho é chegar a uma formulação aperfeiçoada que possibilite obter resultados mais precisos do que os hoje existentes.

\subsection{ORGANIZAÇÃO DO TRABALHO}

O capítulo 1 termina com uma revisão bibliográfica sobre os temas referentes a: Teoria de Placas, considerando-se as teorias de Kirchhoff, Reissner e Mindlin; o desenvolvimento dos métodos numéricos utilizados na engenharia estrutural atual; os modelos constitutivos utilizados para o concreto armado baseados na Teoria da Plasticidade e na Mecânica do Dano.

No capítulo 2 são estudados os fundamentos da Teoria de Placas com suas equações, seguida por comparativo entre as Teorias de Kirchhoff, Reissner e Mindlin, inclusive as relações entre suas soluções fundamentais.

O capítulo 3 é dedicado ao estudo das equações integrais referentes à Teoria de Reissner. Apresenta-se a representação integral dos deslocamentos para pontos do domínio, estendendo-se após para pontos do contorno. Obtêm-se as representações dos esforços nos pontos internos a partir das representações integrais dos deslocamentos. Analisam-se os termos de domínio referentes aos carregamentos aplicados em áreas, linhas, ou concentrados em pontos, bem como é estudado o efeito de campo de momentos iniciais com vistas a aplicação não-linear.

No capítulo 4, estuda-se a aplicação da teoria de Reissner à análise de placas, discretizando o contorno em elementos onde são aproximadas as funções que representam os deslocamentos e esforços. As equações integrais de contorno são transformadas em um sistema de equações algébricas lineares.

O capítulo 5 estuda a interação da placa com outros elementos estruturais como pilares e vigas. As vigas são analisadas como linhas de carga e o enrijecimento é 
calculado pelo método dos elementos finitos. A interação entre a placa e as vigas é representada por modelo simplificado de linhas de carga, considerando apenas cargas verticais. É obtido um sistema de equações redundante, feita a regularização pelo método dos mínimos quadrados.

No capítulo 6 são vistos os modelos constitutivos utilizados para o concreto e armadura, baseados na teoria da plasticidade e na mecânica do dano contínuo.

O processo de solução do problema não linear através de procedimento incremental e iterativo é tratado no capítulo 7.

No capítulo 8 são apresentados exemplos numéricos de cálculo, utilizando a formulação dada.

O capítulo 9 apresenta as conclusões do trabalho, bem como sugestões para sua continuidade.

\subsection{REVISÃO BIBLIOGRÁFICA}

\subsubsection{TEORIA DAS PLACAS}

Entende-se por placa um corpo delimitado por duas superfícies de pequena curvatura, a distância entre estas superfícies, chamada de espessura, sendo pequena em relação às dimensões da superfície e considerada constante. A superfície eqüidistante das faces externas é chamada de superfície média. As placas são solicitadas por esforços externos normais à superfície média.

A introdução de simplificações, visando analisar a placa como elemento bidimensional em vez de tridimensional originou diversas teorias. As mais usadas são a Teoria de Kirchhoff e a Teoria de Reissner/Mindlin.

O primeiro trabalho nesta área foi desenvolvido por KIRCHHOFF (1850) que elaborou a chamada Teoria Clássica que visa à análise de placas delgadas com pequenos deslocamentos, sob carregamento transversal. A formulação do problema conduz a uma equação diferencial de quarta ordem, onde devem ser satisfeitas duas condições de contorno ao longo dos limites da placa, desconsiderando-se as deformações por cisalhamento transversal. 
Alternativamente a esta teoria, REISSNER $(1944,1945)$ desenvolveu teoria que considera as deformações por cisalhamento transversal, obtendo-se um sistema de equações diferenciais de sexta ordem. Com este sistema podem ser verificadas três condições em cada ponto do contorno.

Modelo próximo ao de Reissner foi proposto por MINDLIN (1951) para placas fletidas elásticas e isotrópicas, baseado nas equações da teoria da elasticidade tridimensional para um corpo em movimento. A teoria também leva em consideração o cisalhamento transversal e o sistema de equações diferenciais obtido é de sexta ordem.

As teorias de Reissner e Mindlin apresentam resultados melhores que os da Teoria Clássica de Kirchhoff, principalmente para pontos situados nas bordas, além de permitirem a análise de placas delgadas e espessas.

SALERNO \& GOLDBERG (1960) reduziram o sistema de três equações diferenciais de Reissner para uma equação diferencial de quarta ordem, semelhante à da Teoria Clássica e a uma equação diferencial de segunda ordem para a determinação de uma função de tensão. Para placas simplesmente apoiadas, os resultados aproximam-se dos da Teoria Clássica.

PANC (1975) analisou diversas teorias para análise de placas.

NORDGREN (1971), (1972) afirmou que os valores calculados pela Teoria Clássica podem ser aceitos como aproximações de problemas tridimensionais correspondentes. Deduziu expressões que mostram que o erro quadrado médio da Teoria Clássica é proporcional à espessura da placa, enquanto que para a Teoria de Reissner é proporcional ao quadrado da espessura.

CHENG (1959) desenvolveu nova teoria com equação diferencial de ordem infinita, onde derivadas maiores que as de quarta ordem multiplicam os quadrados de espessura da placa. Quando a espessura da placa tende a zero, obtém-se a equação da Teoria Clássica.

LEVINSON (1980) apresentou teoria para análise dinâmica de placas, considerando as deformações por cisalhamento, obtendo neste caso resultados similares aos obtidos por Mindlin. 
REISSNER (1986) apresentou nova formulação para análise de placas delgadas submetidas a grandes deformações, obtendo um sistema de equações diferenciais de décima ordem.

No ano seguinte, REISSNER (1987) tratou do problema de placas moderadamente espessas, com sistema de equações diferenciais de décima segunda ordem, mostrando resultados consistentes com valores clássicos.

RYCHTER (1988) provou que a Teoria de Reissner combinada com o estado plano de tensão é capaz de avaliar o comportamento real de placas elásticas homogêneas, com erro relativo máximo da ordem do cubo da espessura.

BARRET \& ELLIS (1988) apresentaram nova formulação para placas submetidas a carregamento transversal, apresentando também os relacionamentos entre a sua teoria e as de Kirchhoff, Reissner e Mindlin.

\subsubsection{MÉTODOS NUMÉRICOS PARA ANÁLISE ESTRUTURAL}

Tendo em vista que as soluções analíticas das equações diferenciais dos problemas estruturais correntes são raras, criaram-se técnicas numéricas para obtenção de soluções aproximadas, soluções estas que tiveram grande incremento nas últimas duas décadas decorrente dos grandes avanços da informática.

Estes métodos dividem-se em dois grandes grupos:

Métodos de domínio, onde na solução das equações diferenciais são utilizadas aproximações dos valores de variáveis associadas em pontos do domínio e do contorno das peças. Pertencem a este grupo os métodos das Diferenças Finitas (MDF) e dos Elementos Finitos (MEF).

Métodos de contorno, onde na solução das equações diferenciais são utilizadas aproximações dos valores de variáveis associadas, apenas em pontos do contorno das peças. Pertence a este grupo o método dos Elementos de Contorno (MEC).

O Método das Diferenças Finitas é o primeiro método de tratamento numérico de problemas de engenharia formulado em bases consistentes e bastante usado até hoje. Surgiu com o trabalho de SOUTHWELL (1946), consiste na transformação das equações diferenciais em um sistema de equações lineares através da aplicação de 
operadores algébricos que calculam as derivadas como funções dos seus valores em pontos próximos, através aproximações lineares.

O Método dos Elementos Finitos (MEF) surgiu unindo o tratamento matricial de reticulados com o método da energia, o domínio é dividido em células, cada qual com pontos característicos (nós), aos quais são atribuídos valores para representar as incógnitas do problema. Estes valores são avaliados nas células por funções aproximadoras, em geral polinomiais. Em muitos casos se faz necessário a subdivisão da peça em grande número de células para a obtenção de resultados mais precisos. Deu forte contribuição ao estudo de técnicas variacionais e de resíduos ponderados.

Os trabalhos iniciais em placas foram desenvolvidos por TURNER (1956) e ARGYRIS \& KELSET (1960), basearam-se nas hipóteses de Kirchhoff, com funções aproximadoras de classe C1(função e primeira derivada contínuas).

As evoluções ocorridas até então foram apresentadas nos trabalhos de BATOZ et al. (1980) que adotaram modelo em deslocamentos com hipóteses de Kirchhoff de forma discretizada ao longo dos lados dos elementos. Conseguiram bom desempenho com elemento denominado DKT (Discrete Kirchhoff Triangle).

Grande evolução ocorreu quando começaram a usar as hipóteses de Reissner em lugar das de Kirchhoff, para placas moderadamente espessas. Vantagem importante nesta mudança está no fato de serem exigidas funções aproximadoras para deslocamentos com continuidade classe C0, por serem derivadas de primeira ordem as mais altas que aparecem no funcional, além do fato de ser considerado o cisalhamento transversal.

Após alguns problemas encontrados na fase inicial, houve evolução nos trabalhos de TESSLER \& HUGHES (1985) que trabalhou com elemento triangular com as hipóteses de Mindlin, PAPADOPOULOS \& TAYLOR (1990) trabalharam com as teorias de Reissner/Mindlin em elementos triangulares.

O método numérico denominado Método dos Elementos de Contorno (MEC), derivado do tratamento das equações integrais de contorno, foi assim denominado por BREBBIA (1978). Nasceu dos estudos do MEF e ao invés de resolver as equações diferenciais no domínio do problema, procura resolver as equações integrais no 
contorno associadas às mesmas. As variáveis do problema são calculadas em pontos discretos do contorno e, a partir desses valores, calculam-se os valores no domínio.

Apresenta como vantagem a redução de uma dimensão do problema, já que a discretização é feita apenas no contorno, ao invés de ser feita na área como no MEF, levando a uma quantidade menor de dados de entrada. Outras vantagens seriam a representação de domínios infinitos, a determinação de valores em pontos internos ao domínio sem a necessidade de interpolações no domínio e sem alterar a discretização do contorno.

Apesar de ser o mais recente, o Método dos Elementos de Contorno originou-se na resolução de equações integrais, conhecidas já no século XIX. Historicamente a primeira aplicação de equações integrais à Teoria da Elasticidade foi devida a BETTI (1872).

Foi seguido por MUSKHELISSHVILI (1953), MIKHLIN (1957) e KUPRADZE (1965) que trabalharam com o chamado método indireto, onde as variáveis envolvidas não eram as variáveis reais do problema.

O método direto, onde as variáveis físicas do problema coincidem com as apresentadas na formulação, foi inicialmente apresentado por RIZZO (1967) para problemas de elasticidade bidimensional.

BREBBIA (1978) obteve as equações integrais a partir da técnica dos resíduos ponderados, associando o método aos outros métodos numéricos. A partir dele ocorreu grande impulso na utilização do MEC em diversos problemas de engenharia, como a consideração de não linearidade física e geométrica, a interação solo-estrutura, etc.

\subsubsection{O MÉTODO DOS ELEMENTOS DE CONTORNO NA ANÁLISE DE PLACAS}

\subsubsection{Teoria Clássica de Kirchhoff}

O trabalho inicial do MEC em placas foi de JASWON et al. (1967) baseado na Teoria Clássica de Kirchhoff, propondo a decomposição da equação bi-harmônica em duas equações diferenciais harmônicas, transformando as mesmas em equações integrais no contorno e obtendo a solução, pelo método indireto.

FORBES \& ROBINSON (1969) foram os primeiros a utilizar o método direto. 
HANSEN (1976) utilizou a formulação direta para análise de placas infinitas com furos de contorno não carregado com duas equações integrais calculando o deslocamento transversal e sua derivada numa direção qualquer.

BEZINE \& GAMBY (1978), desenvolveram formulação direta para a análise de placas partindo da identidade de Green e considerando duas equações integrais para o deslocamento transversal e sua derivada normal ao contorno.

BEZINE (1978) utilizou formulação direta, discretização com elementos constantes, sem transformar a integral de domínio em integral de contorno, seu estudo ficou desta forma restrito às cargas concentradas.

ALTIERO \& SIKARSKIE (1978) desenvolveram formulação indireta onde consideram a placa real contida numa outra fictícia, na qual a função de Green é conhecida, analisaram apenas placas engastadas.

STERN (1979) também utilizou formulação direta, discretização com elementos com aproximação linear, incluiu o cálculo das reações de canto como função dos momentos volventes, não considerou a hipótese de descontinuidade das condições de contorno nos nós de canto.

BEZINE (1981) desenvolveu formulação para placas com apoios dentro do domínio.

MOSHAIOV \& VORUS (1986) estudaram o comportamento elastoplástico usando carregamento incremental com a consideração de momentos fletores iniciais calculados por processo iterativo. Foram utilizadas células no domínio e admitido momento constante em cada uma delas.

PAIVA (1987) estendeu o método dos elementos de contorno para estruturas formadas por placas, vigas e pilares. Os elementos de vigas e pilares são equacionados pelo método dos deslocamentos, sendo as respectivas relações, juntamente com as condições de equilíbrio e compatibilidade de deslocamentos introduzidas no sistema de equações do MEC.

HARTLEY \& ABDEL-AKHER (1989) apresentaram proposta para a solução de singularidades utilizando a integração analítica de funções na determinação de valores referentes aos pontos internos. 
KARAMI et al (1992) desenvolveram formulação utilizando uma equação harmônica e uma equação bi-harmônica sendo as integrais de contorno calculadas analiticamente.

CHUEIRI (1994) tratou da análise elástica de placas, completou-a com a inclusão de campo de deformações iniciais, estendendo-a para a análise elastoplástica. Incluiu modelo para aplicação em lajes de concreto armado.

OLIVEIRA NETO (1998) utilizou três parâmetros nodais de deslocamentos e dois valores nodais para esforços, obtendo três equações integrais de contorno por nó.

FERNANDES (1998) estudou a análise não linear de placas de concreto armado utilizando modelo de dano para o concreto e modelo elastoplástico unidimensional para o aço.

\subsubsection{Teorias de Reissner e Mindlin}

A consideração do problema de placas usando a teoria de Reissner foi inicialmente efetuada por WEEËN (1982). Estabeleceu três equações integrais por nó do contorno, representando as relações entre os deslocamentos (deslocamento transversal, rotações normal e paralela ao contorno) e os esforços correspondentes (força cortante, momentos normal e tangencial), adotando elementos quadráticos na aproximação do contorno.

KARAM (1986) pesquisou na mesma linha de Weeën demonstrando a eficiência das hipóteses de Reissner com diversos exemplos de placas isotrópicas em regime elástico linear.

RIBEIRO \& VENTURINI (1989) escreveram o sistema de equações usando pontos fonte fora do domínio para evitar a ocorrência de singularidades.

XIAO-YAN et al. (1990) consideraram a não linearidade geométrica devido à ocorrência de grandes deslocamentos, utilizando um sistema iterativo para a linearização das equações.

RIBEIRO (1992) considerou a ocorrência de cargas concentradas, distribuídas em sub-áreas e em linha. Utilizou elementos de contorno de geometria linear e aproximação parabólica para as variáveis de contorno. 
KARAM (1992) e RIBEIRO (1992) apresentaram formulação para não linearidade física, nos mesmos moldes da proposta por Vorus para a Teoria Clássica.

KATSIKADELIS \& YOTIS (1993) propuseram solução alternativa para o tratamento da teoria de Reissner e partindo das equações diferenciais apresentadas em TIMOSHENKO \& WOINOWSKY-KRIEGER (1959), obteve solução que continha a soma de dois potenciais, um de Bessel e um bi-harmônico. O método depende da solução de três equações integrais e três equações de diferenças finitas.

DEBBIH et al. (1995) apresentaram solução fundamental modificada de forma que as partes das funções representativas do efeito das tensões transversais foram separadas permitindo análise de placas finas e espessas. Afirma que para a solução de placas finas os termos que corrigem o efeito da força cortante deveriam ser removidos da solução fundamental, para um melhor resultado numérico, ou seja, nestes casos foi entendido ser mais precisa a Teoria Clássica.

SILVA (1996) formulou a análise de placas com vigas e pilares, considerados como enrijecedores, a interação entre a laje e as vigas feita com combinação dos métodos MEF e MEC.

ALIABADI et al. (1997) trataram da avaliação das tensões na placa, através de dois métodos, o primeiro baseado nas tensões e deformações locais, o segundo baseado na avaliação direta do tensor de tensões, usando a equação integral de tensão.

PALERMO (2000) mostrou a conexão entre a Teoria Clássica e a Teoria de Mindlin, escrevendo as equações diferenciais desta com termos equivalentes aos usados nos estados planos através de rotações e dilatações. Obteve solução fundamental igual à obtida por Weeën e também a solução da Teoria Clássica, permitindo entender melhor a natureza das correções que foram introduzidas pelas teorias de Reissner e Mindlin.

BACARJI (2001) desenvolveu modelo para análises de pavimentos de concreto armado em edifícios, com ênfase em lajes cogumelo. Analisou-se o caso de campo de momentos iniciais para o estudo de problemas de não-linearidade física.

CARMO (2001) estudou pavimentos de edifícios através de combinação MECMEF considerando o efeito da excentricidade do eixo neutro das barras em relação à superfície neutra da placa, acrescendo-o ao fenômeno da flexão da placa. Utilizou a técnica das sub-regiões para o acoplamento dos elementos. 
BOTTA (2003) desenvolveu formulação não-linear com o MEC para análise numérica de sólidos danificados, considerando-se o fenômeno de localização de deformações, usando dois modelos de dano para o concreto. Considerou o acoplamento do MEC com o MEF para modelar o meio contínuo com fibras.

\subsubsection{Modelos Constitutivos para o Concreto Armado}

Diversos tipos de modelos constitutivos tem sido utilizados para descrever o comportamento das peças de concreto como modelos elastoplásticos, modelos de dano, de fratura e outros.

KACHANOV (1958) introduziu o conceito de dano para modelar a fissuração distribuída na ruptura frágil dos metais após serem submetidos à deformação lenta.

CERVENKA (1970) aplicou a teoria da plasticidade ao concreto comprimido, com critérios análogos aos usados nos materiais metálicos.

JOFRIET \& McNEICE (1971) consideraram efeito de fissuração, adotaram relação bilinear para momento-curvatura para representar o comportamento do concreto.

RAO \& SUBRAHMANYAN (1973) propuseram relação trilinear momentocurvatura e consideraram a resistência do concreto tracionado entre duas fissuras.

CHEN \& CHEN (1975) adotaram estado tridimensional de tensão para o concreto, considerando-o como material elastoplástico com endurecimento.

KRAJCINOVIC \& FONSEKA (1981) propuseram modelo vetorial de dano, dado em função das coordenadas do ponto e de variáveis de estado.

MAZARS (1984) apresentou modelo escalar de dano, adequado para carregamentos crescentes. A variável de dano é calculada como função de deformações equivalentes, calculadas localmente em função dos alongamentos do material. Os parâmetros do modelo são ajustados em função de resultados experimentais.

PROENÇA (1988) fez uma análise detalhada sobre vários modelos constitutivos para o concreto.

HU \& SCHNOBRICH (1991) adotaram para o aço comportamento elastoplástico perfeito e para o concreto modelo elastoplástico com endurecimento na compressão e tensão limitada à tração. 
ALVARES (1993) estudou modelo de dano para o concreto com identificação experimental de parâmetros, usando o MEF.

BUSSAMRA (1993) apresentou equações constitutivas para o concreto baseadas na mecânica do dano contínua.

No Departamento de Estruturas da Escola de Engenharia de São Carlos foram desenvolvidas algumas dissertações e teses onde se focalizaram algumas aplicações de modelo de dano às estruturas de concreto armado. Podem ser citados BOTTA (1998). PITUBA (1998), ALVARES (1999) e DRIEMEIER (1999). 


\section{FUNDAMENTOS DA TEORIA DE PLACAS}

\subsection{INTRODUÇÃO}

Placas são elementos estruturais planos, simétricos em relação a um plano médio, cuja dimensão normal a esse plano médio (espessura) é pequena em relação às dimensões da superfície, e que são solicitadas por esforços externos normais a esse plano médio.

Considerando as propriedades da placa podemos classificá-la em: ortótropa, com propriedades diferentes em duas direções ortogonais de sua superfície ou isótropa, com propriedades iguais em todas as direções.

Quanto à espessura, chamando-a de $\underline{\mathrm{d}}$ e denominando de a o comprimento do menor lado da placa, temos:

Placas muito delgadas para $\mathrm{d} / \mathrm{a}<1 / 100$;

Placas delgadas para d/a situado entre $1 / 5$ a 1/100 onde o valor $1 / 10$ pode ser encontrado, por exemplo, em pontes de laje e valores da ordem de 1/80 para lajes de forro de edifícios;

Placas espessas para $\mathrm{d} / \mathrm{a}>1 / 5$.

A Teoria de Kirchhoff não permite avaliar o efeito da espessura sobre os valores dos deslocamentos e esforços, considerando a placa como delgada. A Teoria de Reissner permite esta avaliação e pode ser usada tanto para placas delgadas como espessas. As formulações para placas muito delgadas devem considerar grandes deslocamentos. 


\subsection{TEORIA DE KIRCHHOFF}

Interpreta com precisão o problema das placas delgadas com pequenos deslocamentos, tem como hipóteses básicas:

- O material da placa é homogêneo, isotrópico e elástico-linear;

- Uma reta normal à superfície média inicialmente indeformada, permanece normal à mesma superfície após a deformação sofrendo apenas rotação, isto é, não são consideradas as deformações por cisalhamento transversal. Além disso, as deformações variam linearmente em função de sua distância ao plano médio;

- As tensões aplicadas nas superfícies externas são muito pequenas em relação às tensões normais de flexão, paralelas ao plano médio, podendo ser desprezadas;

- Não ocorrem deformações no plano médio da placa.

OBSERVAÇÃO: O fato de desprezar as tensões normais à superfície da placa coincide com a maior parte dos casos reais, por exemplo, conforme FUNG (1965), a pressão aerodinâmica atuando nas asas de um avião varia de 1 a 10 libras por polegada quadrada enquanto que os esforços na direção da "pele” da asa chegam a valores de 10.000 a 200.000 libras por polegada quadrada. Esta situação prática é importante para a simplificação da teoria.

A partir destas hipóteses, e considerando a convenção de sinais dada na figura abaixo, podem ser deduzidas as seguintes relações:

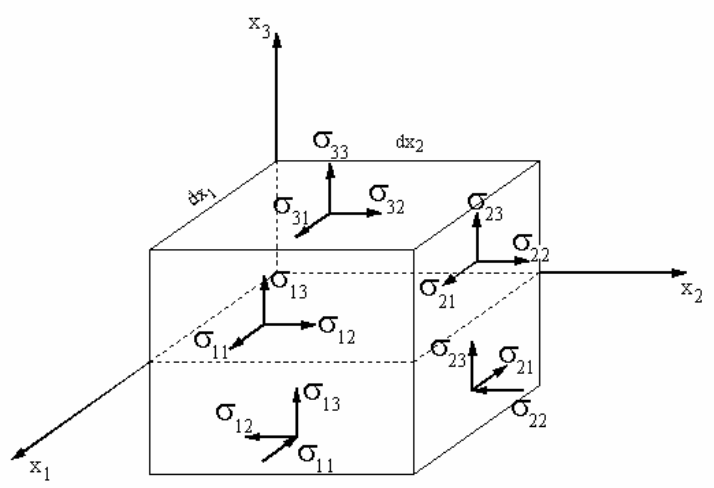

Figura 2.1 - Elemento de Placa e Componentes de Tensão 
Considerando a quarta hipótese temos:

$$
\begin{gathered}
\sigma_{z x}=0, \quad \sigma_{z y}=0 \ldots \ldots \ldots . . . . . \\
\sigma_{z z}=0, \quad-h / 2 \leq z \leq h / 2
\end{gathered}
$$

Da primeira e terceira hipótese vem:

$$
\sigma_{x x}=b_{1}(x, y) z, \quad \sigma_{y y}=b_{2}(x, y) z
$$

Adotam-se as seguintes convenções de sinais (ver figura 2.2):

As tensões normais $\sigma$ são positivas quando provocam tração na parte inferior do elemento, as tensões tangenciais são positivas, tomando a parte inferior do elemento como referência, quando coincidem com o sentido positivo dos eixos;

Os momentos fletores são positivos se provocam tração na fibra inferior;

Os momentos volventes são positivos quando seu vetor é emergente da face considerada;

As forças cortantes são positivas se, olhando o eixo crescente da esquerda para a direita, tendem a girar o elemento no sentido horário.

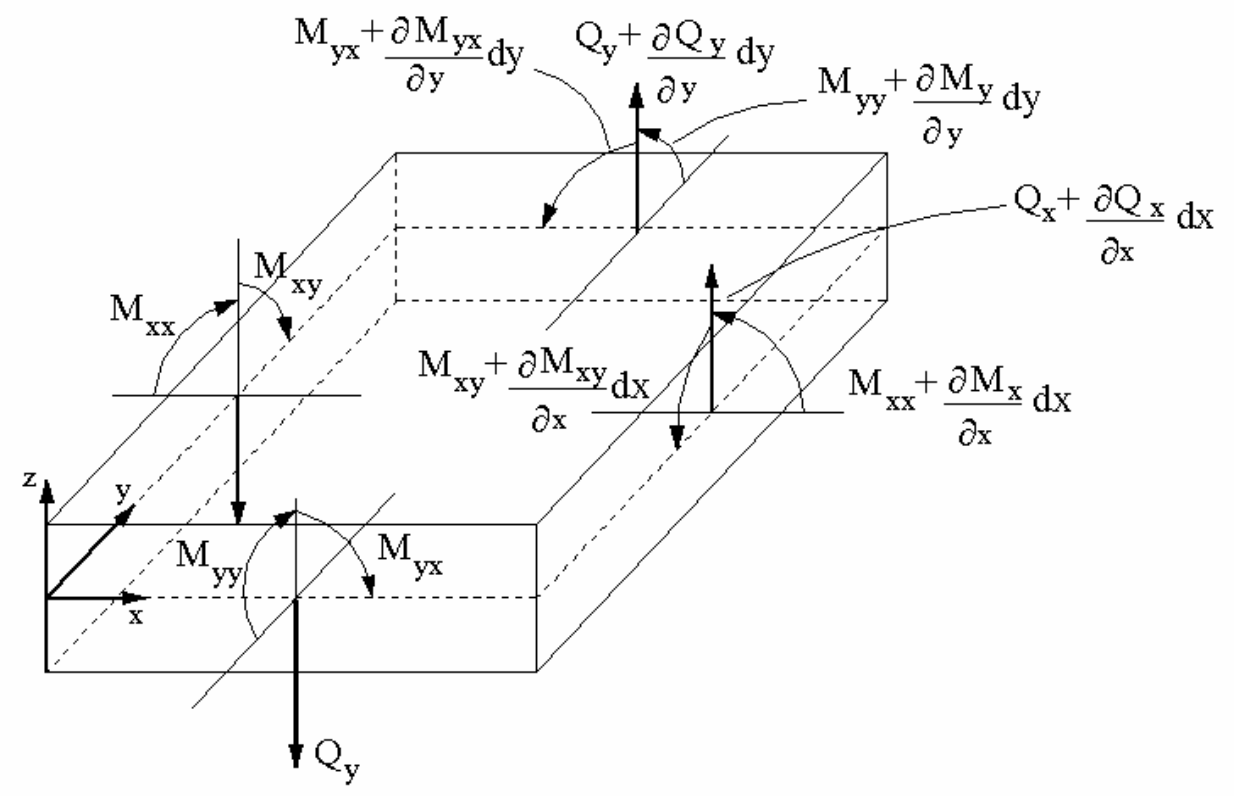

Figura 2.2 - Convenção de sinais para momentos e cortantes 
Considerando a placa submetida apenas a pequenos deslocamentos $\mathrm{u}_{\mathrm{i}}$, o tensor de deformações pode ser dado por ${ }^{1}$ :

$$
\varepsilon_{i j}=\frac{1}{2}\left(\frac{\partial u_{i}}{\partial x_{j}}+\frac{\partial u_{j}}{\partial x_{i}}\right)
$$

De acordo com a hipótese admitida de comportamento elástico linear, a relação tensão-deformação (lei de Hooke) é dada por:

$$
\sigma_{i j}=\frac{2 G v}{1-2 v} \varepsilon_{l l} \delta_{i j}+2 G \varepsilon_{i j}
$$

ou a relação inversa deformação-tensão dada por:

$$
\varepsilon_{i j}=\frac{1}{2 G}\left(\sigma_{i j}-\frac{v}{1+v} \sigma_{l l} \delta_{i j}\right)
$$

sendo:

$$
G=\frac{E}{2(1+v)}
$$

onde:

$$
\begin{aligned}
& E=\text { módulo de elasticidade longitudinal (Young) } \\
& G=\text { módulo de elasticidade transversal } \\
& v=\text { coeficiente de Poisson }
\end{aligned}
$$

Considerando (2.1), (2.2) e (2.4) valem as seguintes relações:

\footnotetext{
${ }^{1}$ Usamos índices com letras i,j,k variando de 1 a 3, letras gregas alfa,beta variando de 1 a 2 .
} 


$$
\begin{aligned}
& \varepsilon_{z x}=\frac{1}{2}\left(\frac{\partial u_{x}}{\partial z}+\frac{\partial u_{z}}{\partial x}\right)=0 \\
& \varepsilon_{z y}=\frac{1}{2}\left(\frac{\partial u_{y}}{\partial z}+\frac{\partial u_{z}}{\partial y}\right)=0 \\
& \varepsilon_{z z}=\frac{\partial u_{z}}{\partial z}=-\frac{v}{E}\left(\sigma_{x x}+\sigma_{y y}\right) .
\end{aligned}
$$

Equações (2.9), (2.2) e (2.3) juntas conduzem a:

$$
\left.u_{z}=w(x, y)-\frac{v}{E}\left(b_{1}(x, y)+b_{2}(x, y)\right)\right) \frac{z^{2}}{2}
$$

onde a função $\mathrm{w}(\mathrm{x}, \mathrm{y})$ representa o deslocamento vertical do plano médio da placa. Em vista da pequena espessura da placa, o segundo termo em (2.10) é em geral pequeno e pode ser desprezado em comparação com o primeiro termo. Adotando w(x,y) em lugar de $\mathrm{u}_{\mathrm{z}}$ em (2.8), e integrando, obtemos:

$$
\begin{aligned}
& u_{x}=-z \frac{\partial w(x, y)}{\partial x} \\
& u_{y}=-z \frac{\partial w(x, y)}{\partial y}
\end{aligned}
$$

onde $\mathrm{u}(\mathrm{x}, \mathrm{y}), \mathrm{v}(\mathrm{x}, \mathrm{y})$ e $\mathrm{w}(\mathrm{x}, \mathrm{y})$, a partir deste ponto, serão representadas por $\mathrm{u}, \mathrm{v}$ e $\mathrm{w}$, respectivamente.

De (2.11) deduzem-se as relações que fornecem as deformações: 


$$
\begin{aligned}
& \varepsilon_{x x}=\frac{\partial u}{\partial x}=-z \frac{\partial^{2} w}{\partial x^{2}} \\
& \varepsilon_{y y}=\frac{\partial v}{\partial y}=-z \frac{\partial^{2} w}{\partial y^{2}} \\
& \varepsilon_{x y}=\frac{1}{2}\left(\frac{\partial u}{\partial y}+\frac{\partial v}{\partial x}\right)=-z \frac{\partial^{2} w}{\partial x^{2}}
\end{aligned}
$$

Considerando a lei de Hooke (2.5) temos:

$$
\begin{aligned}
& \sigma_{x x}=-\frac{E z}{1-v^{2}}\left(\frac{\partial^{2} w}{\partial x^{2}}+v \frac{\partial^{2} w}{\partial y^{2}}\right) \\
& \sigma_{y y}=-\frac{E z}{1-v^{2}}\left(\frac{\partial^{2} w}{\partial y^{2}}+v \frac{\partial^{2} w}{\partial x^{2}}\right) \\
& \sigma_{x y}=-2 G z \frac{\partial^{2} w}{\partial x \partial y}
\end{aligned}
$$

Vamos agora considerar o equilíbrio. As equações de equilíbrio para um corpo tridimensional são:

$$
\begin{aligned}
& \frac{\partial \sigma_{x x}}{\partial x}+\frac{\partial \sigma_{y x}}{\partial y}+\frac{\partial \sigma_{z x}}{\partial z}+X=0 \\
& \frac{\partial \sigma_{x y}}{\partial x}+\frac{\partial \sigma_{y y}}{\partial y}+\frac{\partial \sigma_{z y}}{\partial z}+Y=0 \\
& \frac{\partial \sigma_{x z}}{\partial x}+\frac{\partial \sigma_{y z}}{\partial y}+\frac{\partial \sigma_{z z}}{\partial z}+Z=0
\end{aligned}
$$


Agora, multiplicando a primeira equação (2.14) por zdz e integrando-a de -h/2 até h/2, obtemos:

$$
\begin{aligned}
& \int_{-h / 2}^{h / 2} \frac{\partial \sigma_{x x}}{\partial x} z d z+\int_{-h / 2}^{h / 2} \frac{\partial \sigma_{y x}}{\partial y} z d z+ \\
& +\int_{-h / 2}^{h / 2} \frac{\partial \sigma_{z x}}{\partial z} z d z+\int_{-h / 2}^{h / 2} X z d z=0
\end{aligned}
$$

Desde que as distribuições de tensão ao longo da espessura são agora conhecidas, podem ser calculados os valores dos momentos por unidade de comprimento, dados por:

$$
\begin{aligned}
& M_{x x}=\int_{-h / 2}^{h / 2} \sigma_{x x} z d z \\
& M_{y y}=\int_{-h / 2}^{h / 2} \sigma_{y y} z d z \\
& M_{x y}=\int_{-h / 2}^{h / 2} \sigma_{x y} z d z
\end{aligned}
$$

Podem também ser calculados os valores das forças cortantes por unidade de comprimento, como resultantes das integrais das tensões tangenciais ao longo da espessura:

$$
\begin{aligned}
& Q_{x}=\int_{-h / 2}^{h / 2} \sigma_{z x} d z \\
& Q_{y}=\int_{-h / 2}^{h / 2} \sigma_{z y} d z
\end{aligned}
$$

Os momentos externos aplicados por unidade de comprimento são expressos por: 


$$
\begin{aligned}
& m_{x}=\int_{-h / 2}^{h / 2} X z d z \\
& m_{y}=\int_{-h / 2}^{h / 2} Y z d z
\end{aligned}
$$

Na expressão (2.15), a ordem das operações de integração e diferenciação pode ser trocada nos primeiros dois termos, o terceiro termo pode ser calculado por integração por partes. Após isto, e substituindo os valores de (2.16), (2.17) e (2.18), fazendo o mesmo para a segunda equação de (2.14) vem:

$$
\begin{aligned}
& \frac{\partial M_{x x}}{\partial x}+\frac{\partial M_{x y}}{\partial y}-Q_{x}+m_{x}=0 \\
& \frac{\partial M_{y x}}{\partial x}+\frac{\partial M_{y y}}{\partial y}-Q_{y}+m_{y}=0
\end{aligned}
$$

Integrando-se agora a terceira equação (2.14) ao longo de $\mathrm{z}$ desde -h/2 até h/2 e procedendo-se analogamente ao citado acima temos:

$$
\frac{\partial Q_{x}}{\partial x}+\frac{\partial Q_{y}}{\partial y}+q=0
$$

onde q é a carga atuante resultante na direção z, dada por:

$$
q=\sigma_{z z}\left(\frac{h}{2}\right)-\sigma_{z z}\left(-\frac{h}{2}\right)+\int_{-h / 2}^{h / 2} Z d z
$$

Eliminando Qx e Qy na (2.19) por seus valores na (2.17) obtemos a equação de equilíbrio em momentos:

$$
\frac{\partial^{2} M_{x x}}{\partial x^{2}}+2 \frac{\partial^{2} M_{x y}}{\partial x \partial y}+\frac{\partial^{2} M_{y y}}{\partial y^{2}}=-q-\frac{\partial m_{x}}{d x}-\frac{\partial m_{y}}{\partial y}(2.22)
$$


Substituindo os valores das tensões de (2.13) em (2.16) obtemos as expressões momento-curvatura:

$$
\begin{aligned}
& M_{x x}=-D\left(\frac{\partial^{2} w}{\partial x^{2}}+v \frac{\partial^{2} w}{\partial y^{2}}\right) \\
& M_{y y}=-D\left(\frac{\partial^{2} w}{\partial y^{2}}+v \frac{\partial^{2} w}{\partial x^{2}}\right) \\
& M_{x y}=-D(1-v) \frac{\partial^{2} w}{\partial x \partial y}
\end{aligned}
$$

onde:

$$
D=\frac{E h^{3}}{12\left(1-v^{2}\right)}
$$

A expressão (2.24), chamada de rigidez à flexão da placa, transforma, numérica e dimensionalmente as curvaturas (ou suas combinações lineares) em momentos. A menos do fator $\left(1-v^{2}\right)$ trata-se do produto de rigidez $E J=\mathrm{bd}^{3} / 12$ de uma faixa de placa de largura unitária. O fator $\left(1-v^{2}\right)$ conduz, porém a D>EJ, ou seja, a placa é mais rígida que a viga, ou melhor, conforme MARTINELLI et al. (1986), sempre se deveria considerar o D, mesmo em vigas, quando se calcula com o EJ tem-se a impressão de rigidez algo menor, embora sendo a diferença pequena.

Para obter a equação constitutiva da cortante, a relação tensão-deformação não pode ser usada, pois, a deformação no plano médio é zero. Assim, recorre-se às condições de equilíbrio (2.19) e obtém-se:

$$
Q_{\alpha}=-D \frac{\partial}{\partial x_{\alpha}}\left(\frac{\partial^{2} w}{\partial x^{2}}+\frac{\partial^{2} w}{\partial y^{2}}\right)
$$


Derivando as relações momento-curvatura (2.23) e substituindo em (2.22) temse a equação fundamental da teoria linear de flexão de placas de espessura uniforme:

$$
\frac{\partial^{4} w}{\partial x^{4}}+2 \frac{\partial^{4} w}{\partial^{2} x \partial^{2} y}+\frac{\partial^{4} w}{\partial y^{4}}=\frac{1}{D}\left(q+\frac{\partial m_{x}}{\partial x}+\frac{\partial m_{y}}{\partial y}\right)
$$

ou, de forma compacta:

$$
\nabla^{4} w=\frac{1}{D}\left(q+\frac{\partial m_{x}}{d x}+\frac{\partial m_{y}}{d y}\right)
$$

A equação (2.26) mostra que a solução do problema de placas é reduzida à solução de equações biharmônicas com as adequadas condições de contorno.

Devido à equação diferencial ser de quarta ordem, apenas dois valores devem ser satisfeitos no problema de valores de contorno associados a cada ponto, ao invés de três valores, como seria o usual pelo caráter tridimensional do modelo. Isto ocorre devido à não consideração da deformação por cortante.

Foi demonstrado por KIRCHHOFF (1850) que a solução pode ser obtida agrupando-se as condições de contorno relativas à força cortante e ao momento na direção ns perpendicular ao contorno da placa, criando-se uma força cortante equivalente dada por:

$$
V_{n}=Q_{n}+\frac{\partial M_{n s}}{\partial s}
$$

A expressão (2.28) faz uso de um sistema auxiliar de coordenadas onde as coordenadas ns são posicionadas no contorno da placa, a coordenada s percorre o contorno no sentido anti-horário e a coordenada n, normal ao contorno é dirigida para o exterior da placa, $\mathbf{Q n}$ é a cortante na direção da normal e Mns é o momento volvente da borda considerada. 


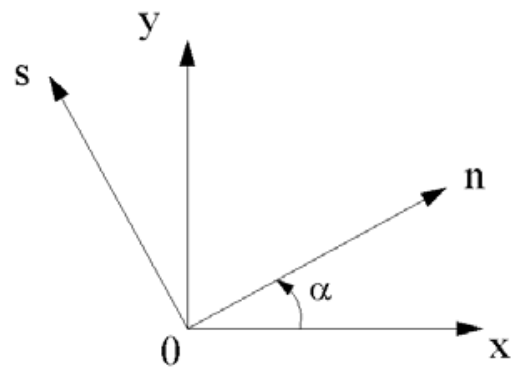

Figura 2.3 - Sistema de coordenadas ns

A transformação de coordenadas do sistema $(\mathrm{x}, \mathrm{y})$ para o sistema $(\mathrm{n}, \mathrm{s})$ é dada por $^{2}$ :

$$
\left\{\begin{array}{l}
x \\
y
\end{array}\right\}=\mathbf{T}\left\{\begin{array}{l}
n \\
s
\end{array}\right\}, \quad\left\{\begin{array}{l}
n \\
s
\end{array}\right\}=\mathbf{T}^{\mathbf{T}}\left\{\begin{array}{l}
x \\
y
\end{array}\right\}
$$

onde a matriz de transformação T é dada por:

$$
\mathbf{T}=\left[\begin{array}{cc}
\cos \alpha & -\operatorname{sen} \alpha \\
\operatorname{sen} \alpha & \cos \alpha
\end{array}\right]=\left[\begin{array}{cc}
n_{1} & t_{1} \\
n_{2} & t_{2}
\end{array}\right]
$$

onde a primeira coluna são os cossenos diretores do versor na direção $n$, enquanto que a segunda coluna são os cossenos diretores do versor na direção t.

A transformação do tensor de momentos do sistema (x,y) para o sistema $(\mathrm{n}, \mathrm{s})$ é dada por:

$$
\overline{\mathbf{M}}=\mathbf{T}^{\mathrm{T}} \mathbf{M} \mathbf{T}
$$

onde:

${ }^{2}$ As matrizes e vetores são indicadas em negrito. 


$$
\mathbf{M}=\left[\begin{array}{ll}
M_{x x} & M_{x y} \\
M_{y x} & M_{y y}
\end{array}\right], \quad \overline{\mathbf{M}}=\left[\begin{array}{ll}
M_{n n} & M_{n s} \\
M_{s n} & M_{s s}
\end{array}\right] \ldots(2.32)
$$

Considerando que Mxy = Myx, o tensor momento será representado com maior simplicidade através do vetor:

$$
\mathbf{M}=\left\{\begin{array}{l}
M_{x x} \\
M_{x y} \\
M_{y y}
\end{array}\right\}, \quad \overline{\mathbf{M}}=\left\{\begin{array}{l}
M_{n n} \\
M_{n s} \\
M_{s s}
\end{array}\right\}
$$

Utilizando o produto matricial para fazer a transformação das matrizes de momentos, agora transformadas em vetores, temos:

$$
\overline{\mathbf{M}}=\mathbf{C ~ M}
$$

onde a matriz C de transformação é dada por:
$\mathbf{C}=\left[\begin{array}{c}\cos ^{2} \alpha \\ -\cos \alpha \operatorname{sen} \alpha \\ \operatorname{sen}^{2} \alpha\end{array}\right.$
$2 \operatorname{sen} \alpha \cos \alpha$
$\operatorname{sen}^{2} \alpha$
$\cos ^{2} \alpha \operatorname{sen} \alpha$
$\operatorname{sen} \alpha \cos \alpha$
$-2 \operatorname{sen} \alpha \cos \alpha$
$\cos ^{2} \alpha$

Analogamente, para a transformação dos esforços cortantes do sistema $(\mathrm{x}, \mathrm{y})$ para o sistema $(n, s)$ temos:

$$
\overline{\mathbf{Q}}=\mathbf{T}^{\mathrm{T}} \mathbf{Q}
$$

onde: 


$$
\mathbf{Q}=\left\{\begin{array}{l}
Q_{x} \\
Q_{y}
\end{array}\right\}, \quad \overline{\mathbf{Q}}=\left\{\begin{array}{l}
Q_{n} \\
Q_{s}
\end{array}\right\}
$$

Um aspecto importante na teoria de Kirchhoff é a existência de forças no canto, que são introduzidas devido à variação dos momentos volventes na cortante distribuída, conforme (2.28). Estes termos correspondem às hipóteses simplificadoras assumidas pela teoria.

A Teoria de Kirchhoff recebeu muitas críticas e gerou energia para outros desenvolvimentos na teoria de placas, mas, conforme FUNG (1965), foi a mais importante descoberta na teoria de placas.

\subsection{TEORIA DE REISSNER}

A razão formal para a impossibilidade de satisfazer mais de duas condições pela Teoria Clássica tem sido a ordem da equação básica desta teoria; fisicamente esta razão está no fato que a distorção dos elementos da placa devida a forças cortantes foi negligenciada.

Este fato é equivalente a considerar o módulo de elasticidade transversal $G=\infty$; em algumas situações, procedendo desta maneira nós substituímos o material da placa, suposto isotrópico, por outro que não é. Atendendo à consideração de $G$, a placa não responde à rotação de algum momento aplicado à superfície da placa, se o vetor que representa o momento coincidir com a normal à esta superfície. Isto nos habilita a somar a variação $\partial \mathrm{Mns} / \partial \mathrm{s}$ devida a momentos volventes, com o efeito da força vertical Qn criando a força cortante equivalente e reduzindo o número de condições de contorno para duas.

A análise de placas elásticas é grandemente simplificada por esta redução. Por outro lado, considerando o já exposto, não podemos esperar completa concordância entre a distribuição de esforços teórica e a que realmente ocorre. 
O erro da Teoria Clássica para o cálculo de placas delgadas ocorre principalmente nas bordas e ao redor de furos com dimensões comparáveis à espessura da placa.

A correção inicial da teoria clássica com respeito ao efeito das deformações por força cortante é devido a Eric Reissner.

A Teoria de Reissner interpreta o problema das placas delgadas, com pequenos deslocamentos, considera as deformações devidas à força cortante, tem como hipóteses básicas:

- A espessura é pequena se comparada às outras dimensões;

- O material da placa é homogêneo, isotrópico e elástico-linear;

- Uma reta inicialmente normal ao plano médio, após a deformação da placa permanece reta, porém não mais normal ao plano médio, devido à consideração das deformações por força cortante;

- As componentes tangenciais são nulas nas faces da placa;

- A tensão normal à superfície externa das placas é dada por:

$$
\sigma_{33}= \pm \frac{q}{2} \text { para } x_{3}= \pm \frac{h}{2}
$$

OBSERVAÇÃO: Pode também ser admitida carga de um só lado da placa, ou seja, sem que se alterem os valores dos esforços a serem obtidos. Neste caso, teríamos:

$$
\sigma_{33}=0, \quad x_{3}=\frac{h}{2}, \quad \sigma_{33}=-q, \quad x_{3}=-\frac{h}{2}
$$

A Teoria de Reissner foi concebida a partir de uma distribuição de tensões assumida, a partir do qual foram escritas equações levando em consideração os efeitos da força cortante.

Coerente com a terceira hipótese é admitida variação linear para as tensões no plano da placa, ou seja:

$$
\sigma_{\alpha \beta}=\frac{12 M_{\alpha \beta} z}{h^{3}}
$$


Das duas primeiras equações (2.14), temos:

$$
\sigma_{\alpha 3}=\frac{3 Q_{\alpha}}{2 h}\left[1-\left(\frac{2 x_{3}}{h}\right)^{2}\right]
$$

Considerando a terceira equação (2.14), na ausência de forças de volume, vem:

$$
\sigma_{33,3}=-\sigma_{13,1}-\sigma_{23,2}
$$

Integrando a equação acima em relação à $\mathbf{x}_{\mathbf{3}}$ e impondo os valores da quinta hipótese temos:

$$
\sigma_{33}=\left\{\frac{1}{4}\left(\frac{2 x_{3}}{h}\right)\left[3-\left(\frac{2 x_{3}}{h}\right)^{2}\right]-\frac{1}{2}\right\} q
$$

A partir daí, Reissner recorreu ao método da energia para obter as equações diferenciais do problema, conforme já mostrado por RIBEIRO (1992) e SILVA (1996); utilizando o princípio de Reissner cujo funcional na ausência de forças de volume é dado por:

$$
\pi_{R}=-U^{*}-\int_{V} \sigma_{i j, j} u_{i} d V+\int_{\Gamma_{p}}\left(p_{i}-\bar{p}_{i}\right) u_{i} d s+\int_{\Gamma_{u}} p_{i} u_{i} d s
$$

onde:

$u_{i}$ são deslocamentos

$p$ são forças na superfície de contorno

$p$ são forças prescritas no contorno

$\Gamma_{p}$ é o trecho do contorno onde as forças são prescritas

$\Gamma_{u}$ é o trecho do contorno onde os deslocamentos são prescritos 
$U^{*}$ é a energia de deformação complementar, igual à energia de deformação para o caso admitido de material elástico linear.

$\sigma_{i j, j}$ são derivadas das tensões

onde as grandezas livres para variar são as tensões, os deslocamentos e as forças atuantes no contorno de deslocamentos prescritos.

Um outro método, que chega praticamente aos mesmos resultados, devido a E. Green é mostrado por TIMOSHENKO \& WOINOWSKY-KRIEGER (1959).

Na primeira versão do trabalho de Reissner, este foi contestado, pois escreveu a energia total como função das tensões assumidas e utilizou as relações constitutivas do espaço tridimensional, o que causa inconsistência entre deformações e tensões se todas as equações forem usadas. Assim, não seria possível empregar a equação:

$$
\frac{\partial w}{\partial x_{3}}=\frac{1}{E}\left[\sigma_{33}-v\left(\sigma_{11}+\sigma_{22}\right)\right]
$$

pois produziria diferenças no grau dos polinômios que representam $\sigma_{33}$ em relação aos adotados em (2.41).

Reissner publicou então uma segunda versão de sua teoria corrigindo-a definindo deslocamentos generalizados que, segundo ele, representam quantidades equivalentes, mas não idênticas às componentes de rotação da superfície média e que as deflexões w deveriam ser entendidas como um valor médio. Introduziu então as seguintes expressões adicionais:

$$
\begin{aligned}
& \int_{h} \sigma_{11} u_{1} d z=M_{11} \phi_{1} \\
& \int_{h} \sigma_{22} u_{2} d z=M_{22} \phi_{2} \\
& \int_{h} \sigma_{12} u_{1} d z=M_{12} \phi_{1}
\end{aligned}
$$




$$
\begin{aligned}
& \int_{h} \sigma_{13} u_{3} d z=Q_{1} w \\
& \int_{h} \sigma_{23} u_{3} d z=Q_{2} w
\end{aligned}
$$

Substituindo as equações (2.38), (2.39) e (2.41) em (2.43) obtemos:

$$
\begin{aligned}
& \phi_{\alpha}=\frac{12}{h^{3}} \int_{h} u_{\alpha} z d z \\
& w=\frac{3}{2 h} \int_{h}\left[1-\left(\frac{2 z}{h}\right)^{2}\right] w d z
\end{aligned}
$$

Os esforços podem então ser obtidos a partir dos deslocamentos:

$$
\begin{aligned}
& M_{11}=D\left(\phi_{1,1}+v \phi_{2,2}\right)+\frac{v}{(1-v) \lambda^{2}} q \\
& M_{22}=D\left(\phi_{2,2}+v \phi_{1,1}\right)+\frac{v}{(1-v) \lambda^{2}} q \\
& M_{12}=D\left(\phi_{1,2}+\phi_{2,1}\right) \\
& Q_{1}=D \frac{(1-v)}{2} \lambda^{2}\left(\phi_{1}+w_{, 1}\right) \\
& Q_{2}=D \frac{(1-v)}{2} \lambda^{2}\left(\phi_{2}+w_{, 2}\right)
\end{aligned}
$$

onde $\lambda^{2}=10 / h^{2}$ é uma constante característica da Teoria de Reissner. Escrevendo as equações (2.45) e (2.46) na forma indicial vem: 


$$
\begin{aligned}
& M_{\alpha \beta}=D \frac{(1-v)}{2}\left(\phi_{\alpha, \beta}+\phi_{\beta, \alpha}+\frac{2 v}{1-v} \phi_{\gamma, \gamma} \delta_{\alpha \beta}\right)+\frac{v q}{(1-v) \lambda^{2}} \delta_{\alpha \beta} \\
& Q_{\alpha}=D \frac{1-v}{2} \lambda^{2}\left(\phi_{\alpha}+w_{, \alpha}\right) \ldots \ldots \ldots \ldots \ldots \ldots \ldots \ldots \ldots \ldots \ldots \ldots \ldots \ldots \ldots \ldots \ldots \ldots \ldots \ldots \ldots \ldots \ldots \ldots
\end{aligned}
$$

Substituindo os valores de (2.45) e (2.46) nas equações de equilíbrio dadas em (2.19) e (2.20) obtém-se as equações de equilíbrio em termos de deslocamentos:

$$
\frac{\partial w}{\partial x_{1}}+\phi_{1}-\frac{h^{2}}{5(1-v)}\left[\begin{array}{l}
\frac{\partial^{2} \phi_{1}}{\partial x_{1}^{2}}+v \frac{\partial^{2} \phi_{2}}{\partial x_{1} \partial x_{2}} \\
+\frac{1-v}{2}\left(\frac{\partial^{2} \phi_{1}}{\partial x_{1}^{2}}+\frac{\partial^{2} \phi_{2}}{\partial x_{1} \partial x_{2}}\right)
\end{array}\right]-
$$

$\frac{6 v h(1+v)}{25 E(1-v)} \frac{\partial q}{\partial x_{2}}=0$

$$
\frac{\partial w}{\partial x_{2}}+\phi_{2}-\frac{h^{2}}{5(1-v)}\left[\begin{array}{l}
\frac{\partial^{2} \phi_{2}}{\partial x_{2}{ }^{2}}+v \frac{\partial^{2} \phi_{1}}{\partial x_{1} \partial x_{2}} \\
+\frac{1-v}{2}\left(\frac{\partial^{2} \phi_{2}}{\partial x_{2}{ }^{2}}+\frac{\partial^{2} \phi_{1}}{\partial x_{1} \partial x_{2}}\right)
\end{array}\right]-
$$

$\frac{6 v h(1+v)}{25 E(1-v)} \frac{\partial q}{\partial x_{1}}=0$

$$
\frac{\partial}{\partial x_{1}}\left(\frac{\partial w}{\partial x_{1}}+\phi_{1}\right)+\frac{\partial}{\partial x_{2}}\left(\frac{\partial w}{\partial x_{2}}+\phi_{2}\right)+\frac{24(1+v)}{10 E h} q=0
$$


As três equações de equilíbrio dadas em (2.19) e (2.20) combinadas com as cinco equações dadas em (2.45) e (2.46) formam um sistema de oito equações diferenciais parciais de primeira ordem de Reissner, onde as oito incógnitas são os três momentos, as duas cortantes e os deslocamentos, ou seja:

$$
M_{11}, M_{12}, M_{22}, Q_{1}, Q_{2}, \phi_{1}, \phi_{2}, w
$$

Considerando a equação diferencial de quarta ordem da Teoria Clássica, alguns autores fazem manipulação algébrica adotando hipótese de serem diferenciáveis as funções deslocamentos envolvidas e chegam a uma equação diferencial de Reissner em termos de deslocamentos:

$$
D \nabla^{4} w=q-\frac{(2-v)}{(1-v)} \frac{1}{\lambda^{2}} \nabla^{2} q
$$

\subsection{TEORIA DE MINDLIN}

A Teoria de Mindlin, percebendo os problemas apresentados pela Teoria de Reissner, buscou outra maneira de interpretar o problema das placas delgadas, com pequenos deslocamentos, considerando também as deformações devidas à força cortante.

Em lugar de adotar distribuição de tensões, Mindlin adotou uma distribuição de deformações conhecida, similarmente à Teoria Clássica. Como agora existem as deformações por força cortantes, as distorções não são mais nulas, Mindlin impôs nula a variação da distorção:

$$
\begin{aligned}
& \frac{\partial \gamma_{13}}{\partial x_{3}}=0 \\
& \frac{\partial \gamma_{23}}{\partial x_{3}}=0
\end{aligned}
$$


Ainda de forma similar à Teoria Clássica ele supõe nula a deformação na direção da espessura, desta forma temos:

$$
\varepsilon_{33}=0
$$

Integrando as equações (2.49) na espessura:

$$
\begin{aligned}
& \gamma_{13}=\frac{1}{G^{\prime}} \sigma_{13}=f_{1}\left(x_{1}, x_{2}\right) \\
& \gamma_{23}=\frac{1}{G^{\prime}} \sigma_{23}=f_{2}\left(x_{1}, x_{2}\right)
\end{aligned}
$$

Desta forma, existe valor não nulo para a distorção na superfície média, dada pelas funções f. O valor de G’ dado pela literatura clássica é dado por:

$$
G^{\prime}=\frac{\pi^{2}}{12} G
$$

Análise mais completa do valor de G’ é feita por PALERMO (2000).

Os deslocamentos podem então ser dados por:

$$
\begin{aligned}
& \frac{\partial u_{1}}{\partial x_{3}}=\alpha_{1}-\frac{\partial w}{\partial x_{1}} \\
& \frac{\partial u_{2}}{\partial x_{3}}=\alpha_{2}-\frac{\partial w}{\partial x_{2}}
\end{aligned}
$$

Definindo-se duas novas grandezas para o membro esquerdo das equações acima, chegamos a uma expressão para as deformações $\underline{\varepsilon}$ similar à da Teoria Clássica, ou seja: 


$$
\begin{aligned}
& \Psi_{1}=\alpha_{1}-\frac{\partial w}{\partial x_{1}} \\
& \Psi_{2}=\alpha_{2}-\frac{\partial w}{\partial x_{2}} \\
& \varepsilon_{11}=x_{3} \frac{\partial \Psi_{1}}{\partial x_{1}} \\
& \varepsilon_{22}=x_{3} \frac{\partial \Psi_{2}}{\partial x_{2}} \\
& \gamma_{12}=\left(\frac{\partial \Psi_{2}}{\partial x_{1}}+\frac{\partial \Psi_{1}}{\partial x_{2}}\right) \\
& \gamma_{13}=\frac{\partial w}{\partial x_{1}}+\Psi_{1} \\
& \gamma_{23}=\frac{\partial w}{\partial x_{2}}+\Psi_{2}
\end{aligned}
$$

Integrando-se as expressões (2.55) e (2.56) ao longo da espessura e considerando as expressões (2.16) e (2.17) temos:

$$
M_{11}=D\left(\frac{\partial \Psi_{1}}{\partial x_{1}}+v \frac{\partial \Psi_{2}}{\partial x_{2}}\right)
$$




$$
\begin{aligned}
& M_{22}=D\left(\frac{\partial \Psi_{1}}{\partial x_{1}} v+\frac{\partial \Psi_{2}}{\partial x_{2}}\right) \\
& M_{12}=D \frac{1-v}{2}\left(\frac{\partial \Psi_{2}}{\partial x_{1}}+\frac{\partial \Psi_{1}}{\partial x_{2}}\right) \\
& Q_{1}=G^{\prime} h\left(\frac{\partial w}{\partial x_{1}}+\Psi_{1}\right) \\
& Q_{2}=G^{\prime} h\left(\frac{\partial w}{\partial x_{2}}+\Psi_{2}\right)
\end{aligned}
$$

Utilizando a notação indicial para escrever as equações acima temos:

$$
\begin{aligned}
& M_{\alpha \beta}=D \frac{1-v}{2}\left(\Psi_{\alpha, \beta}+\Psi_{\beta, \alpha}+\frac{2 v}{1-v} \Psi_{\gamma, \gamma} \delta_{\alpha \beta}\right) \\
& Q_{\alpha}=D \frac{1-v}{2} \lambda^{2}\left(\Psi_{\alpha}+w_{, \alpha}\right) \ldots \ldots \ldots \ldots \ldots \ldots \ldots \ldots \ldots \ldots \ldots \ldots \ldots \ldots \ldots
\end{aligned}
$$

onde:

$$
\lambda^{2}=\frac{\pi^{2}}{h^{2}}
$$

A expressão acima é bastante similar à da constante característica de Reissner.

Escrevendo as equações de equilíbrio (2.19) e (2.20) e considerando os valores dos esforços das equações (2.59) e (2.60) obtém-se as equações de equilíbrio em termos de deslocamentos: 


$$
\frac{\partial w}{\partial x_{1}}+\Psi_{1}-\frac{2 h^{2}}{\pi^{2}(1-v)}\left[\begin{array}{l}
\frac{\partial^{2} \Psi_{1}}{\partial x_{1}^{2}}+v \frac{\partial^{2} \Psi_{2}}{\partial x_{1} \partial x_{2}}+ \\
\frac{1-v}{2}\left(\frac{\partial^{2} \Psi_{2}}{\partial x_{1} \partial x_{2}}+\frac{\partial^{2} \Psi_{1}}{\partial x_{2}^{2}}\right)
\end{array}\right]=0
$$

$$
\frac{\partial w}{\partial x_{2}}+\Psi_{2}-\frac{2 h^{2}}{\pi^{2}(1-v)}\left[\begin{array}{l}
\frac{\partial^{2} \Psi_{2}}{\partial x_{1}^{2}}+v \frac{\partial^{2} \Psi_{1}}{\partial x_{1} \partial x_{2}}+ \\
\frac{1-v}{2}\left(\frac{\partial^{2} \Psi_{1}}{\partial x_{1} \partial x_{2}}+\frac{\partial^{2} \Psi_{2}}{\partial x_{1}^{2}}\right)
\end{array}\right]=0
$$

$$
\frac{\partial}{\partial x_{1}}\left(\frac{\partial w}{\partial x_{1}}+\Psi_{1}\right)+\frac{\partial}{\partial x_{2}}\left(\frac{\partial w}{\partial x_{2}}+\Psi_{2}\right)+\frac{24(1+v)}{\pi^{2} E h} q=0
$$

Algumas observações podem aqui ser anotadas:

O valor do carregamento distribuído aparece apenas na equação de equilíbrio de cortantes, similarmente ao Método Clássico e diferente das equações de Reissner;

As equações apresentam similaridade de forma com as equações equivalentes de Reissner, aparentando serem similares às grandezas $\phi$ e $\psi$. Em verdade as funções $\psi$ de Mindlin são similares às de Timoshenko para a teoria clássica, enquanto que as funções $\phi$ de Reissner são definidas por ele próprio como "quantidades equivalentes, mas não idênticas às componentes da rotação na superfície média”.

A equação diferencial de Mindlin para as placas, em deslocamentos, é: 


$$
\nabla^{4} w=\frac{1}{D}\left(1-\frac{2}{1-v} \frac{1}{\lambda^{2}} \nabla^{2} q\right)
$$

\subsection{CAMPOS DE MOMENTOS INICIAIS}

Com a finalidade de analisar a ocorrência de ações devidas a campos de deformações iniciais como temperatura, etc. o tensor de deformações pode ser representado por:

$$
\varepsilon_{i j}=\varepsilon_{i j}^{e}+\varepsilon_{i j}^{0}
$$

onde:

$\varepsilon_{i j}^{0}$ é o campo das deformações iniciais e $\boldsymbol{\varepsilon}_{i j}^{e}$ é a componente elástica, obtida a partir do carregamento e das condições de contorno, supondo comportamento elástico do material.

A formulação a partir de deformações iniciais foi mostrada por VENTURINI (1983), aqui, entretanto será mostrada a formulação em termos de um campo de momentos iniciais, que possibilitará inclusive o uso de carregamentos incrementais, conforme já adotado por RIBEIRO (1992) e SILVA (1996).

As curvaturas iniciais podem ser relacionadas aos momentos iniciais por:

$$
M_{\alpha \beta}^{0}=C_{\alpha \beta \gamma \theta} \chi_{\gamma \theta}^{0}
$$

onde:

$C_{\alpha \beta \gamma \theta}$ é o tensor elástico de quarta ordem do material e $\chi_{\gamma \theta}^{0}$ são as componentes do campo de curvaturas iniciais.

Considerando as equações acima temos:

$$
M_{\alpha \beta}=M_{\alpha \beta}^{e}-M_{\alpha \beta}^{0}
$$


onde $M_{\alpha \beta}^{e}$ é a parcela do momento calculada elasticamente e $M_{\alpha \beta}$ é o momento verdadeiro considerando-se os momentos iniciais e o carregamento.

Considerando as expressões da Teoria de Reissner:

$$
\begin{aligned}
& M_{\alpha \beta}^{e}=C_{\alpha \beta \gamma \theta} \chi_{\gamma \theta}=C_{\alpha \beta \gamma \theta} \phi_{\gamma, \theta} \\
& M_{\alpha \beta}=M_{\alpha \beta}^{e}+\frac{v}{(1-v) \lambda^{2}} q \delta_{\alpha \beta}-M_{\alpha \beta}^{0}
\end{aligned}
$$

Analogamente para cortantes temos:

$$
\begin{aligned}
& Q_{3 \alpha}=C_{3 \alpha 3 \theta} \psi_{3 \theta} \\
& \psi_{3 \theta}=\phi_{\theta}+w_{, \theta}
\end{aligned}
$$

A figura abaixo ilustra as deformações por cisalhamento ocorridas na placa, onde a reta perpendicular à superfície média na posição indeformada, permanece reta após a deformação, mas não perpendicular à superfície média.

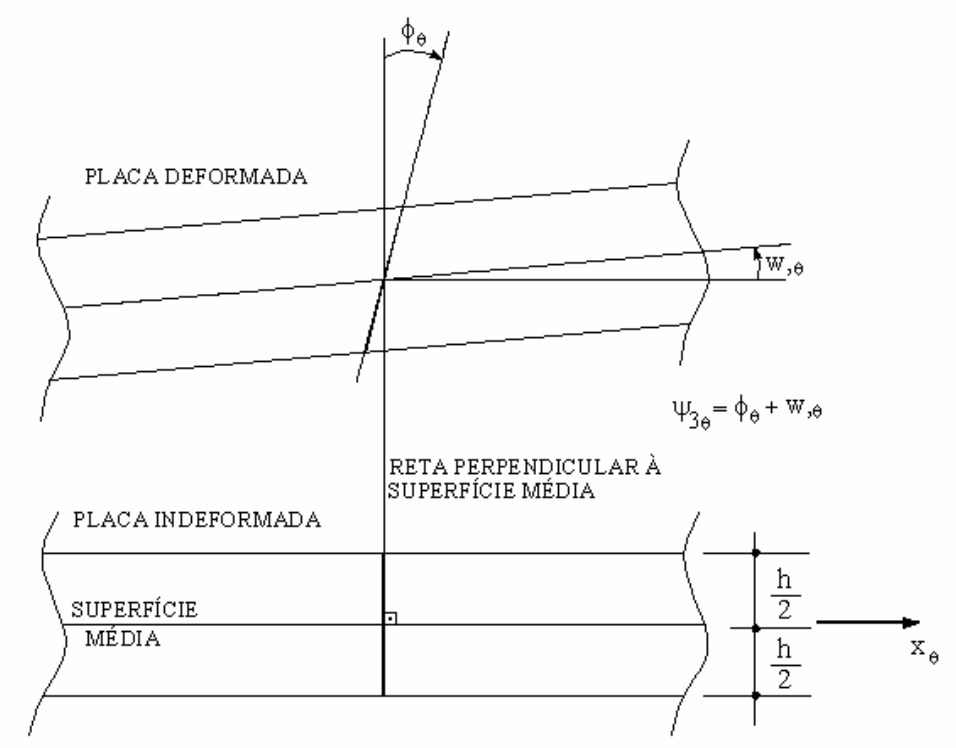

Figura 2.4 - Deformação por cisalhamento transversal 


\subsection{CONDIÇÕES DE CONTORNO}

Considerando-se a Teoria de Reissner, devem ser satisfeitas três condições físicas para cada ponto do contorno. Em placas, as condições usuais de contorno são:

Borda simplesmente apoiada, onde podem ser prescritas duas diferentes condições de contorno:

$$
\begin{aligned}
& w=0, \quad M_{n}=0, \quad \phi_{s}=0 \text { chamada de "hard condition", ou } \\
& w=0, \quad M_{n}=0, \quad M_{n s}=0 \text { chamada de "soft condition" }
\end{aligned}
$$

onde $\underline{\mathbf{n}}$ e $\underline{\mathbf{s}}$ são os eixos normal e tangencial ao contorno da placa. A condição “hard” é a usada na teoria clássica de Kirchhoff.

Borda engastada, onde as condições prescritas são:

$$
w=0, \quad \phi_{n}=0, \quad \phi_{s}=0
$$

Borda livre, com as seguintes condições de contorno:

$$
M_{n}=0, \quad M_{n s}=0, \quad Q_{n}=0
$$

\subsection{SOLUÇÕES FUNDAMENTAIS}

A utilização do Método dos Elementos de Contorno no estudo de placas traz a necessidade do estudo das chamadas soluções fundamentais. Fisicamente, solução fundamental é a resposta medida em um ponto t (ponto campo) de um domínio, geralmente infinito, causada pela aplicação de uma carga (ou binário) unitária em outro ponto s (source $=$ fonte) deste domínio. Guardam relação importante com as chamadas funções potencial, que medem a energia necessária para mover uma partícula de carga (ou massa) unitária colocada no ponto t, distando r do ponto s, até o infinito.

Matematicamente, a carga unitária é representada pela função denominada Delta de Dirac $\delta(s, t)$ que tem as seguintes propriedades: 


$$
\begin{aligned}
& \delta(s, t)=0, \quad s \text { não coincidente com } t \\
& \delta(s, t)=\infty, \quad s \equiv t \\
& \int_{\Omega} \phi(t) \delta(s, t) d \Omega=\phi(s) \ldots \ldots \ldots \ldots \ldots \ldots \ldots \ldots \ldots
\end{aligned}
$$

onde a função $\phi$ é definida no domínio $\Omega$.

$$
\int_{\Omega} \delta(s, t) d \Omega=1
$$

\subsubsection{SOLUÇÃO FUNDAMENTAL PARA A TEORIA DE KIRCHHOFF}

A solução fundamental, representada por $\mathrm{w}^{*}$ representa o deslocamento

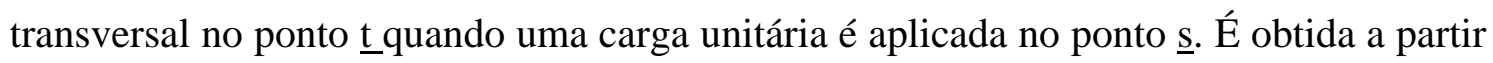
da equação das placas de Kirchhoff onde se substitui a carga distribuída pela função Delta de Dirac, ou seja:

$$
\nabla^{4} w=\delta(s, t) / D
$$

Considerando que a função é nula em todos os pontos distintos da fonte, temos em verdade que resolver a equação acima para o segundo membro nulo.

A solução foi mostrada por PAIVA (1987) escrevendo a equação (6.67) em coordenadas polares, onde temos:

$$
\nabla^{2} w=\left(\frac{\partial^{2}}{\partial r^{2}}+\frac{1}{r} \frac{\partial}{\partial r}+\frac{1}{r^{2}} \frac{\partial^{2}}{\partial \theta^{2}}\right) w
$$

tendo como resultado:

$$
w^{*}(s, t)=\frac{1}{8 \pi D} r^{2}\left(\ln r-\frac{1}{2}\right)
$$

conforme já obtido por DANSON (1979). 


\subsubsection{SOLUÇÃO FUNDAMENTAL PARA A TEORIA DE REISSNER}

A solução fundamental da Teoria de Reissner é obtida a partir das equações de equilíbrio em função dos deslocamentos generalizados $\mathrm{u}_{\mathrm{j}}$, ou seja:

$$
\Delta_{i j}^{*}\left(\frac{\partial}{\partial s}\right) u_{j}(s)=-b_{i}(s)
$$

Supondo um carregamento unitário dado pelo Delta de Dirac localizado no ponto fonte s vem:

$$
\Delta_{i j}^{*}=\left(\frac{\partial}{\partial t}\right) u_{k j}^{*}(s, t)=-\delta(s, t) \delta_{i k}
$$

onde $\delta_{i k}$ é o Delta de Kronecker.

A equação acima foi resolvida por WEEËN (1982) utilizando o método de Hörmander e vale:

$$
\begin{aligned}
& u_{\alpha \beta}^{*}=\frac{1}{8 \pi D(1-v)}\left\{\begin{array}{l}
\left\lfloor 8 B-(1-v)(2 \ln z-1) \delta_{\alpha \beta}\right\rfloor \\
-\left[8 A+2(1-v) r_{, \alpha} r_{, \beta}\right]
\end{array}\right\} \\
& u_{\alpha 3}^{*}=-u_{3 \alpha}^{*}=\frac{1}{8 \pi D}(2 \ln z-1) r r_{, \alpha} \\
& u_{33}^{*}=\frac{1}{8 \pi D(1-v) \lambda^{2}}\left[(1-v) z^{2}(\ln z-1)-8 \ln z\right]
\end{aligned}
$$

onde r equivale à distância entre s e t orientado "fugindo" da fonte s e: 


$$
\begin{aligned}
& r_{, \alpha}=\frac{\partial}{\partial x_{\alpha}(t)} r=\frac{r_{\alpha}}{r} \\
& r_{\alpha}=x_{\alpha}(t)-x_{\alpha}(s) \\
& z=\lambda r
\end{aligned}
$$

Os valores de A e B são obtidos das funções de Bessel modificadas de ordem inteira. Ao contrário dos trabalhos anteriores, aqui não foram estas calculadas através de expansões polinomiais, mas sim através das funções existentes no próprio FORTRAN, o que contribui para aumentar a precisão.

A função A(z) é contínua enquanto que a função B(z) apresenta singularidade do tipo $\ln (\mathrm{z})$. Suas expressões são dadas por:

$$
\begin{aligned}
& A(z)=K_{0}(z)+\frac{2}{z}\left[K_{1}(z)-\frac{1}{z}\right] \\
& B(z)=K_{0}(z)+\frac{1}{z}\left[K_{1}(z)-\frac{1}{z}\right]
\end{aligned}
$$

Considerando a forma que foi obtida por Weeën a solução fundamental para a teoria de Reissner, vê-se que a mesma poderia ser usada também para a teoria de Mindlin. Isto foi confirmado por VILMANN \& DASGUPTA (1992), onde foi obtida para Mindlin a mesma solução fundamental de Reissner, com exceção de se usar $\mathbf{r}$ e não $\mathbf{z}$ como argumento do logaritmo. 


\section{EQUAÇÕES INTEGRAIS DE PLACAS - TEORIA DE REISSNER}

\subsection{INTRODUÇÃO}

A análise de placas pelo Método dos Elementos de Contorno passa pela obtenção das equações integrais do problema. Estas equações podem ser obtidas usando-se o método dos resíduos ponderados, ou pelo Teorema de Green ou ainda pelo Teorema da Reciprocidade de Betti.

São usadas em sua dedução as equações diferenciais de equilíbrio das placas e as relações constitutivas características de cada teoria.

Despreza-se sempre, por ser usualmente pequeno, o trabalho associado às deformações na direção da espessura da placa, mesmo nas teorias que consideram a deformação por força cortante.

\subsection{EQUAÇÕES INTEGRAIS DE PLACAS}

Considera-se uma placa isótropa de espessura h, contorno qualquer $\Gamma$ e domínio $\Omega$ contida numa placa infinita de contorno $\Gamma^{*}$ e domínio $\Omega *$. O contorno $\Gamma$ da placa é dividido em dois contornos $\Gamma \mathbf{u}$ onde são prescritos deslocamentos generalizados no contorno e Гр onde são prescritas forças generalizadas no contorno, isto é:

$$
u_{k}=\bar{u}_{k} \text { em } \Gamma_{u}
$$




$$
\begin{aligned}
& p_{k}=\bar{p}_{k} \text { em } \Gamma_{p} \\
& \Gamma=\Gamma_{u} \cup \Gamma_{p}
\end{aligned}
$$

sendo u os deslocamentos e p as forças.

A placa em questão é submetida a dois carregamentos não simultâneos, o segundo indicado por um asterisco. Pelo Teorema de Betti vem:

$$
\int_{V} \sigma_{i j}^{*} \varepsilon_{i j} d V=\int_{V} \sigma_{i j} \varepsilon_{i j}^{*} d V
$$

\subsubsection{EQUAÇÃO INTEGRAL PARA DESLOCAMENTOS DE PONTOS DO INTERIOR DA PLACA}

Substituindo-se as relações deformação/deslocamento e tensão/deslocamento, integrando-se por partes, conforme já mostrado por RIBEIRO (1992) obtém-se a equação integral dos deslocamentos generalizados $\mathrm{u}_{\mathrm{i}}$ de um ponto s no interior da placa:

$$
\begin{aligned}
& u_{i}(s)=\int_{\Gamma}\left[u_{i k}^{*}(s, T) p_{k}(T)-p_{i k}^{*}(s, T) u_{k}(T)\right] d \Gamma(t)+ \\
& +\int_{\Omega} q(t)\left[u_{i 3}^{*}(s, t)-\frac{v}{(1-v) \lambda^{2}} u_{i \alpha, \alpha}^{*}(s, t)\right] d \Omega(t)+ \\
& +\int_{\Omega} u_{i \alpha, \beta}^{*}(s, t) M_{\alpha \beta}^{0}(t) d \Omega(t)+\int_{\Omega} u_{i \alpha}^{*}(s, t) m_{\alpha}(t) d \Omega(t)
\end{aligned}
$$

onde os pontos s e t pertencem ao domínio $\Omega$ e T pertence ao contorno $\Gamma$ da placa, $u_{k}$ e $p_{k}$ são os deslocamentos e forças generalizadas sobre o contorno. 
$u_{i k}^{*}(S, t)$ é o deslocamento generalizado no ponto $t$ na direção i correspondente a uma força generalizada unitária aplicada no ponto s na direção k. Convenção análoga é usada para as forças generalizadas.

As soluções fundamentais que serão citadas agora, para os diferentes tipos de carregamento aqui referidos, serão listadas no Apêndice A deste trabalho.

\subsubsection{EQUAÇÃO INTEGRAL PARA DESLOCAMENTOS DE PONTOS DO CONTORNO DA PLACA}

Para a solução do problema de placas através do MEC temos que escrever a equação (3.2) para pontos do contorno da placa.

Para evitar o problema de ocorrência de singularidades, uma das alternativas é admitir um acréscimo no domínio cujo contorno é circular, de raio arbitrário $\varepsilon$ e escrever a equação integral para o ponto, que agora é interno.

Admitindo-se que o contorno adicional é suave e que as soluções fundamentais satisfazem a condição de Hölder, o raio arbitrário é feito tender a zero no limite. Após as operações necessárias, considerando-se um ponto S situado no contorno, chega-se a:

$$
\begin{aligned}
& C_{i k}(S) u_{k}(S)=\int_{\Gamma}\left[u_{i k}^{*}(S, T) p_{k}(T)-p_{i k}^{*}(S, T) u_{k}(T)\right] d \Gamma(T) \\
& +\int_{\Omega_{q}} q(t)\left[u_{i 3}^{*}(S, t)-\frac{v}{(1-v) \lambda^{2}} u_{i \alpha, \alpha}^{*}(S, t)\right] d \Omega(t) \\
& +\int_{\Omega_{m}} u_{i \alpha}^{*}(S, t) m_{\alpha}(t) d \Omega(t)+\int_{\Omega} u_{i \alpha, \beta}^{*}(S, t) M_{\alpha \beta}^{0}(t) d \Omega(t)
\end{aligned}
$$

O coeficiente $C_{i k}$ procura generalizar a equação (3.3), valendo 1 para pontos no interior do domínio, 0 fora dele. Para pontos sobre o contorno que tem contorno suave (tangente contínua) o valor é $1 / 2$ e as integrais são entendidas no sentido do valor 
principal de Cauchy. Outros valores podem ser deduzidos para C se o contorno não for suave no ponto $S$.

A influência do campo de momentos iniciais nos valores dos deslocamentos dos pontos do contorno é dada pela integral

$$
I I_{i \alpha \beta}(s)=\int_{\Omega} u_{i \alpha, \beta}^{*}(S, t) M_{\alpha \beta}^{0}(t) d \Omega(t)
$$

\subsubsection{EQUAÇÃO INTEGRAL PARA ESFORÇOS NOS PONTOS DO DOMÍNIO DA PLACA}

O cálculo dos esforços nos pontos do domínio é de grande importância principalmente no caso de análise não linear. O uso de uma equação integral apropriada permite o cálculo direto dos mesmos, sem ter que calcular os esforços nos pontos internos e derivá-los numericamente como é feito no Método das Diferenças Finitas ou no Método dos Elementos Finitos.

Procedendo desta forma temos:

$$
\begin{aligned}
& M_{\alpha \beta}(s)=\int_{\Gamma} u_{\alpha \beta k}^{*}(s, T) p_{k}(T) d \Gamma(T)- \\
& \int_{\Gamma} p_{\alpha \beta k}^{*}(s, T) u_{k}(T) d \Gamma(T)+ \\
& \int_{\Omega_{q}} r_{\alpha \beta}^{*}(s, t) q(t) d \Omega_{q}(t)+\frac{v}{(1-v) \lambda^{2}} q \delta_{\alpha \beta}+ \\
& +\int_{\Omega_{m}} t_{\alpha \beta \gamma}^{*}(s, t) m_{\gamma}(t) d \Omega_{m}(t)+I_{\alpha \beta}(s) \ldots \ldots \ldots \ldots \ldots \ldots . . . . .(3.5) \\
& \text { O valor de } I_{\alpha \beta}(s) \text { se origina da derivação do termo referente aos momentos }
\end{aligned}
$$

iniciais da equação (3.3), ou seja: 


$$
\begin{gathered}
I_{\alpha \beta}(s)=\frac{D(1-v)}{2}\left\{\frac{\partial}{\partial x_{\beta}(s)} \int_{\Omega} u_{\alpha \gamma, \theta}^{*}(s, t) M_{\gamma \theta}^{0}(t) d \Omega(t)\right\}+ \\
\frac{D(1-v)}{2}\left\{\frac{\partial}{\partial x_{\alpha}(s)} \int_{\Omega}^{*} u_{\beta \gamma, \theta}^{*}(s, t) M_{\gamma \theta}^{0}(t)\right\}+ \\
D v \delta_{\alpha \beta}\left\{\frac{\partial}{\partial x_{\eta}(s)} \int_{\Omega} u_{\eta \eta, \theta}^{*}(t) M_{\gamma \theta}^{0}(t) d \Omega(t)\right\} \ldots . .(3.6)
\end{gathered}
$$

Nesta operação podem estar envolvidas singularidades do tipo 1/r, procedimento para resolvê-las foi apresentado por MIKHLIN (1962) e aplicado em diversos trabalhos.

No cálculo das forças cortantes temos:

$$
\begin{aligned}
& Q_{e \beta}(s)=\int_{\Gamma} u_{3 \beta k}^{*}(s, T) p_{k}(T) d \Gamma(T)- \\
& \int_{\Gamma} p_{3 \beta k}^{*}(s, t) u_{k}(T) d \Gamma(T)+ \\
& \int_{\Omega_{q}} r_{3 \beta}^{*}(s, t) q(t) d \Omega_{q}(t)+ \\
& \int_{\Omega_{m}} t_{3 \beta \gamma}^{*}(s, t) m_{\gamma}(t) d \Omega_{m}(t)+I_{3 \beta}(s) \\
& I_{3 \beta}(s)=\frac{D(1-v) \lambda^{2}}{2}\left[\int_{\Omega} u_{\beta \gamma, \theta}^{*}(s, t) M_{\gamma \theta}^{0}(t) d \Omega(t)\right]+ \\
& +\frac{D(1-v) \lambda^{2}}{2}\left[\int_{\Omega} u_{3 \gamma, \theta}^{*}(s, t) M_{\gamma \theta}^{0}(t) d \Omega(t)\right] \ldots \ldots \ldots \ldots \ldots \ldots . .(3.8)
\end{aligned}
$$




\subsubsection{CARGAS E MOMENTOS DISTRIBUÍDOS NO DOMÍNIO DA PLACA}

A integral da carga distribuída das equações (3.2) e (3.3) pode ser transformada numa integral sobre o contorno da região carregada, conforme mostrado por WEEËN (1982), utilizando-se o teorema da divergência quando temos:

$$
\begin{aligned}
& \int_{\Omega_{q}} q(t)\left[u_{i 3}^{*}(s, t)-\frac{v}{(1-v) \lambda^{2}} u_{i \alpha, \alpha}^{*}(s, t)\right] d \Omega(t)= \\
& q(t) \int_{\Gamma_{q}}\left[v_{i, \alpha}^{*}(s, t)-\frac{v}{(1-v) \lambda^{2}} u_{i \alpha}^{*}(s, t)\right] n_{\alpha} d \Gamma_{q}(t)
\end{aligned}
$$

onde a função $V_{i, \alpha}^{*}(S, t)$ deve satisfazer a relação:

$$
v_{i, \alpha \alpha}^{*}(s, t)=u_{i 3}^{*}(s, t)
$$

As expressões fundamentais que atendem à condição acima são dadas por:

$$
\begin{aligned}
V_{\alpha}^{*} & =\frac{1}{128 \pi D \lambda^{2}} r_{, \alpha} r z^{2}(4 \ln z-5) \\
V_{3}^{*} & =\frac{-z^{2}}{256 \pi D \lambda^{4}(1-v)}\left[64(\ln z-1)-z^{2}(1-v)(2 \ln z-3)\right]
\end{aligned}
$$

A influência da carga uniformemente distribuída q no cálculo dos esforços nos pontos internos representada na equação (3.5) pela integral:

$$
\int_{\Omega_{q}} r_{i \beta}^{*}(s, t) q(t) d \Omega(t) \text { foi transformada em integrais no contorno da área }
$$

carregada da placa dadas por: 


$$
\begin{aligned}
& \int_{\Gamma_{q}} w_{\alpha \beta}^{*}(s, t) q(t) d \Gamma_{q}(t) \text { para momentos e } \\
& \int_{\Gamma_{q}} w_{3 \beta}^{*}(s, t) q(t) d \Gamma_{q}(t) \text { para forças cortantes }
\end{aligned}
$$

Os valores das soluções fundamentais w dadas acima podem ser encontrados em diversos trabalhos anteriores como SILVA(1992) e BACARJI(2000).

A integral utilizada para a carga distribuída pode também ser utilizada para cargas concentradas, quando então temos, no caso das equações de deslocamentos:

$$
I(s)=P\left[u_{i 3}^{*}(s, t)-\frac{v}{(1-v) \lambda^{2}} u_{i \alpha, \alpha}^{*}(s, t)\right]
$$

A influência da carga concentrada P no cálculo dos esforços nos pontos internos é dada diretamente, sem integração por:

$$
I_{\alpha \beta}=P r_{\alpha \beta}^{*}
$$

no caso de momentos e

$$
I_{3 \beta}=P r_{3 \beta}^{*}
$$

no caso de força cortante.

Caso a carga distribuída atue sobre um subdomínio retangular estreito de largura b, considera-se uma distribuição de carga equivalente sobre a linha média do subdomínio carregado conduzindo a:

$$
d \Omega_{q}=b d \Gamma_{q}, \quad \bar{q}=b q
$$

A parcela das equações integrais referentes à esta carga pode então ser transformada numa integral de contorno de forma que: 


$$
\begin{aligned}
& I_{1}=\int_{\Omega_{q}} q(t)\left[u_{i 3}^{*}(s, t)-\frac{v}{(1-v) \lambda^{2}} u_{i \alpha, \alpha}^{*}(s, t)\right] d \Omega_{q}(t)= \\
& =\int_{\Gamma_{q}}\left[u_{i 3}^{*}(s, t)-\frac{v}{(1-v) \lambda^{2}} u_{i \alpha, \alpha}^{*}(s, t)\right] \bar{q}(t) d \Gamma_{q}(t)
\end{aligned}
$$

A expressão acima fornece a influência das cargas distribuídas em linha na equação integral dos deslocamentos.

A influência da carga distribuída em linha nas expressões dos esforços internos é dada por:

$$
I_{1}=\int_{\Omega_{q}} r_{i \alpha}^{*}(s, t) q(t) d \Omega_{q}(t)=\int_{\Gamma_{q}} r_{i \alpha}^{*}(s, t) \bar{q} d \Gamma_{q}(t)
$$

Caso o ponto fonte esteja sobre a linha de carga, ocorrem singularidades, conduzindo depois de resolvidas a:

$$
I_{1}=\int_{\Gamma_{q}} r_{\alpha \beta}^{*}(s, t) \bar{q}(t) d \Gamma_{q}(t)
$$

nos momentos e

$$
I_{1}=\frac{n_{\alpha}}{\pi} \bar{q}(s)+\int_{\Gamma_{q}} r_{3 \alpha}^{*}(s, t) \bar{q}(t) d \Gamma_{q}(t)
$$

nas cortantes, onde $\alpha$ é o ângulo entre o contorno da carga e o eixo $\mathbf{x}_{1}$.

Para os momentos distribuídos em linha nas equações (3.2) e (3.3) temos as expressões: 


$$
\begin{aligned}
& \overline{m_{\gamma}}(t)=b m_{\gamma}(t) \\
& I_{m}=\int_{\Omega_{m}} t_{i \beta \gamma}^{*}(s, t) m_{\gamma}(t) d \Omega_{m}(t)=\int_{\Gamma_{m}} t_{i \beta \gamma}^{*}(s, t) \bar{m}_{\gamma}(t) d \Gamma_{m}(t)
\end{aligned}
$$

A contribuição da distribuição dos momentos em linha, caso o ponto fonte $\mathrm{s}$ estiver situado sobre a mesma fica:

$$
I_{m}^{\prime}=I_{m}+\bar{m}_{\gamma}(s) I_{\alpha \beta \gamma}^{*}(s, t)
$$

A contribuição da distribuição de momentos em linha no cálculo das forças cortantes, para pontos situados sobre a linha é dada de maneira análoga à anterior por:

$$
\int_{\Gamma_{m}} t_{3 \beta \gamma}^{*}(s, t) \overline{m_{\gamma}}(t) d \Gamma_{m}(t)
$$




\section{O MÉTODO DOS ELEMENTOS DE CONTORNO APLICADO À ANÁLISE DE PLACAS}

\subsection{INTRODUÇÃO}

Aqui são mostrados os procedimentos seguidos para a utilização da Teoria de Reissner na solução de problemas de placas.

As equações dadas, são de difícil solução analítica, sendo que para a sua quase totalidade dos problemas práticos usuais, faz-se nelas operações algébricas que permitem transformá-las num sistema de equações lineares. Esta transformação é iniciada pela discretização do contorno da placa em elementos lineares, chamados elementos de contorno, onde os deslocamentos e forças de superfície são interpolados através de funções aproximadoras.

As integrais de domínio são calculadas dividindo-se o domínio em pequenas áreas (aqui estas áreas são triangulares) chamadas células onde os valores das variáveis são calculados também através de funções aproximadoras.

Em cada ponto de colocação do contorno são produzidas três equações correspondentes ao deslocamento transversal, rotação normal ao contorno e rotação tangencial ao contorno.

Resolve-se o sistema, a partir daí são calculados os valores dos deslocamentos e esforços nos pontos internos da placa. 


\subsection{DISCRETIZAÇÃO DAS EQUAÇÕES INTEGRAIS}

Inicialmente, o contorno da placa, $\Gamma$, é aproximado através de elementos de contorno que são segmentos de reta, dados por dois pontos extremos, situados sobre o contorno da placa, e um ponto intermediário, médio entre os dois extremos. Estes pontos são denominados nós. As coordenadas dos nós são dadas através de um sistema local de coordenadas homogêneas onde ao nó médio é atribuída a coordenada homogênea 0 (zero) e aos nós extremos as coordenadas homogêneas +1 e -1 , com variação linear.

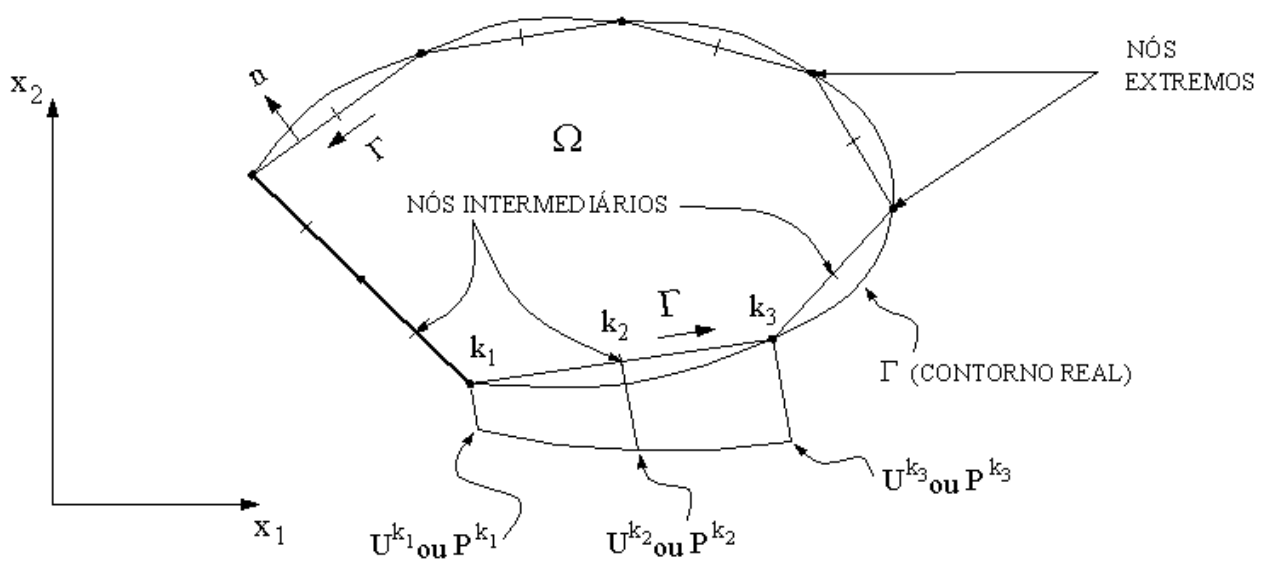

Figura 4.1 - Elementos do contorno discretizado

Para as coordenadas de um ponto P situado sobre o elemento, temos:

$$
\mathbf{x}^{P}=\boldsymbol{\psi}^{T}(\xi) \mathbf{x}^{(N)}
$$




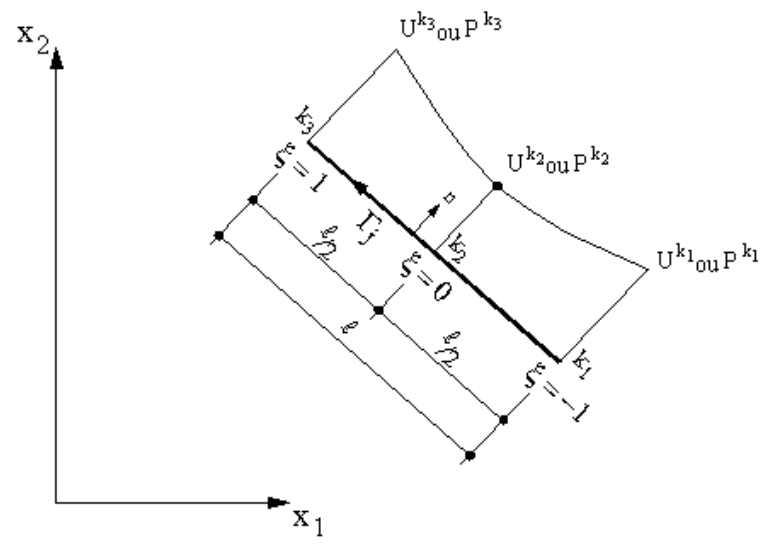

Figura 4.2 - Elemento do contorno e coordenadas homogêneas Valem seguintes relações:

$\mathbf{x}^{p}=\left\{\begin{array}{l}x_{1}^{P} \\ x_{2}^{P}\end{array}\right\}, \quad \mathbf{x}^{(N)}=\left\{\begin{array}{c}x_{1}^{k 1} \\ x_{1}^{k 3} \\ x_{2}^{k 1} \\ x_{2}^{k 3}\end{array}\right\}$

$\mathbf{u}^{k 1}=\left\{\begin{array}{l}u_{1}^{k 1} \\ u_{2}^{k 1} \\ u_{3}^{k 1}\end{array}\right\}, \quad \mathbf{p}^{k 1}=\left\{\begin{array}{l}p_{1}^{k 1} \\ p_{2}^{k 1} \\ p_{3}^{k 1}\end{array}\right\}$

$\boldsymbol{\psi}^{T}(\xi)=\left[\begin{array}{cc}\boldsymbol{\varphi}^{T}(\xi) & \mathbf{0} \\ \mathbf{0} & \boldsymbol{\varphi}^{T}(\xi)\end{array}\right]$ 


$$
\boldsymbol{\varphi}=\left\{\begin{array}{l}
\phi_{1} \\
\phi_{2}
\end{array}\right\}=\left\{\begin{array}{l}
(1-\xi) / 2 \\
(1+\xi) / 2
\end{array}\right\}
$$

Os valores das forças e deslocamentos são, por sua vez, aproximados por funções interpoladoras do segundo grau, em função agora dos três nós do elemento, conforme figura (4.2) acima. Para um ponto P situado no elemento temos:

$$
\mathbf{u}^{P}=\boldsymbol{\psi}_{0}^{T}(\xi) \mathbf{u}^{(N)}, \quad \mathbf{p}^{P}=\boldsymbol{\psi}_{0}^{T}(\xi) \mathbf{p}^{(N)}
$$

$$
\boldsymbol{\psi}_{0}^{T}(\xi)=\left[\begin{array}{ccccccccc}
\psi_{1} & 0 & 0 & \psi_{2} & 0 & 0 & \psi_{3} & 0 & 0 \\
0 & \psi_{1} & 0 & 0 & \psi_{2} & 0 & 0 & \psi_{3} & 0 \\
0 & 0 & \psi_{1} & 0 & 0 & \psi_{2} & 0 & 0 & \psi_{3}
\end{array}\right]
$$

$$
\begin{aligned}
& \psi_{1}=\xi(1-\xi) / 2 \\
& \psi_{2}=(1-\xi)(1+\xi) \\
& \psi_{3}=\xi(1+\xi) / 2
\end{aligned}
$$

$$
\mathbf{u}^{(N)}=\left\{\begin{array}{l}
\mathbf{u}^{k 1} \\
\mathbf{u}^{k 2} \\
\mathbf{u}^{k 3}
\end{array}\right\}, \quad \mathbf{p}^{(N)}=\left\{\begin{array}{l}
\mathbf{p}^{k 1} \\
\mathbf{p}^{k 2} \\
\mathbf{p}^{k 3}
\end{array}\right\}
$$

$$
\mathbf{u}^{k}=\left\{\begin{array}{l}
u_{1}^{k} \\
u_{2}^{k} \\
u_{3}^{k}
\end{array}\right\}=\left\{\begin{array}{l}
\phi_{n}^{k} \\
\phi_{s}^{k} \\
w^{k}
\end{array}\right\}, \quad \mathbf{p}^{k}=\left\{\begin{array}{c}
p_{1}^{k} \\
p_{2}^{k} \\
p_{3}^{k}
\end{array}\right\}=\left\{\begin{array}{c}
M_{n}^{k} \\
M_{s}^{k} \\
Q_{n}^{k}
\end{array}\right\}
$$


onde os valores significam:

$\phi_{n}, \quad \phi_{s}$ rotações nos planos normal $\mathbf{u}_{3} \mathbf{n}$ e tangencial $\mathbf{u}_{3} \mathbf{s}$, respectivamente, para pontos do contorno;

W - deslocamento transversal na direção $\mathbf{u}_{\mathbf{3}}$;

$M_{n}, \quad M_{n s}$ momentos nos planos $\mathbf{u}_{3} \mathbf{n}$ e $\mathbf{u}_{3} \mathbf{s}$, respectivamente, para pontos do contorno;

$Q_{n}$ força cortante na direção $\mathbf{u}_{3}$, para pontos do contorno.

\subsection{DESLOCAMENTOS NO CONTORNO DA PLACA}

Considerando a discretização do contorno, bem como a mudança de variável acima descritas, a equação (3.3) que descreve o cálculo dos deslocamentos em pontos do contorno da placa fica:

$$
\begin{aligned}
& C_{i k}(S) u_{k}(S)=\sum_{k=1}^{N E} \int_{-1}^{1}|J| u_{i k}^{*} \psi_{0 k}^{T} d \xi \mathbf{p}^{(N)}- \\
& \sum_{k=1}^{N E} \int_{-1}^{1}|J| p_{i k}^{*} \psi_{0 k}^{T} d \xi \mathbf{u}^{(N)}+\int_{\Omega_{q}} q(t)\left[u_{i 3}^{*}(S, t)-\right. \\
& \left.\frac{v}{(1-v) \lambda^{2}} u_{i \alpha, \alpha}^{*}(S, t)\right] d \Omega(t)+\int_{\Omega} u_{i \alpha, \beta}^{*}(S, t) M_{\alpha \beta}^{0}(t) d \Omega(t)+ \\
& \int_{\Omega_{m}} u_{i \alpha}^{*}(S, t) m_{\alpha}(t) d \Omega(t)
\end{aligned}
$$


onde $|J|=\frac{d \Gamma}{d \xi}=\frac{l_{j}}{2}$ é o jacobiano da transformação.

A partir daí são calculadas numericamente as matrizes $\mathrm{G}$ e H:

$$
\begin{aligned}
& g_{i k}^{n}(S)=\int_{-1}^{1}|J| u_{i k}^{*}(S, t) \psi_{n}(t) d \xi(t) \\
& h_{i k}^{n}(S)=\int_{-1}^{1}|J| p_{i k}^{*}(S, t) \psi_{n}(t) d \xi(t)
\end{aligned}
$$

onde:

$u_{i k}^{*}, \quad P_{i k}^{*}$ são as soluções fundamentais generalizadas para deslocamentos e forças de superfície;

$n \quad$ é o nó do elemento onde é medida a resposta ao carregamento unitário aplicado. Se $n=1$, o ponto t coincide com o ponto $k_{1}$. Quando $n=1$ ou $n=3$, o ponto t é nó de extremidade e pertence a dois elementos distintos, ambos são computados na matriz.

$S$ é o nó singular, ou seja, o ponto onde se aplica a carga unitária ou o ponto onde se escrevem as equações.

$\boldsymbol{i}$ é a equação que está sendo escrita, se $\mathrm{i}=1$ temos a equação de $\phi_{\mathbf{n}}$, se $\mathrm{i}=2$ temos a equação de $\phi_{\mathrm{s}}$ se $\mathrm{i}=3$ temos a equação de $\mathrm{w}$.

$k$ indica o tipo da resposta em $\mathrm{T}$. Se $\mathrm{k}=1$, a resposta corresponde à primeira linha dos vetores u ou p, ou seja, $\mathbf{M}_{\mathbf{n}}$ ou $\phi_{\mathbf{n}}$.

Gerando a equação (4.11) para os três pontos nodais de todos os NECP (número de elementos de contorno da placa) elementos, obtemos um sistema com $3 *$ NNCP (número de nós de contorno da placa) equações algébricas, que é dado por:

$$
\mathbf{C U}+\hat{\mathbf{H}} \mathbf{U}=\mathbf{G P}+\mathbf{B}+\mathbf{E M}^{\mathbf{0}}
$$

onde as outras matrizes e vetores representam: 
U, $\quad \mathbf{P}$ são os vetores de deslocamentos e forças de superfície referentes aos nós do contorno;

B é o vetor que dá a influência do carregamento de domínio nos valores dos deslocamentos e forças de superfície nos nós do contorno;

$\mathbf{E}$ é a matriz de influência do campo de momentos iniciais $\mathbf{M}^{\mathbf{0}}$ sobre os valores dos deslocamentos e forças de superfície nos nós do contorno, sua determinação é dada adiante.

Como a matriz $\mathbf{C}$ depende apenas da geometria do contorno em cada ponto considerado, podemos incorporá-la à matriz $\mathbf{H}$ obtendo:

\section{$\mathbf{H U}=\mathbf{G P}+\mathbf{B}+\mathbf{E M}^{0}$}

Se os pontos fonte forem colocados sobre o contorno da placa, teremos a ocorrência de algumas singularidades nas integrais acima, que podem ser evitadas colocando-se os pontos fonte externamente ao contorno, um ponto fora para cada ponto do contorno, a uma distância deste dada por:

$$
d=a l_{m}
$$

onde $\underline{\mathbf{a}}$ é um coeficiente positivo e $\underline{\mathbf{l}}_{\mathbf{m}}$ é o comprimento médio dos elementos adjacentes ao ponto considerado, ou o próprio comprimento do elemento quando o ponto coincide com o nó intermediário $\mathbf{k}_{\mathbf{2}}$. Neste caso, temos a vantagem adicional que para pontos externos ao contorno, a matriz $\mathbf{C}$ é nula. Este foi o procedimento adotado inicialmente por VENTURINI e aqui seguido.

Quanto menor o valor de d, melhores serão os resultados. As integrais são calculadas numericamente usando-se pontos de Gauss, aumentando-se o número de pontos quando d diminui aproximando-se do contorno. Neste trabalho as integrais foram feitas com 12 pontos de integração. Para valores de d muito pequenos, os resultados pioram, mesmo aumentando-se os pontos de integração. A solução nestes casos é a adoção de sub-elementos obtidos da subdivisão do elemento em outros menores. 
Nos cantos foram adotados nós duplos, para resolver o problema da descontinuidade da normal ao contorno, bem como à mudança das condições de contorno, pertencendo o canto a dois lados diferentes. Os nós duplos aqui empregados possuem as mesmas coordenadas, são situados um “antes” do nó e o outro “depois” do nó e assim tem valores nodais independentes.

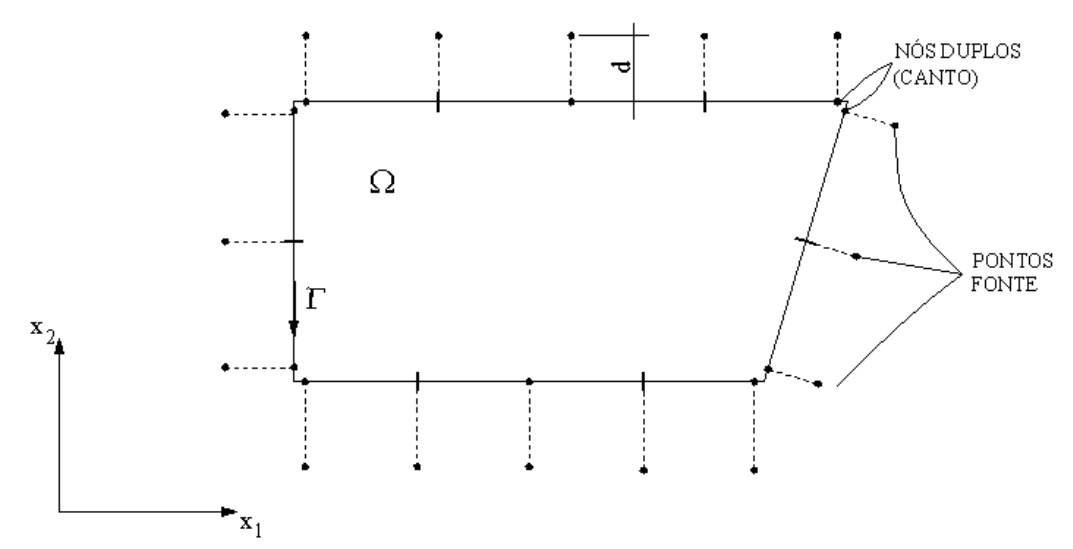

Figura 4.3 - Colocação dos pontos fonte fora do domínio

\subsection{DESLOCAMENTOS EM PONTOS INTERNOS}

Procedendo-se analogamente e discretizando-se a equação (3.2) obtemos a equação que nos permite calcular os deslocamentos nos pontos internos onde haja interesse, a partir dos deslocamentos dos pontos do contorno, já calculados.

$$
\overline{\mathbf{U}}=\overline{\mathbf{G}} \mathbf{P}-\overline{\mathbf{H}} \mathbf{U}+\overline{\mathbf{B}}+\overline{\mathbf{E}} \mathbf{M}^{0}
$$

onde os vetores e matrizes $\mathrm{U}, \mathrm{G}, \mathrm{H}, \mathrm{B}, \mathrm{E}$ com o traço superior tem significado análogo aos anteriormente citados após a expressão (4.13), referindo-se agora apenas a pontos internos. Nota-se aqui uma das vantagens do MEC sobre o MEF, pois pode ser calculado o deslocamento de qualquer ponto interno sem necessidade de interpolação.

Os coeficientes das matrizes $\mathbf{G}$ e $\mathbf{H}$ da equação acima não apresentam singularidades pois os pontos onde se calculam os deslocamentos são internos e as 
variáveis utilizadas referem-se ao contorno. Já com os termos do vetor B acima a integração deverá ser feita analiticamente quando o ponto interno para o qual se escrevem as equações coincidir com a carga concentrada ou com as linha de cargas ou de momentos.

Para o caso de uma carga concentrada $\mathbf{P}$ coincidir com o ponto de integração a expressão que mede sua contribuição no vetor B será dada por:

$$
\lim _{r \rightarrow 0} P\left[u_{i 3}^{*}-\frac{v}{(1-v) \lambda^{2}} u_{i \alpha, \alpha}^{*}\right]=0
$$

Para o caso de cargas distribuídas em linha o problema será tratado adiante.

\subsection{ESFORÇOS EM PONTOS INTERNOS AO DOMÍNIO}

São calculados fazendo-se a discretização das equações (3.5) para momentos e (3.7) para cortantes, chegando-se a:

$$
\begin{aligned}
& \mathbf{M}=\mathbf{G}^{\prime} \mathbf{P}-\mathbf{H}^{\prime} \mathbf{U}+\mathbf{B}^{\prime}+\left(\mathbf{E}^{\prime}-\mathbf{I}\right) \mathbf{M}^{\mathbf{0}} \\
& \mathbf{Q}=\mathbf{G}^{\prime \prime} \mathbf{P}-\mathbf{H}^{\prime \prime} \mathbf{U}+\mathbf{B}^{\prime \prime}+\mathbf{E}^{\prime \prime} \mathbf{M}^{\mathbf{0}}
\end{aligned}
$$

onde:

$\mathbf{P}, \quad \mathbf{U}$ são os vetores dos valores nodais das forças de superfície e dos deslocamentos;

$\mathbf{B}^{\prime}, \mathbf{B}^{\prime \prime}$ são os vetores que determinam a influência de todo o carregamento de domínio, exceto os momentos iniciais, sobre os momentos e forças cortantes nos pontos internos;

I é a matriz identidade;

$\mathbf{E}^{\prime}, \quad \mathbf{E}^{\prime \prime}$ são as matrizes de influência dos momentos iniciais sobre os momentos e cortantes nos pontos internos. 
Com convenientes transformações, a primeira equação (4.18) pode ser reescrita com vistas à análise não linear e, conforme SILVA(1996) como:

$$
\mathbf{M}^{e}=\mathbf{N}+\mathbf{S M}^{\mathbf{0}}
$$

onde:

$\mathbf{S}$ representa a influência dos momentos iniciais nos momentos dos pontos internos e de contorno e $\mathbf{N}$ representa a resposta elástica, sem considerar os momentos iniciais

\subsection{INFLUÊNCIAS DO CAMPO DE MOMENTOS INICIAIS}

\subsubsection{INTRODUÇÃO}

A consideração do campo de momentos iniciais atuando no domínio da placa é de fundamental importância, sendo usada em problemas de temperatura, retração e também com muita relevância na solução de problemas não-lineares.

A formulação do Método dos Elementos de Contorno para placas de Reissner e Mindlin foi proposta por WEEËN (1982) e BARCELLOS \& SILVA (1987) respectivamente. Recentemente, estas formulações tem sido discutidas em muitos trabalhos, em alguns dos quais se trata da visão unificada do MEC sobre estas teorias e também sobre a teoria de Kirchhoff, hipóteses estas propostas separadamente por WESTPHAL (2001) e PALERMO (2000), (2003).

Como estas formulações trabalham com formulações de grande complexidade, é conveniente que as integrais ao longo dos elementos de contorno sejam avaliadas precisamente. Estudos tratando desta matéria foram apresentados em muitos trabalhos como os de: EL-ZAFRANY (1995), RASHED et al. (1998) e MARCZAK \& CREUS (2002). Estes trabalhos são todos relacionados à precisão de se avaliar as integrais de contorno.

Neste trabalho, foi tentada uma nova maneira de melhorar a precisão dos efeitos do campo de momentos iniciais, com vistas à sua aplicação em análise não linear. 


\subsubsection{INFLUÊNCIA DOS MOMENTOS INICIAIS NOS DESLOCAMENTOS}

A influência do campo de momentos iniciais no cálculo dos deslocamentos é dada por:

$$
I_{i}(S)=\int_{\Omega} u_{i \alpha, \beta}^{*}(S, t) M_{\alpha \beta}^{0}(t) d \Omega(t)
$$

que pode ser dividida em:

$$
\begin{aligned}
& I_{\alpha \beta}(S)=\int_{\Omega} u_{i \alpha, \beta}^{*}(S, t) M_{\alpha \beta}^{0}(t) d \Omega(t), \quad \alpha=1,2 \\
& I_{3 \beta}(S)=\int_{\Omega} u_{3 \alpha, \beta}(S, t) M_{\alpha \beta}^{0}(t) d \Omega(t)
\end{aligned}
$$

De acordo com PALERMO (2000), a solução fundamental do problema de placas, modelos de Kirchhoff, Reissner e Mindlin é dada por:

Devido à carga unitária na direção ortogonal à superfície da placa:

$$
\begin{aligned}
& w^{*}=\frac{1}{\pi D}\left[\frac{1}{8} r^{2}(\ln \lambda r-1)-\frac{1}{(1-v) \lambda^{2}} \ln \lambda r\right] \\
& \phi_{\alpha}=-\frac{\partial}{\partial x_{\alpha}} \frac{1}{8 \pi D} r^{2}(\ln \lambda r-1)
\end{aligned}
$$

Devido a um binário unitário na direção $\mathbf{u}_{\alpha}$ temos $^{3}$ :

$u_{i \alpha}^{*}=-w_{, i \alpha}+\delta_{i \alpha} h_{, \xi \xi}-h_{, i \alpha}$

para $\mathrm{i}=1,2 \mathrm{e}$

$u_{3 \alpha}^{*}=-w_{, \alpha}$

${ }^{3}$ Alguns índices foram modificados para maior clareza. 
onde:

$$
\begin{aligned}
& w_{, i \alpha}=\frac{1}{8 \pi D} \frac{\partial}{\partial x_{i}} \frac{\partial}{\partial x_{\alpha}}\left[r^{2}(\ln \lambda r-1)\right] \\
& h_{, \alpha \beta}=\frac{1}{\pi D(1-v) \lambda^{2}} \frac{\partial}{\partial x_{\alpha}} \frac{\partial}{\partial x_{\beta}}\left[\ln (\lambda r)+K_{0}(\lambda r)\right] \\
& w_{, \alpha}=\frac{1}{8 \pi D} \frac{\partial}{\partial x_{\alpha}}\left[r^{2}(\ln (\lambda r)-1)\right]
\end{aligned}
$$

onde $\mathrm{K}_{0}$ é a função de Bessel modificada.

É interessante trabalhar na integral dada em (4.18), para reduzir sua singularidade evitando, entretanto, obter funções de Bessel de ordem mais elevada. Com esta idéia, podemos escrever, integrando por partes:

$$
\int_{\Omega} u_{i \alpha, \beta}^{*} M_{\alpha \beta}^{0} d \Omega=\int_{\Gamma} u_{i \alpha}^{*} \eta_{\beta} M_{\alpha \beta}^{0} d \Gamma-\int_{\Omega} u_{i \alpha}^{*} M_{\alpha \beta, \beta}^{0} d \Omega
$$

Considerando agora $\mathbf{i = 1 , 2}$ e substituindo as expressões de (4.26) pelas dadas acima vem:

$$
\begin{aligned}
& \int_{\Omega} u_{i \alpha, \beta}^{*} M_{\alpha \beta}^{0} d \Omega=\int_{\Gamma} u_{i \alpha}^{*} \eta_{\beta} M_{\alpha \beta}^{0} d \Gamma+ \\
& \int_{\Omega}\left(w_{, i \alpha}+\delta_{i \alpha} h_{, \xi \xi}+h_{, i \alpha}\right) M_{\alpha \beta, \beta}^{0}
\end{aligned}
$$

Aqui, o domínio da placa foi dividido em células triangulares, considerando-se linear a variação dos momentos iniciais sobre as mesmas. 

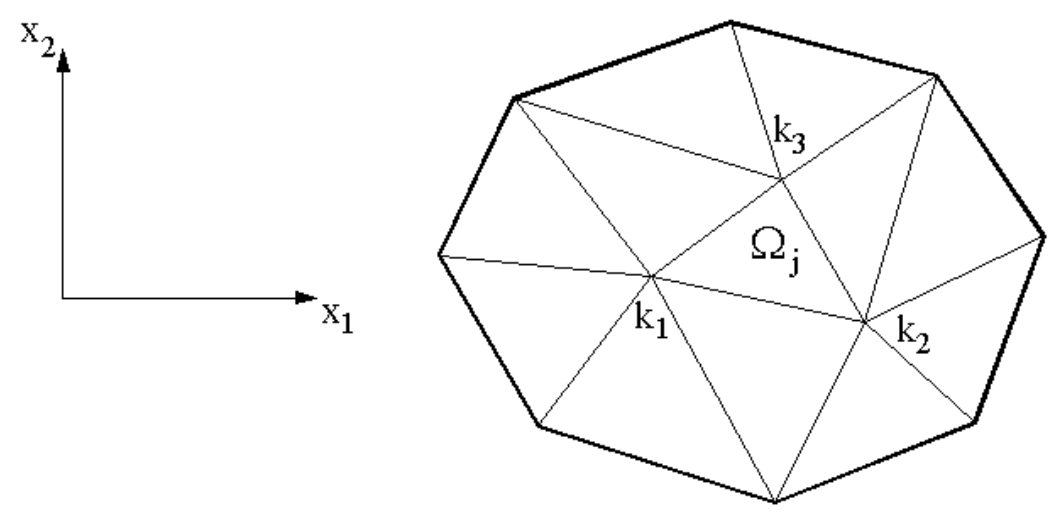

Figura 4.4 - Divisão do domínio da placa

Desenvolvendo-se por partes a segunda integral da equação (4.26) e levando em conta que, pela hipótese de variação linear de momentos nas células as segundas derivadas dos momentos são nulas quando calculadas nas células temos:

$$
\begin{aligned}
& I_{\alpha \beta}(S)=\int_{\Gamma} u_{i \alpha}^{*} \eta_{\beta} M_{\alpha \beta}^{0} d \Gamma+ \\
& \int_{\Gamma}\left(w_{, i} \eta_{\alpha}+h_{, i} \eta_{\alpha}+\delta_{i \alpha} h_{, \xi} \eta_{\xi}\right) M_{\alpha \beta, \beta}^{*} d \Gamma
\end{aligned}
$$

Deve ser notado que as integrais com densidade $M_{\alpha \beta}^{0}$ são calculadas no contorno externo da placa apenas e só as integrais com densidade $M_{\alpha \beta, \beta}^{0}$ devem ser calculadas ao longo do contorno das células se a continuidade da derivada do momento não é assumida.

A integral de domínio devida ao campo de momentos iniciais foi, portanto transformada para o contorno ou para sub-domínios do contorno, onde não há singularidades fortes a serem avaliadas.

Então, técnicas simples de integração numérica podem ser adotadas para calcular precisamente os efeitos dos momentos iniciais.

De forma similar, a integral referente à cortante fica: 


$$
\begin{aligned}
& I_{3 \beta}(S)=\int_{\Omega} u_{3 \alpha, \beta}^{*} M_{\alpha \beta}^{0} d \Omega= \\
& \int_{\Gamma} u_{3 \alpha}^{*} \eta_{\beta} M_{\alpha \beta}^{0} d \Gamma-\int_{\Omega} u_{3 \alpha}^{*} M_{\alpha \beta, \beta}^{0} d \Omega= \\
& \int_{\Gamma} u_{3 \alpha}^{*} \eta_{\beta} M_{\alpha \beta}^{0} d \Gamma+\int_{\Gamma} w \eta_{a} M_{\alpha \beta, \beta}^{0} d \Gamma
\end{aligned}
$$

\subsubsection{INFLUÊNCIA DOS MOMENTOS INICIAIS NOS ESFORÇOS}

Similarmente, da representação dos deslocamentos podemos obter as curvaturas e a partir daí as equações de momentos e forças cortantes para os pontos internos.

As integrais ao longo do contorno podem ser bem calculadas usando esquemas analíticos ou numéricos, conforme RASHED et al. (1998) e EL-ZAFRANY et al. (1995).

Aqui, estas integrais que calculam a influência dos momentos iniciais são dadas nas equações (3.6) no caso dos momentos fletor e torsor e (3.8) no caso das forças cortantes.

Da equação (3.6) vem:

$$
\begin{gathered}
I_{\alpha \beta}(s)=\frac{D(1-v)}{2}\left\{\frac{\partial}{\partial x_{\beta}(s)} \int_{\Omega} u_{\alpha \gamma, \theta}^{*}(s, t) M_{\gamma \theta}^{0}(t) d \Omega(t)\right\}+ \\
\frac{D(1-v)}{2}\left\{\frac{\partial}{\partial x_{\alpha}(s)} \int_{\Omega} u_{\beta \gamma, \theta}^{*}(s, t) M_{\gamma \theta}^{0}(t)\right\}+ \\
D v \delta_{\alpha \beta}\left\{\frac{\partial}{\partial x_{\eta}(s)} \int_{\Omega} u_{\eta \eta, \theta}^{*}(t) M_{\gamma \theta}^{0}(t) d \Omega(t)\right\}
\end{gathered}
$$

Considerando apenas a primeira integral acima temos: 


$$
\begin{aligned}
& V_{\alpha}=\int_{\Omega} u_{\alpha \gamma, \theta}^{*} M_{\gamma \theta}^{0} d \Omega= \\
& \int_{\Gamma} u_{\alpha \gamma}^{*} \eta_{\theta} M_{\gamma \theta}^{0} d \Gamma-\int_{\Omega} u_{\alpha \gamma}^{*} M_{\gamma \theta, \theta}^{0} d \Omega
\end{aligned}
$$

Calcula-se agora a derivada da expressão acima, que é possível ser realizada sem maiores preocupações, pois as integrais não contêm singularidades.

$$
\begin{aligned}
& \frac{\partial V_{\alpha}}{\partial x_{\beta}}=\int_{\Gamma} u_{\alpha \gamma, \beta}^{*} \eta_{\theta} M_{\gamma \theta}^{0} d \Gamma-\int_{\Omega} u_{\alpha \gamma, \beta}^{*} M_{\gamma \theta, \theta}^{0} d \Omega= \\
& \int_{\Gamma} u_{\alpha \gamma, \beta}^{*} \eta_{\theta} M_{\gamma \theta}^{0} d \Gamma-\int_{\Gamma} u_{\alpha \gamma}^{*} \eta_{\beta} M_{\gamma \theta, \theta}^{0}
\end{aligned}
$$

A passagem final foi obtida por integração por partes da segunda integral e considerando nula a segunda derivada do momento nas células. Procedendo analogamente para as outras integrais temos:

$$
\begin{aligned}
& I_{\alpha \beta}=\frac{D(1-v)}{2}\left[\int_{\Gamma}\left(u_{\alpha \gamma, \beta}^{*}+u_{\beta \gamma, \alpha}^{*}+\frac{2 v}{(1-v)} \delta_{\alpha \beta} u_{\eta \gamma, \eta}^{*}\right) \eta_{\theta} M_{\gamma \theta}^{0} d \Gamma\right]- \\
& \frac{D(1-v)}{2}\left[\int_{\Gamma}\left(u_{\alpha \gamma}^{*} \eta_{\beta}+u_{\beta \gamma}^{*} \eta_{\alpha}+\frac{2 v}{1-v} \delta_{\alpha \beta} u_{\eta \gamma}^{*} \eta_{\eta}\right) M_{\gamma \theta, \theta}^{0} d \Gamma\right]
\end{aligned}
$$

A integral que mostra a influência do campo de momentos iniciais nas forças cortantes é obtida de forma análoga chegando-se a: 


$$
I_{3 \beta}=\frac{D(1-v) \lambda^{2}}{2}\left[\begin{array}{l}
\int_{\Gamma}\left(u_{\beta \gamma}^{*} \eta_{\theta}-u_{3 \gamma, \beta}^{*}\right) \eta_{\theta} M_{\gamma \theta}^{0} d \Gamma+ \\
\int_{\Gamma}\left(w_{, \beta}+h_{, \beta}\right) \eta_{\gamma} M_{\gamma \theta, \theta}^{0} d \Gamma- \\
\int_{\Gamma}\left(\delta_{\beta \gamma} h_{, \xi} \eta_{\xi}-u_{3 \gamma}^{*} \eta_{\beta}\right) M_{\gamma \theta, \theta}^{0} d \Gamma
\end{array}\right]
$$

Novamente, as integrais acima podem ser calculadas analítica ou numericamente nos contornos das sub-regiões (células) sem necessitarem de qualquer esquema especial.

\subsubsection{DETALHES DAS CÉLULAS EMPREGADAS}

Cada célula é definida por seus três nós $\mathbf{k}_{1}, \mathbf{k}_{\mathbf{2}}$ e $\mathbf{k}_{3}$ nos quais são conhecidos os valores dos momentos iniciais com suas componentes.

O esquema da divisão, bem como os valores adotados para uma célula genérica são dados nas figuras abaixo. Para cada célula é estabelecido um sistema de coordenadas homogêneas para facilitar a integração numérica. 

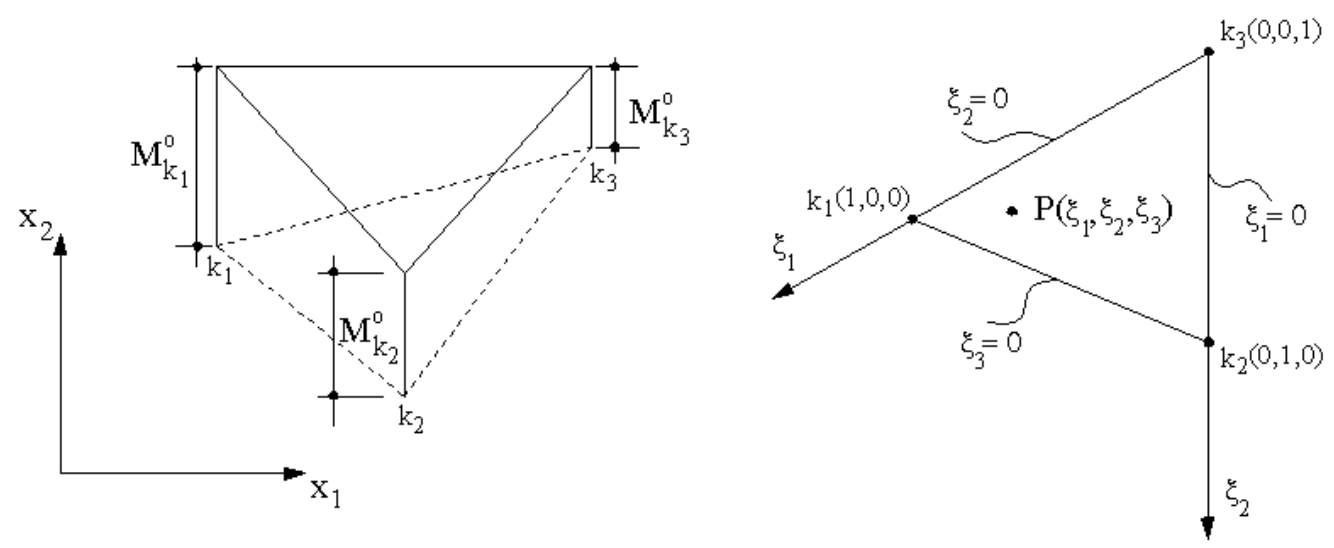

Figura 4.5 - Célula do domínio

Valem as seguintes relações:

$$
\begin{aligned}
& \mathbf{X}^{P}=\left\{\begin{array}{l}
X_{1} \\
X_{2}
\end{array}\right\}^{P}=\boldsymbol{\varphi}^{T} \mathbf{x}^{(N)}(4.35) \\
& \boldsymbol{\varphi}^{T}=\left[\begin{array}{llllll}
\xi_{1} & 0 & \xi_{2} & 0 & \xi_{3} & 0 \\
0 & \xi_{1} & 0 & \xi_{2} & 0 & \xi_{3}
\end{array}\right] \\
& \mathbf{x}^{(N)^{T}}=\left\{X_{1}^{k 1} X_{2}^{k 1} X_{1}^{k 2} X_{2}^{k 2} X_{1}^{k 3} X_{2}^{k 3}\right\} \\
& x_{i}^{P}=\xi_{1}^{P} x_{i}^{k 1}+\xi_{2}^{P} x_{i}^{k 2}+\xi_{3}^{P} x_{i}^{k 3}
\end{aligned}
$$

Podemos relacionar as coordenadas homogêneas do ponto $\mathrm{P}$ com suas coordenadas cartesianas através de: 


$$
\begin{aligned}
& \xi_{\alpha}^{P}=\frac{1}{2 A}\left(2 A_{0}^{\alpha}+b^{\alpha} x_{1}^{P}+a^{\alpha} x_{2}^{P}\right) \ldots \\
& a^{\alpha}=x_{1}^{k}-x_{1}^{j}, \quad b^{\alpha}=x_{2}^{j}-x_{2}^{k} \\
& 2 A_{0}^{\alpha}=x_{1}^{j} x_{2}^{k}-x_{1}^{k} x_{2}^{j} \\
& A=\left(b^{1} a^{2}-b^{2} a^{1}\right) / 2 \\
& \alpha=1,2,3 \quad j=2,3,1 \quad k=3,1,2
\end{aligned}
$$

O campo de momentos iniciais sobre a célula é aproximado em função dos valores nodais dos três vértices:

$$
\begin{aligned}
& \mathbf{M}^{0^{P}}=\left\{\begin{array}{l}
M_{1}^{0} \\
M_{2}^{0} \\
M_{3}^{0}
\end{array}\right\}=\boldsymbol{\psi}^{T} \mathbf{M}^{0^{(N)}}(4.40) \\
& \boldsymbol{\psi}^{T}=\left\{\begin{array}{ccccccccc}
\xi_{1} & 0 & 0 & \xi_{2} & 0 & 0 & \xi_{3} & 0 & 0 \\
0 & \xi_{1} & 0 & 0 & \xi_{2} & 0 & 0 & \xi_{3} & 0 \\
0 & 0 & \xi_{1} & 0 & 0 & \xi_{2} & 0 & 0 & \xi_{3}
\end{array}\right\} \\
& \mathbf{M}^{0^{(N)^{T}}}=\left\{\mathbf{M}^{0^{k 1}} \mathbf{M}^{0^{k 2}} \mathbf{M}^{k^{k 3}}\right\} \\
& \mathbf{M}^{0^{k i}}=\left\{M_{1}^{0^{k i}} M_{2}^{0^{k i}} M_{3}^{0^{k i}}\right\}=\left\{M_{x_{1} x_{1}}^{0^{k i}} M_{x_{1} x_{2}}^{0^{k i}} M_{x_{2} x_{2}}^{0^{k i}}\right\}
\end{aligned}
$$




\section{PLACAS COM ENRIJECEDORES - ACOPLAMENTO MEC/MEF}

\subsection{INTRODUÇÃO}

Em sua forma original, a análise de placas pelo Método dos Elementos de Contorno leva a um sistema de equações lineares cujas incógnitas são os valores dos deslocamentos e forças de superfície nos pontos de contorno. Este sistema não permite a análise de placas com vinculações internas, como pilares e vigas internas, usuais na prática.

Neste trabalho a equação (4.12) será modificada e acrescida de outras para analisar a presença de pontos ou linhas internas onde os deslocamentos ou esforços serão prescritos ou calculados, permitindo a análise das placas citadas acima. Esta modificação introduzirá novas incógnitas que por sua vez exigirão novas equações.

PAIVA (1987) apresentou formulação para análise de placas contínuas e lajes cogumelo supondo inicialmente que apenas os deslocamentos verticais dos pontos da interface laje-pilar estão impedidos, desprezando a resistência à flexão dos pilares. Posteriormente, no mesmo trabalho, desenvolveu formulação geral para análise da associação da placa com estrutura genérica. Particularizou as expressões da formulação geral para a análise da associação placa-pilar, considerando a rigidez do pilar à compressão e flexão. Analisou também a associação placa-grelha.

SILVA(1996) baseou-se nos trabalhos de PAIVA e estendeu a formulação para apoios em linhas, sendo o enrijecimento produzido pela estrutura formada pelas barras foi obtido pelo Método dos Elementos Finitos. 
BOTA(2003) na análise de chapas, introduziu na combinação das equações uma técnica de regularização baseada no método dos mínimos quadrados, onde o número de equações inicialmente existente é superior ao número de incógnitas.

Neste trabalho serão consideradas inicialmente as placas apoiadas sobre pilares. A seguir são apresentadas placas com carregamentos em linha que podem representar carregamentos prescritos ou reações nas interfaces placa-viga.

\subsection{EQUAÇÃO INTEGRAL PARA PLACAS COM VÍNCULOS INTERNOS AO DOMÍNIO}

A equação (4.12) quando modificada, pode incluir a influência de cargas distribuídas em linhas ou sub-regiões podendo ser interpretadas como carregamentos prescritos, conforme já visto, ou reações das interfaces placa-pilar e placa-viga, respectivamente.

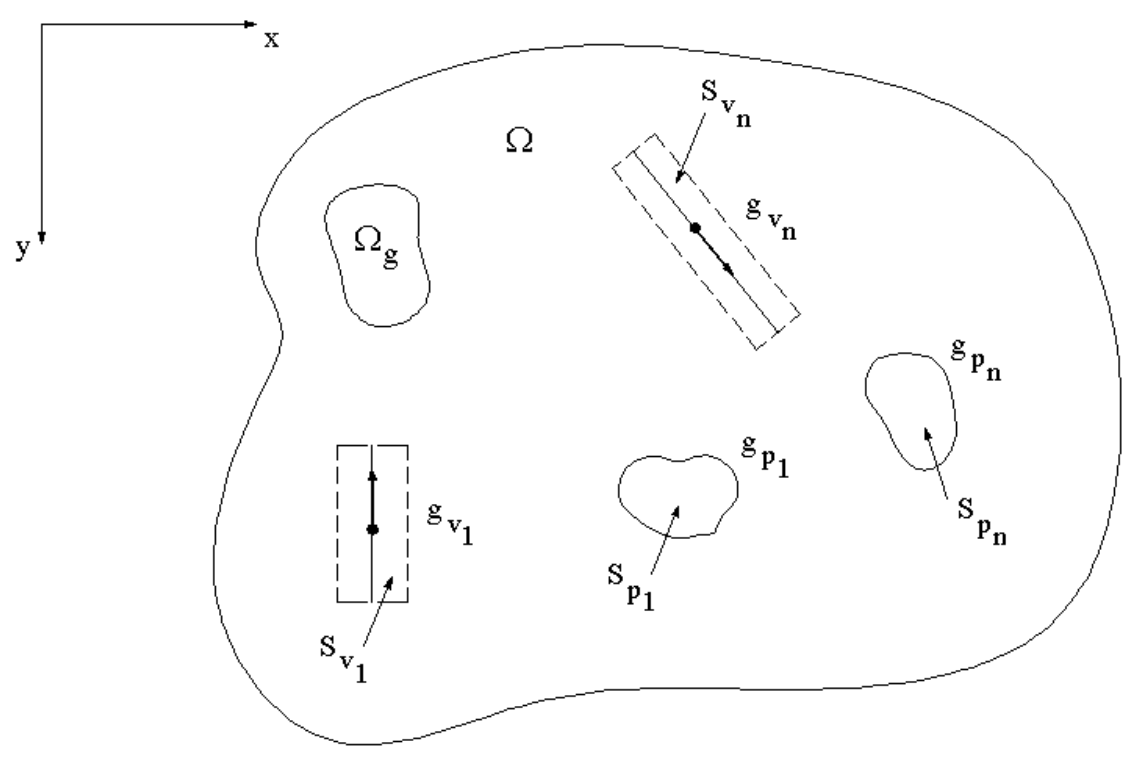

Figura 5.1 - Placa com linhas de carga e sub-regiões carregadas 
A equação que calcula os deslocamentos para um ponto qualquer do contorno fica:

$$
\begin{aligned}
& C_{i k}(S) u_{k}(S)=\int_{\Gamma}\left[u_{i k}^{*}(S, T) p_{k}(T)-p_{i k}^{*}(S, T) u_{k}(T)\right] d \Gamma(T) \\
& +\int_{\Omega_{q}} q(t)\left[u_{i 3}^{*}(S, t)-\frac{v}{(1-v) \lambda^{2}} u_{i \alpha, \alpha}^{*}(S, t)\right] d \Omega(t) \\
& +\int_{\Omega} u_{i \alpha, \beta}^{*}(S, t) M_{\alpha \beta}^{0}(t) d \Omega(t)
\end{aligned}
$$

onde nas áreas $\Omega_{q}$ devem agora ser incluídas as áreas referentes aos pilares e vigas internos ao contorno, conforme figura acima.

Procedimento análogo deve ser feito para as equações de esforços internos. Considerando a equação na sua forma matricial, fica:

$$
\mathbf{H U}=\mathbf{G P}+\mathbf{B}+\mathbf{S p}+\mathbf{E M}^{\mathbf{0}}
$$

onde temos que o vetor $\mathbf{B}$ armazena a influência de todo o carregamento externo aplicado à placa, exceto os momentos iniciais, carregamento este que pode estar atuando em áreas internas, pontos internos ou linhas internas.

Este carregamento, no caso de interfaces placa-pilar e placa-viga pode ser interpretado como um vetor de reações incógnitas $\mathbf{p}$, sendo que a matriz $\mathbf{S}$ representa a influência destes termos nos deslocamentos do contorno. 


\subsection{APOIOS PONTUAIS}

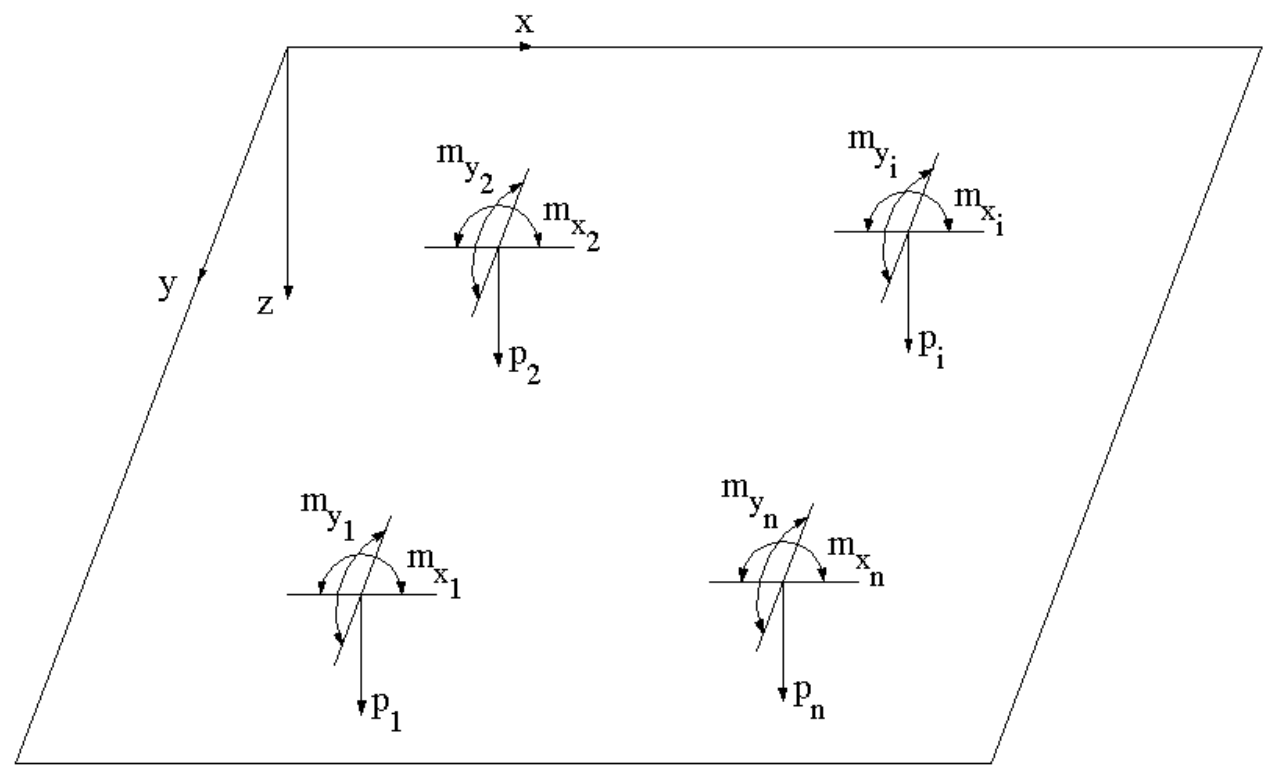

Figura 5.2 - Associação placa - pilares

Considerando uma placa com NAP apoios internos pontuais, as matrizes tem dimensões: $\mathbf{S}(3 N N C P, N A P)$ e $\mathbf{p}(\mathrm{NAP})$, onde:

$$
\begin{aligned}
& S_{\alpha \beta}=u_{\alpha \beta}^{*} \\
& S_{3 \alpha}=u_{3 \alpha}^{*} \\
& S_{i 3}=u_{i 3}^{*}+\frac{v}{(1-v) \lambda^{2}} u_{i \alpha, \alpha}^{*}
\end{aligned}
$$

As soluções fundamentais podem ser encontradas no Apêndice A.

Para cada apoio interno (pilar) temos 3 reações no vetor $\mathbf{p}$ que representam os momentos e a reação vertical na interface placa-pilar.

$$
\mathbf{p}^{\mathrm{T}}=\left[\begin{array}{lll}
\mathbf{R}_{\mathrm{Mx}} & \mathbf{R}_{\mathrm{My}} & \mathbf{R}_{\mathrm{w}}
\end{array}\right]
$$


A equação (5.1) representa um sistema com 3NNCP incógnitas às quais foram acrescidas agora 3NAP reações desconhecidas. As novas equações que se fazem necessárias, são obtidas escrevendo-se as 3NAP equações integrais referentes aos deslocamentos dos NAP pontos internos:

$$
\overline{\mathbf{U}}=\overline{\mathbf{G}} \mathbf{P}-\overline{\mathbf{H}} \mathbf{U}+\overline{\mathbf{B}}+\overline{\mathbf{S}} \mathbf{p}+\overline{\mathbf{E}} \mathbf{M}^{\mathbf{0}}
$$

No caso de lajes cogumelo, supondo que os “j” pilares tenham rigidez axial infinita e rigidezes à flexão nulas, serão nulos os deslocamentos $\mathbf{w}_{\mathbf{j}}$ e as reações $\mathbf{M x}_{\mathbf{j}}$ e $\mathbf{M y}_{\mathbf{j}}$. Reescrevendo as equações (5.1) e (5.3) agora formando um sistema onde as incógnitas são os deslocamentos $\mathbf{U}$ no contorno e as reações verticais $\mathbf{R}_{\mathbf{w}}$ nos pilares temos:

$$
\begin{aligned}
& {\left[\begin{array}{l}
\mathbf{H}-\mathbf{S} \\
\overline{\mathbf{H}}_{\mathrm{i}, 3 \mathbf{j}}-\overline{\mathbf{S}}_{\mathrm{i}, 3 \mathbf{j}}
\end{array}\right]\left\{\begin{array}{l}
\mathbf{U} \\
\mathbf{R}_{\mathrm{w}}
\end{array}\right\}=\left[\begin{array}{lr}
\mathbf{G} & \mathbf{0} \\
\overline{\mathbf{G}}_{\mathrm{i}, 3 \mathbf{j}} & -\mathbf{I}
\end{array}\right]\left\{\begin{array}{l}
\mathbf{P} \\
\mathbf{W}
\end{array}\right\}} \\
& +\left\{\begin{array}{l}
\mathbf{B} \\
\overline{\mathbf{B}}
\end{array}\right\}+\left\{\begin{array}{l}
\mathbf{E} \\
\overline{\mathbf{E}}
\end{array}\right\} \mathbf{M}^{\mathbf{0}}
\end{aligned}
$$

\subsection{APOIOS EM ÁREAS DISCRETAS}

Neste caso a área da interface placa-pilar é uma área que admite-se permanecer plana após a deformação ou seja, temos uma variação linear de tensões, que equivale a uma carga distribuída linearmente na superfície de contato.

Este assunto já foi estudado de diferentes formas por PAIVA(1987), SILVA (1996) e BACARJI (2000).

O sistema de referência adotado $\mathbf{x}_{1} \mathbf{x}_{2}$ tem os eixos paralelos aos eixos principais de inércia do pilar. Caso tal não ocorra será necessário incluir matriz de transformação do mesmo tipo da matriz A, usado quando houver vigas (ver equação (5.22) adiante). 

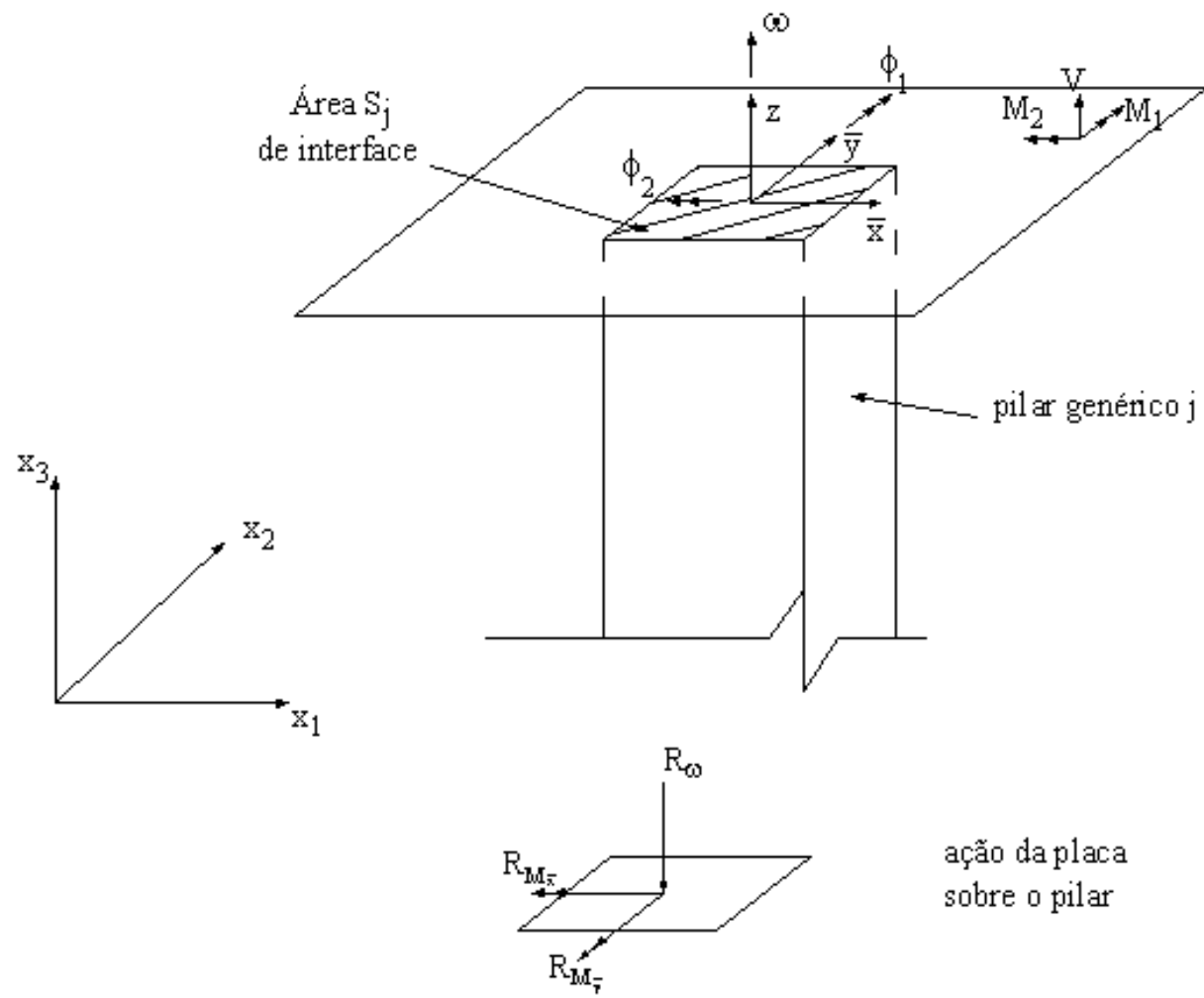

ação da placa sobre o pilar

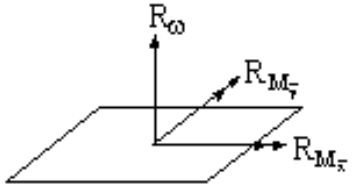

reaçăo do pilar sobre a placa

Figura 5.3 - Associação placa-pilar, eixos coincidentes com as direções principais de inércia

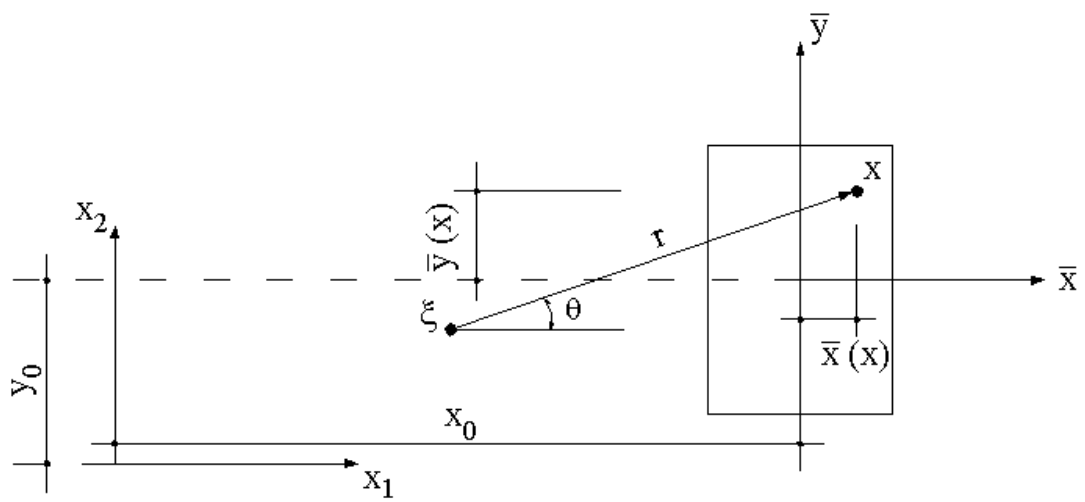

Figura 5.4 - Relações entre os sistemas de referência 


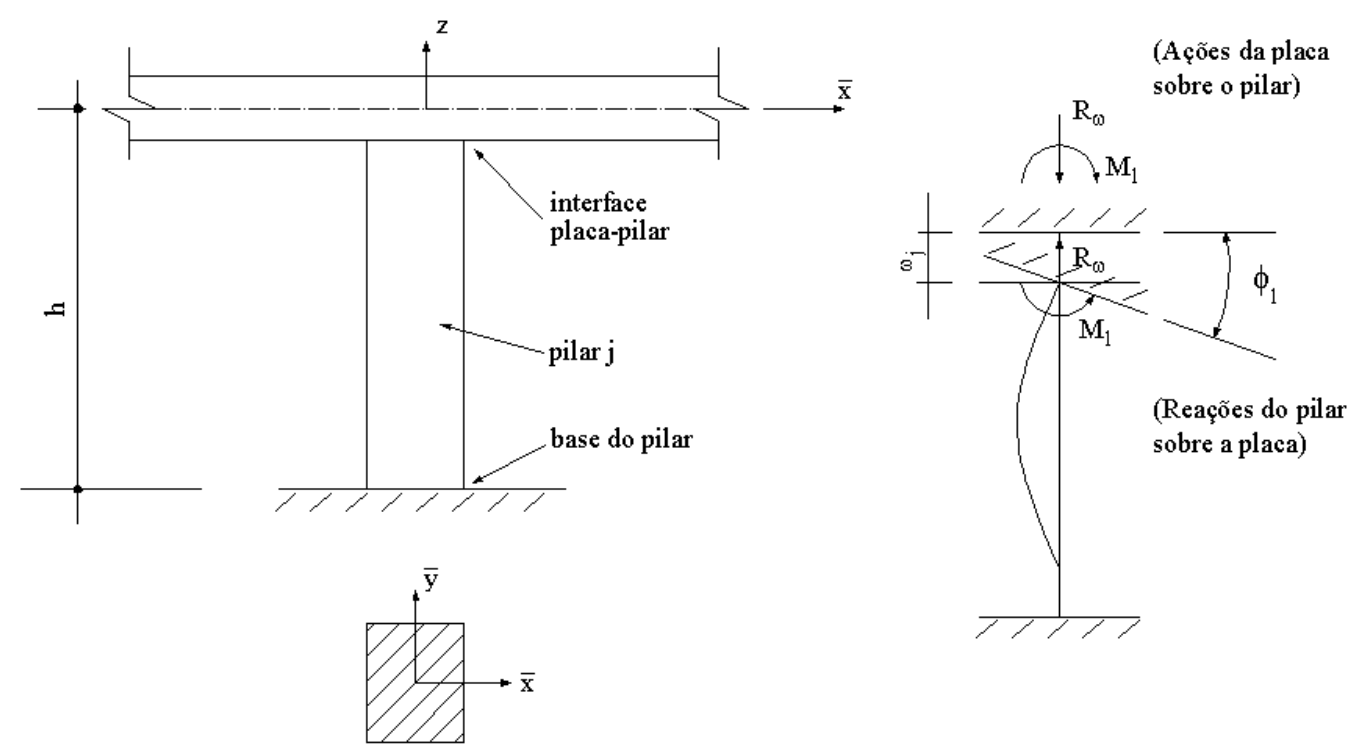

Figura 5.5 - Giros na interface placa-pilar

A tensão num ponto “x” do pilar, conforme figura (5.4) é dada por:

$$
\sigma(x)=\sigma_{0}+\frac{M_{\bar{y}}}{I_{\overline{y y}}} \bar{x}+\frac{M_{\bar{x}}}{I_{\overline{x x}}} \bar{y}
$$

onde:

$M_{\bar{x}}, M_{\bar{y}}$ - momentos fletores no pilar

$I_{\overline{x x}}, I_{\overline{y y}} \quad$ - momentos principais de inércia do pilar

$\sigma_{0}=\frac{R_{w}}{S}$ - tensão devida ao esforço normal no pilar

Considerando a figura (5.5) e analisando o pilar temos: 


$$
\begin{aligned}
& M_{\bar{x}}=-\frac{k_{p} E_{p} I_{\overline{x x}}}{l_{p}} \phi_{\bar{x}} \\
& M_{\bar{y}}=-\frac{k_{p} E_{p} I_{\overline{y y}}}{l_{p}} \phi_{\bar{y}} \\
& R_{w}=-\frac{E_{p} S_{p}}{l_{p}} w
\end{aligned}
$$

onde:

$E_{p}$ - módulo de elasticidade do pilar

$S_{p}$ - área da seção do pilar

$l_{p}$ - altura do pilar

$k_{p}$ - coeficiente que vale 3 para pilar articulado na base

e 4 para pilar engastado na base

O sinal negativo ocorre porque as reações tem sempre sinais opostos aos deslocamentos.

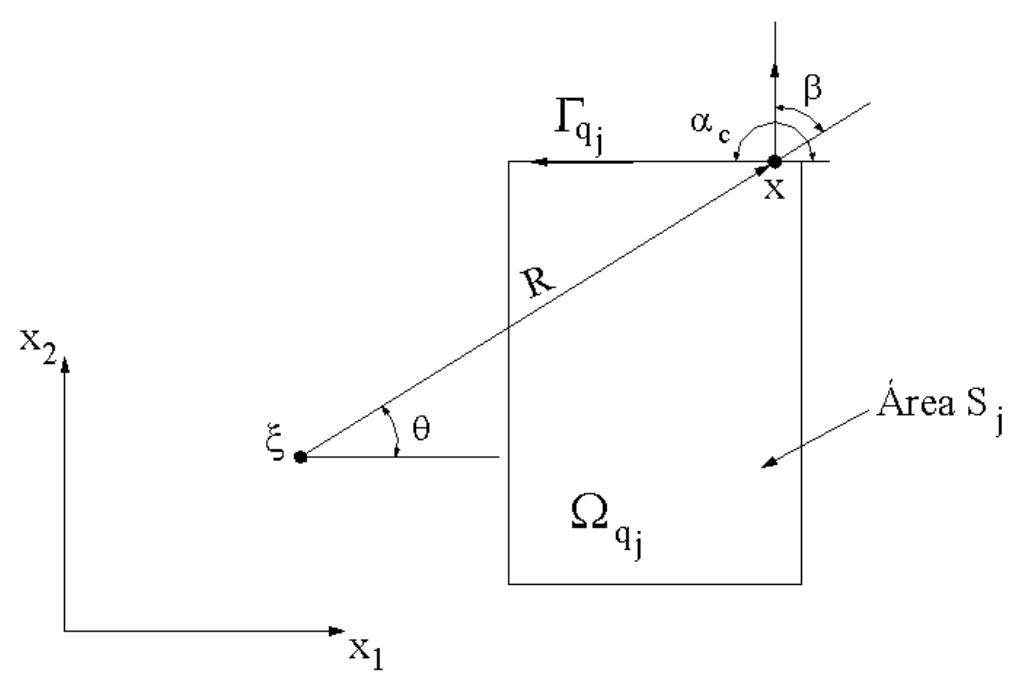

Figura 5.6 - Área do pilar com carga linearmente distribuída 
Calculando agora as tensões no ponto x em função das coordenadas de um ponto qualquer $\xi$ mostrado nas figuras (5.4) e (5.6) temos:

$$
\begin{gathered}
\sigma(x)=\frac{k_{p} E_{p}}{l_{p}}\left[x(\xi)-x_{0}+r \cos \theta\right] \phi_{1}+ \\
\frac{k_{p} E_{p}}{l_{p}}\left[y(\xi)-y_{0}+r \operatorname{sen} \theta\right] \phi_{2}-\frac{E_{p}}{l_{p}} w
\end{gathered}
$$

A influência da carga linearmente distribuída dada pela equação (5.8) atuando na área dos pilares é dada pela integral:

$$
I_{i}=\int_{\Omega_{S_{j}}} \sigma(x)\left[u_{i 3}^{*}(\xi, x)-\frac{v}{(1-v) \lambda^{2}} u_{i \alpha, \alpha}^{*}(\xi, x)\right] d \Omega_{S_{j}}
$$

Esta integral sobre o domínio, com convenientes operações, é transformada numa integral no contorno do pilar mostrado na figura (5.6) .

Considerando-se uma placa com NAP apoios internos conforme o apoio “j” mostrado podemos escrever em forma matricial:

$$
\mathbf{H U}=\mathbf{G P}+\mathbf{B}+\mathbf{Q U}_{\mathrm{s}}+\mathbf{E M}^{\mathbf{0}}
$$

onde a matriz Q(3NNCP, 3NAP) contém os coeficientes dados pela integral (5.9) após transformada para o contorno e o vetor $\mathbf{U}_{\mathbf{s}}(\mathbf{3 N A P})$ contém os 3 deslocamentos para cada um dos apoios internos. Considerando que os deslocamentos da interface são idênticos aos da placa na interface temos:

$$
\overline{\mathbf{U}}=\mathbf{U}_{\mathrm{s}}
$$

Para solução do sistema escrevemos (3NAP) novas equações de deslocamentos, agora escritas para a placa, nos pontos correspondentes ao centro de gravidade de cada um dos pilares.

$$
\overline{\mathbf{U}}=\overline{\mathbf{G}} \mathbf{P}-\overline{\mathbf{H}} \mathbf{U}+\overline{\mathbf{B}}+\overline{\mathbf{Q}} \overline{\mathbf{U}}+\overline{\mathbf{E}} \mathbf{M}^{0}
$$


Não há problemas de singularidade pois os CGs não pertencem ao contorno do pilar.

Montando uma única equação matricial com as (5.10) e (5.11) temos:

$$
\left[\begin{array}{ll}
\mathbf{H} & -\mathbf{Q} \\
\overline{\mathbf{H}} & \mathbf{I}-\overline{\mathbf{Q}}
\end{array}\right]\left\{\begin{array}{l}
\mathbf{U} \\
\overline{\mathbf{U}}
\end{array}\right\}=\left[\begin{array}{l}
\mathbf{G} \\
\overline{\mathbf{G}}
\end{array}\right] \mathbf{P}+\left\{\begin{array}{l}
\mathbf{B} \\
\overline{\mathbf{B}}
\end{array}\right\}+\left[\begin{array}{l}
\mathbf{E} \\
\overline{\mathbf{E}}
\end{array}\right] \mathbf{M}^{0}
$$

Resolvendo o sistema obtém-se os deslocamentos no contorno e nos pontos correspondentes aos apoios internos. Com os valores dos deslocamentos nos apoios e as equações (5.7) obtém-se as reações nos pilares. Com a equação (5.8) pode ser obtida a distribuição linear de tensões em cada apoio.

Deve-se dar preferência ao uso de apoios em áreas discretas, ao invés de apoios pontuais pois conduzem a melhores resultados, evitando algumas singularidades que poderiam ocorrer caso as cargas fossem concentradas.

\subsection{ASSOCIAÇÃO DA PLACA COM ESTRUTURA QUALQUER}

Neste item será analisada a associação de uma placa com uma estrutura qualquer formada por barras, trabalhando à flexão, constituindo uma grelha(ou no caso mais geral um pórtico 3D. No caso real a ligação entre a placa e a grelha é total e, para sua análise, deve-se considerar uma única estrutura tridimensional.

Nos trabalhos referentes às análises de edifícios, os pavimentos são considerados como estruturas bidimensionais (placas com efeitos de flexão e de membrana), para maior simplicidade, e ainda considerando-se que as ligações entre a placa e os elementos lineares (grelha) se dêem em linhas(linhas de carga) e pontos discretos (cargas concentradas). As linhas representam as vigas associadas as placas com rigidezes a torção e flexão, enrijecendo a placa.

Os esforços que aparecem na interface das duas estruturas podem ser interpretados como linhas de carga vertical e linha de momentos aplicados à placa. 


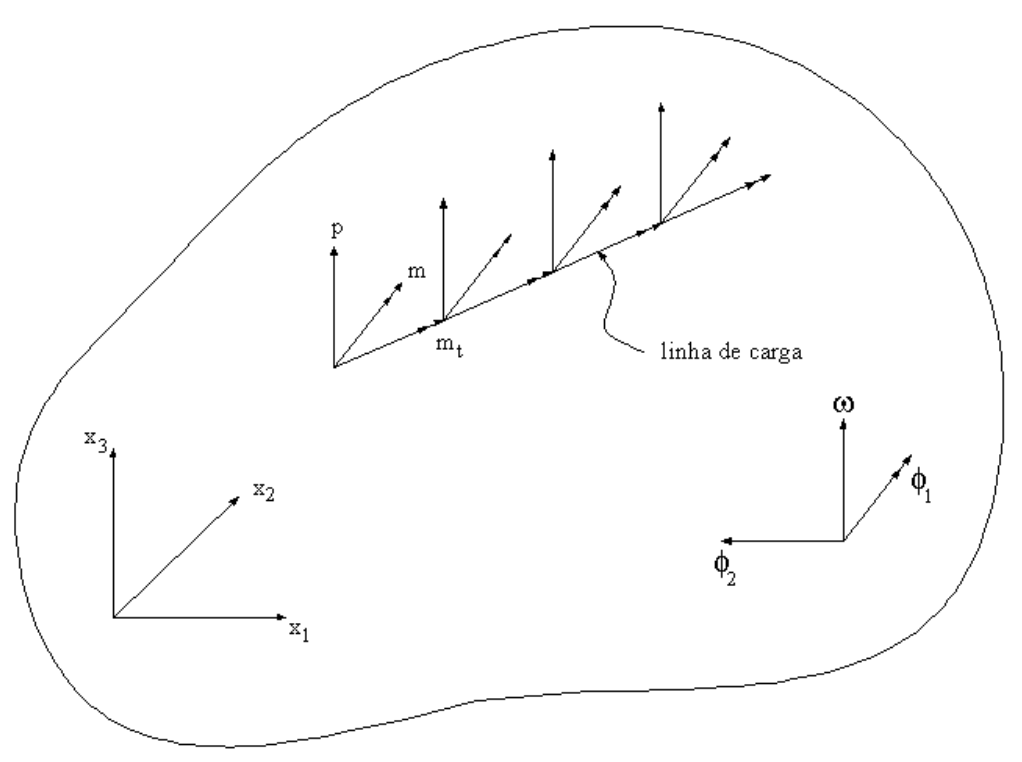

Figura 5.7 - Placa com linhas de cargas verticais e momentos

No nosso caso, as vigas serão representadas unicamente por linhas de carga vertical como as cargas $\mathbf{p}$ da figura 5.7. Este é um modelo bastante simplificado, porém que dá resultados precisos. Para esse modelo se obterá a solução exata da combinação elemento linear-placa quando o número de elementos utilizados na interface tende ao infinito. Uma justificativa que deve ser utilizada para a utilização de um modelo simples é relativa a problemas da formulação que podem ocorrer quando os graus de liberdade rotação também são considerados no acoplamento. Nesse caso, é necessário analisar as singularidades decorrentes da aplicação de momentos distribuídos em linha. A consideração das rotações e das forças de superfície conjugadas fazem com que algumas representações integrais a serem utilizadas seja singulares, requerendo maior cuidado na seleção dos nós que evitem singularidades reais (físicas) que começam a surgir. Por exemplo, as rotações para momentos aplicados em linha são singulares no final da linha de carga, portanto, necessariamente a linha de carga deve terminar em nó descontínuo. A integração desses termos vai resultar valores de Cauchy que devem ser cuidadosamente calculados analiticamente. E por último, os esforços internos ficam 
indeterminados, o que deve sempre ser evitado para dar uma generalização melhor ao algoritmo desenvolvido (robustez).

A representação integral dos deslocamentos (equações (3.2) e (3.3) para pontos internos e do contorno) onde são considerados campos de momentos iniciais e linhas de cargas no domínio da placa, podem ser transformadas em equações algébricas, que resulta no seguinte sistema de equações quando escritas para um número de pontos de colocação previamente definidos.

$$
\mathbf{H U}=\mathbf{G P}+\mathbf{B}+\mathbf{S p}^{\mathbf{N}}+\mathbf{E} \mathbf{M}^{\mathbf{0}}
$$

onde:

$\mathbf{p}^{\mathbf{N}}$ - vetor dos valores nodais das linhas de carga vertical, que representam as reações da estrutura sobre a placa (apenas a componente perpendicular ao plano da placa)

S - matriz cujos coeficientes representam as contribuições das forças distribuídas em linhas nos deslocamentos dos nós do contorno

A carga vertical distribuída atuante em cada elemento da linha de cargas (lembrar que os elementos de viga contém três nós necessários a aproximação dos deslocamentos e apenas uma carga vertical distribuída), será adotada constante. Assim, o vetor $\mathbf{p}$ referido a um elemento da linha de cargas será dado por um único valor $\mathbf{p}_{\mathbf{j}}$.

Desta forma, o vetor correspondente à linha de carga para um elemento genérico de carga “j” será dado por:

$$
\mathbf{p}_{\mathbf{j}}^{\mathbf{N}}=\{\mathbf{p}\}_{\mathbf{j}}
$$

Os deslocamentos nodais associados ao vetor $\mathbf{p}^{\mathbf{N}}$ são dados por:

$$
\mathbf{u}_{\mathbf{j}}^{\mathbf{N}}=\left\{\gamma^{1} \theta^{1} w^{1} \gamma^{2} \theta^{2} w^{2} \gamma^{3} \theta^{3} w^{3}\right\}
$$

O sistema local de referência para um elemento da linha de cargas está ilustrado na figura (5.8). A direção 1 corresponde ao eixo da barra e está associada aos momentos torçores da viga e à correspondente rotação $\gamma$, a direção 2 é perpendicular ao eixo da 
barra e está associada aos momentos fletores da viga e a correspondente rotação $\theta$, enquanto que a direção 3 coincide com a direção global $\mathbf{x}_{3}$ e está associada ao deslocamento transversal $\mathbf{w}$.

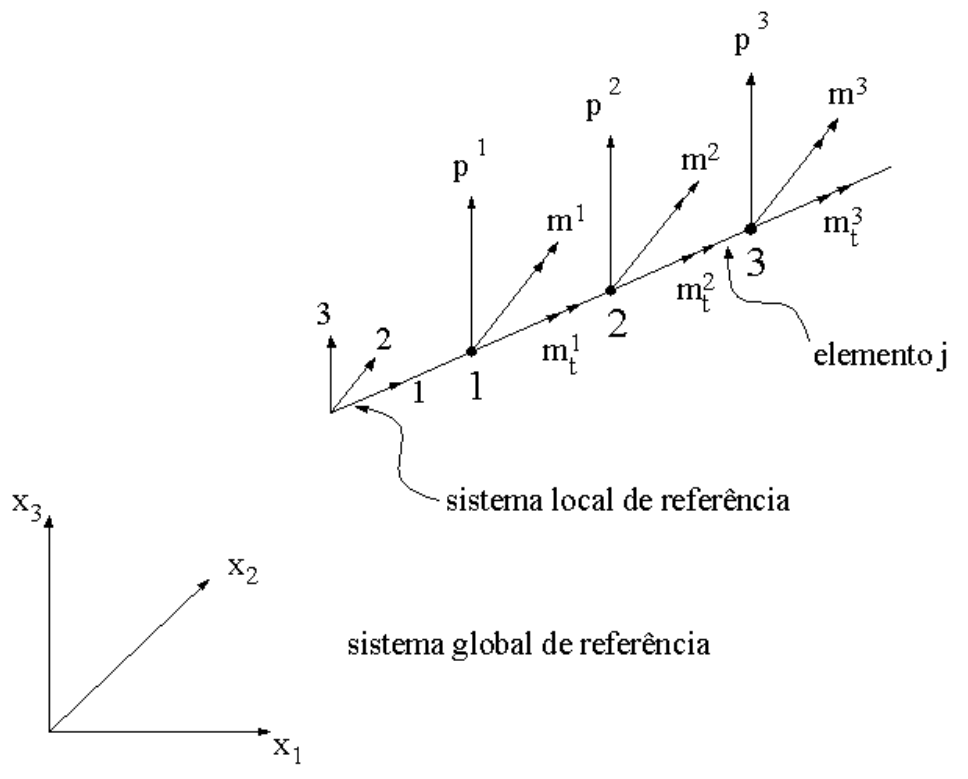

Figura 5.8 - Elemento “j” genérico na ligação placa estrutura

A matriz $\mathbf{S}$ da equação (5.13) representa a influência da linha de carga sobre os deslocamentos e possui 3NNCP linhas correspondentes a igual valor de deslocamentos do contorno e NBAR colunas, cada uma correspondente a um elemento de barra das vigas.

Considerando um ponto do contorno e um elemento “j” de viga (há NBAR destes elementos), a matriz $\mathbf{S}_{\mathbf{j}}$ é matriz coluna $(1,3)$ sendo seus coeficientes dados por:

$$
\begin{aligned}
& S_{\alpha}=\int_{\Gamma_{j}} u_{3 \alpha}^{*} d \Gamma_{j} \\
& S_{3}=\int_{\Gamma_{j}}\left[u_{33}^{*}-\frac{v}{(1-v) \lambda^{2}} u_{3 \alpha, \alpha}^{*}\right] d \Gamma_{j}
\end{aligned}
$$


Não haverá ocorrência de singularidade uma vez que os pontos de integração referentes ao contorno foram, em nosso caso, levados para fora da placa. As integrais (5.16) são todas resolvidas numericamente.

Analisando a equação (5.13) vemos que, além das incógnitas de contorno, aparecem também as cargas distribuídas representadas pelo vetor $\mathbf{p}$. Para o problema em análise, estas cargas representam as forças de interação entre os dois elementos estruturais; não são cargas usuais aplicadas ao sistema. Com a aproximação adotada, vão apresentar descontinuidade entre elementos. No caso de haver duas ou mais vigas que se cruzam num único ponto, a aproximação adotada vai garantir a independência das forças de interação.

Fazendo-se necessárias mais equações, escreve-se então a equação de deslocamentos dos pontos internos, para todos os NIPV pontos internos de viga.

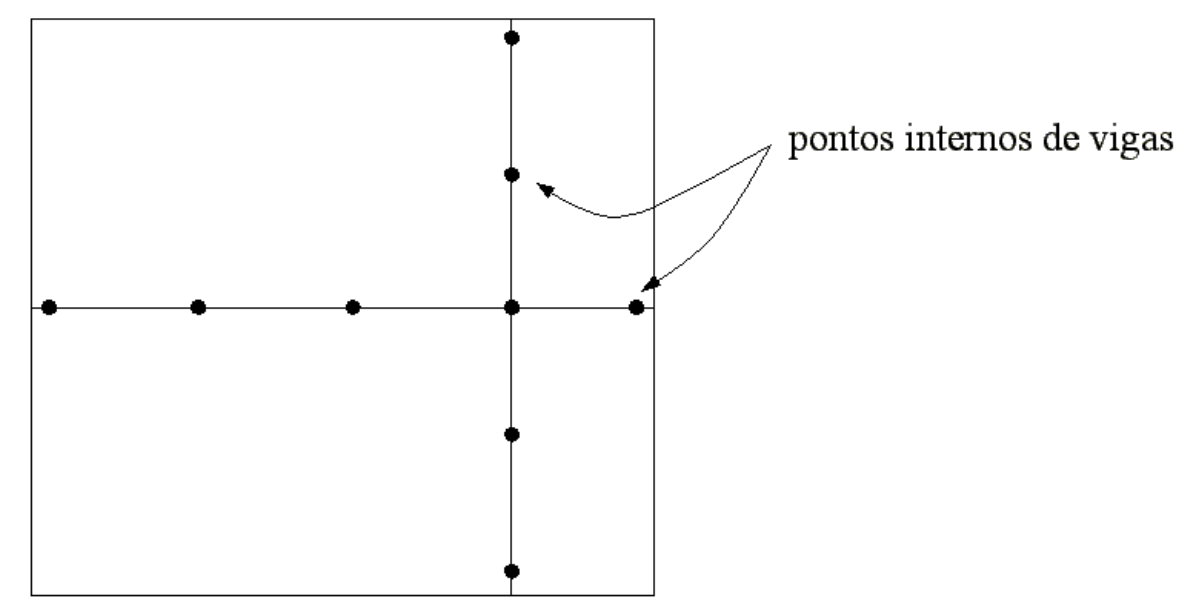

Figura 5.9 - Esquema dos NIPV pontos internos de viga

A equação algébrica obtida para os pontos do interior que pertencem à região de acoplamento, a partir da discretização da equação integral, pode ser representada em sua forma matricial por:

$$
\mathbf{w}=-\overline{\mathbf{H}} \mathbf{U}+\overline{\mathbf{G}} \mathbf{P}+\overline{\mathbf{S}} \mathbf{p}+\overline{\mathbf{E}} \mathbf{M}^{\mathbf{0}}
$$


A matriz $\overline{\mathbf{S}}$ possui NPIV linhas correspondentes cada uma a um dos NPIV pontos internos de viga e NBAR colunas. Note que apenas a equação da deflexão está representada em (5.17). Assim, como os NIPV pontos recebem apenas cargas verticais utiliza-se agora apenas a segunda das expressões (5.16) com seu valor $\mathbf{S}_{\mathbf{3}}$.

Caso o ponto de integração não coincida com nenhum dos pontos do elemento a ser integrado, as integrais podem ser calculadas numericamente, com ou sem o esquema de sub-elementação já descrito, utilizando-se pontos de Gauss. O cálculo das integrais analíticas para este caso é também relativamente simples.

Se o ponto de integração coincidir com um dos nós do elemento a ser integrado, recomenda-se que as integrais sejam calculadas analiticamente. Neste caso temos três situações diferentes.

Quando o ponto de integração coincide com o ponto nodal $\mathbf{k}_{\mathbf{2}}$, conforme representado abaixo, temos:

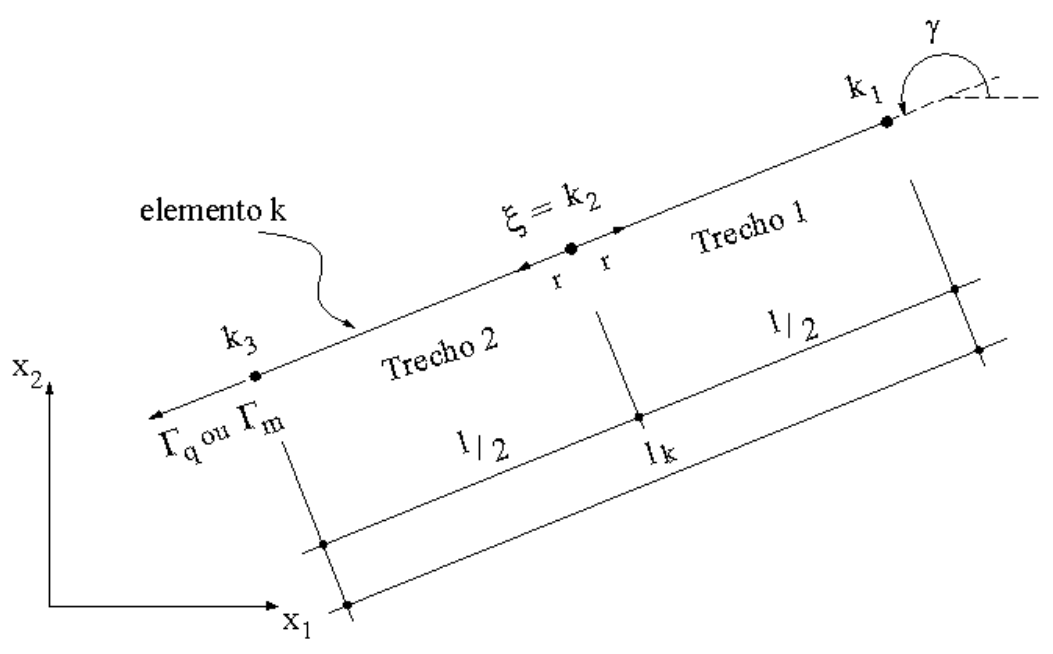

Figura 5.10 - Elemento de carga com $\xi=\mathbf{k}_{\mathbf{2}}$

Analisando-se a figura acima vem:

Trecho 1 


$$
\begin{aligned}
& r=-\Gamma=-\frac{l}{2} \eta \\
& d r=-d \Gamma=-\frac{l}{2} d \eta
\end{aligned}
$$

Trecho 2

$$
\begin{aligned}
& r=\Gamma=\frac{l}{2} \eta \\
& d r=d \Gamma=\frac{l}{2} d \eta
\end{aligned}
$$

A contribuição da linha de carga na determinação dos deslocamentos internos é então dada pela expressão de $\mathbf{S}_{\mathbf{3}}$, equação (5.16) :

$$
\begin{aligned}
& I_{q_{L}}=\int_{\Gamma q} q_{L}\left[u_{33}^{*}-\frac{v}{(1-v) \lambda^{2}} u_{3 \alpha, \alpha}^{*}\right] d \Gamma_{q}= \\
& \int_{0}^{l / 2} q_{L}\left[u_{33}^{*}-\frac{v}{(1-v) \lambda^{2}} u_{3 \alpha, \alpha}^{*}\right] d r_{1}+ \\
& \int_{0}^{l / 2} q_{L}\left[u_{33}^{*}-\frac{v}{(1-v) \lambda^{2}} u_{3 \alpha, \alpha}^{*}\right] d r_{2}
\end{aligned}
$$

Considerando que a carga $\mathbf{p}$ foi adotada constante para cada elemento, a integral acima, calculada analiticamente, é dada por:

$$
\begin{aligned}
& u_{33}^{*}=\frac{1}{8 \pi D(1-v) \lambda^{2}}\left[(1-v) z^{2}(\ln z-1)-8 \ln z\right] \\
& u_{3 \alpha, \alpha}^{*}=-\frac{\ln z}{2 \pi D}
\end{aligned}
$$




$$
\begin{aligned}
& I_{q_{L}}=\frac{1}{32 \pi D} L^{3}\left(\frac{1}{3} \ln \frac{\lambda L}{2}-\frac{4}{9}\right)+ \\
& \frac{(v-2)}{2 \pi D(1-v) \lambda^{2}} L\left(\ln \frac{\lambda L}{2}-1\right)
\end{aligned}
$$

Quando o ponto de integração coincide com o ponto nodal $\mathbf{k}_{\mathbf{1}}$, conforme representado abaixo, temos:

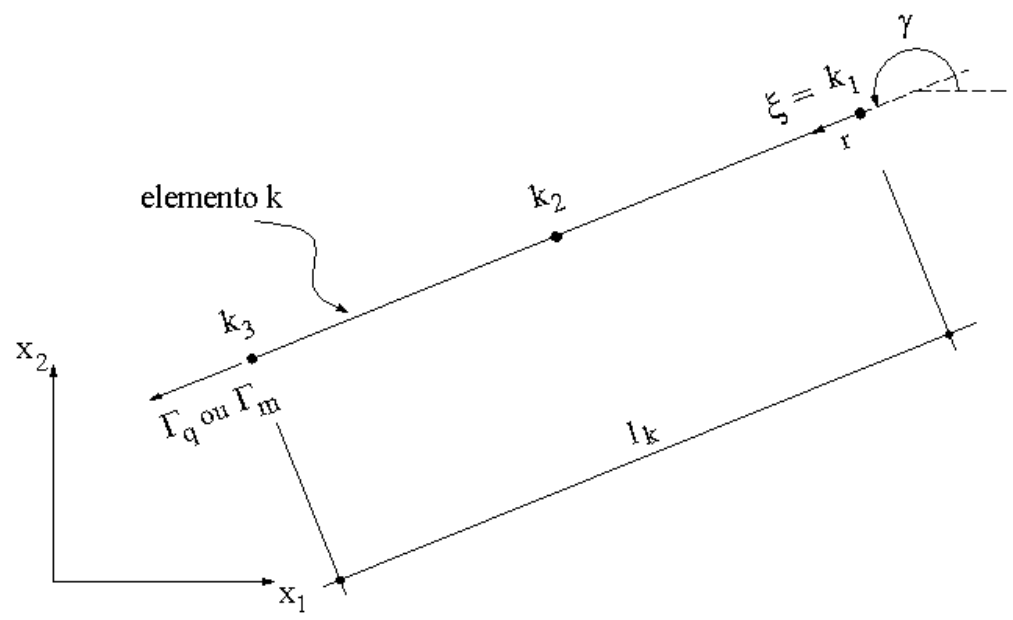

Figura 5.11- Elemento de carga com $\xi=\mathbf{k}_{\mathbf{1}}$

Analisando-se a figura temos:

$$
\begin{aligned}
& r=\Gamma+\frac{l}{2}=\frac{l}{2}(\eta+1) \\
& d r=d \Gamma=\frac{l}{2} d \eta
\end{aligned}
$$

A contribuição da linha de carga na determinação dos deslocamentos internos é então dada pela expressão de $\mathbf{S}_{\mathbf{3}}$ dada na (5.16): 


$$
\begin{aligned}
& I_{q_{L}}=\int_{\Gamma q} q_{L}\left[u_{33}^{*}-\frac{v}{(1-v) \lambda^{2}} u_{3 \alpha, \alpha}^{*}\right] d \Gamma_{q}= \\
& \int_{0}^{l} q_{L}\left[u_{33}^{*}-\frac{v}{(1-v) \lambda^{2}} u_{3 \alpha, \alpha}^{*}\right] d r
\end{aligned}
$$

Analogamente ao caso anterior, considerando a carga $\mathbf{p}$ constante para cada elemento, a integral acima, calculada analiticamente,é dada por:

$$
\begin{aligned}
& u_{33}^{*}=\frac{1}{8 \pi D(1-v) \lambda^{2}}\left[(1-v) z^{2}(\ln z-1)-8 \ln z\right] \\
& u_{3 \alpha, \alpha}^{*}=-\frac{\ln z}{2 \pi D}
\end{aligned}
$$

Efetuando-se a integração temos:

$$
\begin{aligned}
& I_{q_{L}}=\frac{1}{8 \pi D} L^{3}\left(\frac{1}{3} \ln \lambda L-\frac{4}{9}\right)+ \\
& \frac{(v-2)}{2 \pi D(1-v) \lambda^{2}} L(\ln \lambda L-1)
\end{aligned}
$$

Idêntica expressão pode ser usada para o cálculo quando $\xi=\mathbf{k}_{\mathbf{3}}$

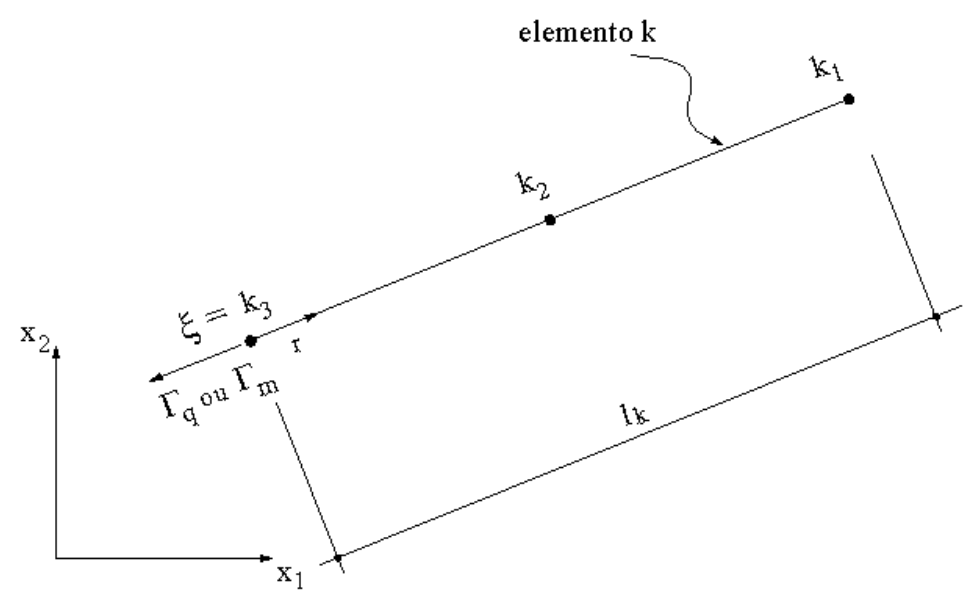

Figura 5.12 - Elemento de carga com $\xi=\mathbf{k}_{\mathbf{3}}$ 


\subsection{ELEMENTOS FINITOS DE VIGA}

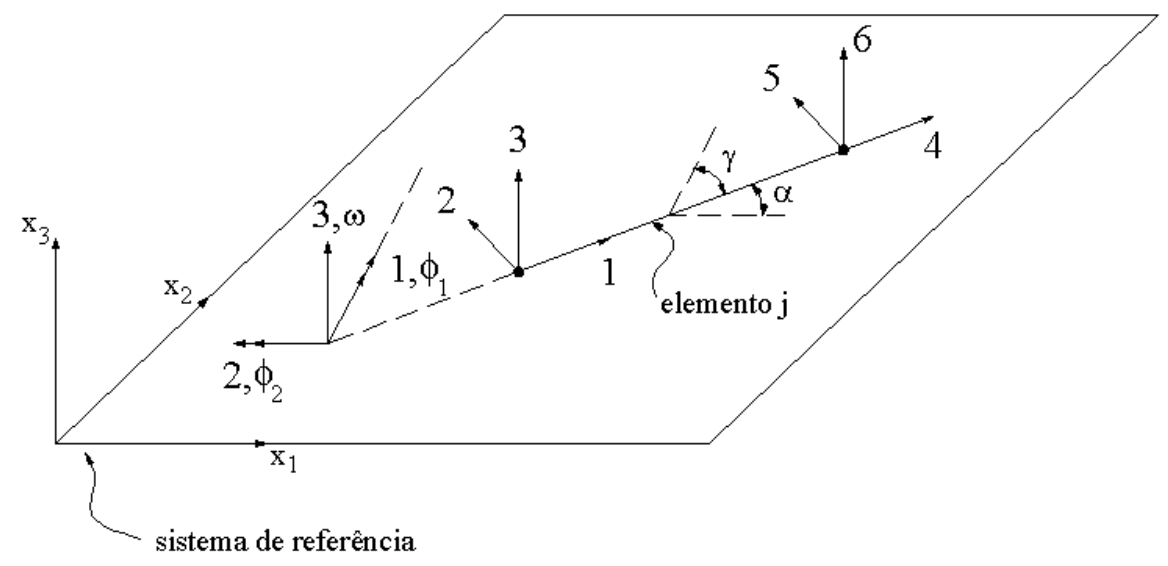

Figura 5.13 - Elemento finito e seus sistemas de referência

Considerando um elemento finito temos, conforme SAVASSI(1996), a seguinte relação escrita referida a um sistema local de coordenadas:

$$
f=k u
$$

onde:

$k$ - matriz de rigidez do elemento

$U$ - vetor de deslocamentos nodais do elemento

$f$ - vetor das forças nodais equivalentes do elemento

Para escrever a equação (5.20) em coordenadas globais considerando que as barras podem não estar alinhadas com o sistema de eixos global temos a seguinte transformação:

$$
\begin{aligned}
& f=A F \\
& u=A U
\end{aligned}
$$

onde: 
A - matriz de transformação de coordenadas locais para globais dada por

$$
A=\left[\begin{array}{cccccc}
C_{x} & C_{y} & 0 & 0 & 0 & 0 \\
-C_{y} & C_{x} & 0 & 0 & 0 & 0 \\
0 & 0 & 1 & 0 & 0 & 0 \\
0 & 0 & 0 & C_{x} & C_{y} & 0 \\
0 & 0 & 0 & -C_{y} & C_{x} & 0 \\
0 & 0 & 0 & 0 & 0 & 1
\end{array}\right]
$$

sendo:

$$
\begin{aligned}
& C_{x}=\cos \gamma \\
& C_{y}=\operatorname{sen} \gamma
\end{aligned}
$$

com o ângulo $\gamma$ dado na figura (5.13) correspondendo ao ângulo formado entre as direções 1 dos dois sistemas de coordenadas e $\alpha$ o ângulo que a direção da barra faz com o eixo global $\mathbf{x}_{\mathbf{1}}$.

Colocando os valores de (5.21) em (5.20) temos:

$$
A F=k A U
$$

Multiplicando a esquerda pela inversa de A, que é numericamente igual à sua matriz transposta temos:

$$
F=A^{T} k A U
$$

Fazendo $K=A^{T} k A$

Sabendo que: 
$k=\left[\begin{array}{cccccc}\frac{G J_{t}}{l} & 0 & 0 & -\frac{G J_{t}}{l} & 0 & 0 \\ 0 & \frac{4 E I}{l} & -\frac{6 E I}{l^{2}} & 0 & \frac{2 E I}{l} & \frac{6 E I}{l^{2}} \\ 0 & -\frac{6 E I}{l^{2}} & \frac{12 E I}{l^{3}} & 0 & -\frac{6 E I}{l^{2}} & -\frac{12 E I}{l^{3}} \\ -\frac{G J_{t}}{l} & 0 & 0 & \frac{G J_{t}}{l} & 0 & 0 \\ 0 & \frac{2 E I}{l} & -\frac{6 E I}{l^{2}} & 0 & \frac{4 E I}{l} & \frac{6 E I}{l^{2}} \\ 0 & \frac{6 E I}{l^{2}} & -\frac{12 E I}{l^{3}} & 0 & \frac{6 E I}{l^{2}} & \frac{12 E I}{l^{3}}\end{array}\right]$

Assim, a matriz $\mathbf{K}$ de uma barra de 2 nós referida às direções globais fica: $K=\left[\begin{array}{ll}{\left[K_{11}\right]} & {\left[K_{12}\right]} \\ {\left[K_{21}\right]} & {\left[K_{22}\right]}\end{array}\right]$

Onde as sub-matrizes de $\mathbf{K}$ são dadas por:

$$
K_{11}=\left[\begin{array}{ccc}
\frac{G J_{t} C_{x}^{2}+4 E I C_{y}^{2}}{l} & \left(\frac{G J_{t}-4 E I}{l}\right) C_{x} C_{y} & \frac{6 E I C_{y}}{l^{2}} \\
& \frac{G J_{t} C_{y}^{2}+4 E I C_{x}^{2}}{l} & -\frac{6 E I C_{x}}{l^{2}} \\
\operatorname{sim} & \frac{12 E I}{l^{3}}
\end{array}\right]
$$




$$
\begin{aligned}
& K_{22}=\left[\begin{array}{ccc}
\frac{G J_{t} C_{x}^{2}+4 E I C_{y}^{2}}{l} & \left(\frac{G J_{t}-4 E I}{l}\right) C_{x} C_{y} & -\frac{6 E I C_{y}}{l^{2}} \\
\frac{G J_{t} C_{y}^{2}+4 E I C_{x}^{2}}{l} & \frac{6 E I C_{x}}{l^{2}} \\
\operatorname{sim} & \frac{12 E I}{l^{3}}
\end{array}\right] \\
& K_{12}=\left[\begin{array}{ccc}
\frac{-G J_{t} C_{x}^{2}+2 E I C_{y}^{2}}{l} & -\left(\frac{G J_{t}+2 E I}{l}\right) C_{x} C_{y} & -\frac{6 E I C_{y}}{l^{2}} \\
-\left(\frac{G J_{t}+2 E I}{l}\right) C_{x} C_{y} & \frac{-G J_{t} C_{y}^{2}+2 E I C_{x}^{2}}{l} & \frac{6 E I C_{x}}{l^{2}} \\
\frac{6 E I C_{y}}{l^{2}} & -\frac{6 E I C_{x}}{l^{2}} & \frac{12 E I}{l^{3}}
\end{array}\right]
\end{aligned}
$$

A matriz K é simétrica, conforme já considerado acima.

Para termos incógnitas de mesmo tipo (ou forças concentradas como as nodais $\mathbf{F}$ da equação dos elementos finitos ou forças distribuídas na interface), precisamos transformar o vetor $\mathbf{F}$ das forças nodais equivalentes no vetor $\mathbf{p}$ dos valores nodais das forças distribuídas nos elementos de interface.

Isto é feito pela seguinte relação:

$$
\mathbf{F}=\mathbf{N p}
$$

Mas, a expressão original do MEF é escrita em função de coordenadas locais, então:

$$
\mathbf{f}=\mathbf{A}^{\mathrm{T}} \mathbf{F}=\mathbf{A}^{\mathrm{T}} \mathbf{N p}
$$

onde $\mathbf{N}$ pode ser calculada por: 


$$
N=\frac{l}{2} \int_{-1}^{1} \phi_{v}^{T}(\eta) \phi_{p}(\eta) d \eta
$$

que é função das aproximações adotadas para os deslocamentos e cargas:

$$
\begin{aligned}
& u(\eta)=\phi_{v}(\eta) u^{N} \\
& p(\eta)=\phi_{p}(\eta) p^{N}
\end{aligned}
$$

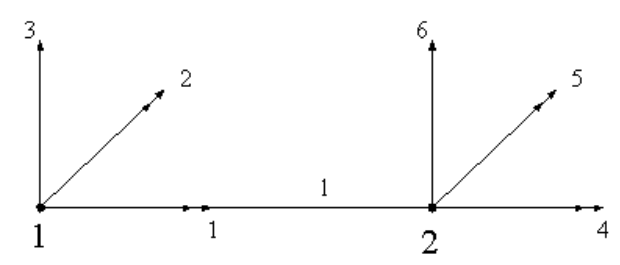

a)

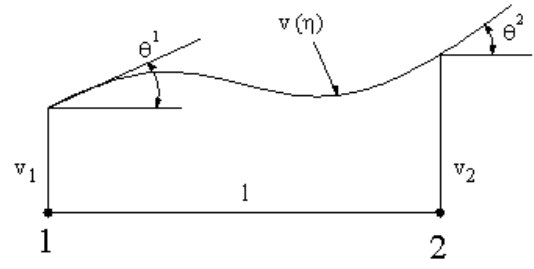

b)

Figura 5.14 - Definição das variáveis nodais para o elemento de barra com 2 nós

No nosso caso, adotando-se aproximação cúbica para os valores de $\mathbf{v}$ (ver figura acima) e carga $\mathbf{p}$ constante para cada elemento temos:

$$
u(\eta)=\left\{\begin{array}{l}
\gamma(\eta) \\
\theta(\eta) \\
u(\eta)
\end{array}\right\}=\phi_{v}(\eta) u^{N}=\left[\left[\phi_{1}(\eta)\right] \quad\left[\phi_{2}(\eta)\right]\right] u^{N}
$$

onde:

$$
\phi_{1}(\eta)=\left[\begin{array}{ccc}
\frac{1}{2}(1-\eta) & 0 & 0 \\
0 & \frac{1}{4}\left(-1-2 \eta+3 \eta^{2}\right) & \frac{-1}{2 l}\left(-3+3 \eta^{2}\right) \\
0 & \frac{-l}{8}\left(1-\eta-\eta^{2}+\eta^{3}\right) & \frac{1}{4}\left(2-3 \eta+\eta^{3}\right)
\end{array}\right]
$$




$$
\phi_{2}(\eta)=\left[\begin{array}{ccc}
\frac{1}{2}(1+\eta) & 0 & 0 \\
0 & \frac{1}{4}\left(-1+2 \eta+3 \eta^{2}\right) & \frac{-1}{2 l}\left(3-3 \eta^{2}\right) \\
0 & \frac{-l}{8}\left(-1-\eta+\eta^{2}+\eta^{3}\right) & \frac{1}{4}\left(2+3 \eta-\eta^{3}\right)
\end{array}\right]
$$

Considerando as expressões (5.26), (5.27), (5.28) e (5.29) temos que, para um elemento com 2 nós e carregamento p constante:

$$
\left(A^{T} N\right)^{T}=\left[\begin{array}{llllll}
\frac{C_{x} l^{2}}{12} & -\frac{C_{y} l^{2}}{12} & \frac{l}{2} & -\frac{C_{x} l^{2}}{12} & \frac{C_{y} l^{2}}{12} & \frac{l}{2}
\end{array}\right]
$$

O elemento de viga usado no trabalho possui 3 nós ao invés de 2. Nesta situação podemos imaginar este elemento como composto pela superposição de 2 elementos de 2 nós conforme mostrado abaixo.

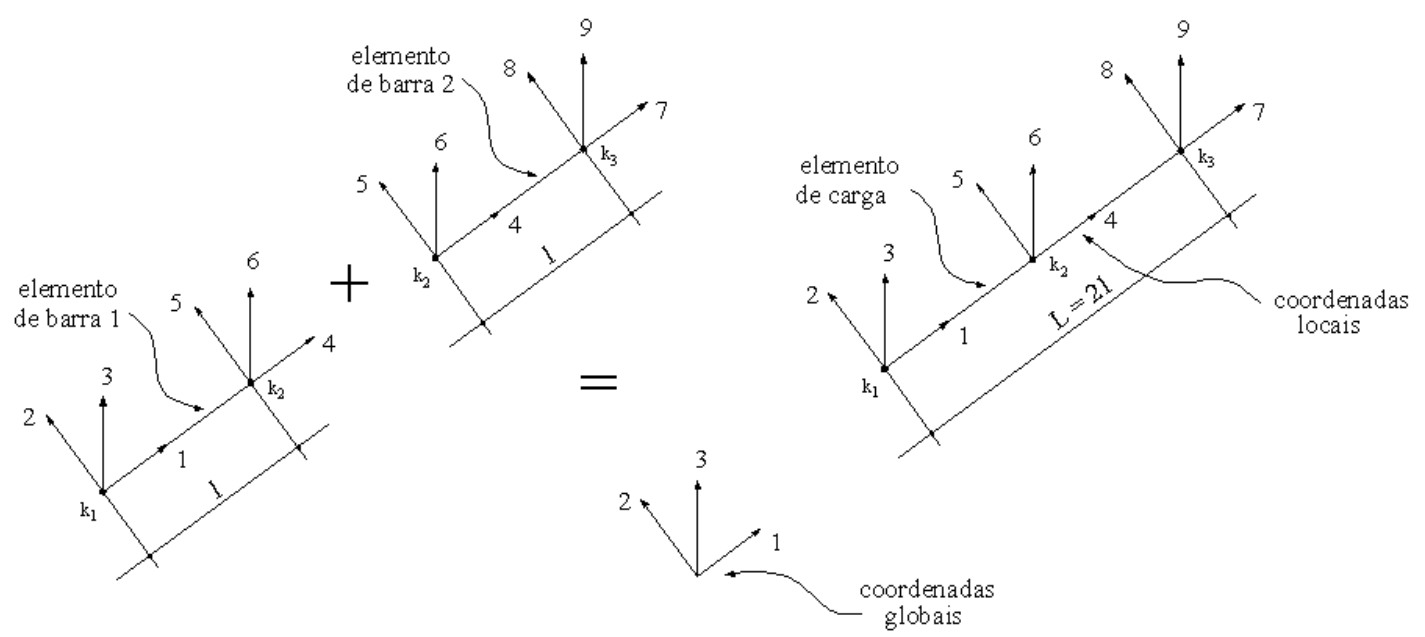

Figura 5.15 - Elementos de carga e de barra

A matriz K será dada por: 


$$
K_{\text {3nós }}=\left[\begin{array}{ccc}
{\left[K_{11}^{1}\right]} & {\left[K_{12}^{1}\right]} & 0 \\
{\left[K_{21}^{1}\right]} & {\left[K_{22}^{1}+K_{11}^{2}\right]} & {\left[K_{12}^{2}\right]} \\
0 & {\left[K_{21}^{2}\right]} & {\left[K_{22}^{2}\right]}
\end{array}\right]
$$

onde o cuidado principal a ser tomado refere-se ao comprimento $\mathrm{L}$ do novo elemento que é o dobro do anterior.

Analogamente para a matriz $\mathbf{N}$ temos:

$$
A^{T} N=\left\{\begin{array}{c}
\frac{C_{x} l^{2}}{12} \\
-\frac{C_{y} l^{2}}{12} \\
\frac{l}{2} \\
0 \\
0 \\
\frac{l}{C_{x} l^{2}} \\
\frac{12}{C_{y} l^{2}} \\
\frac{12}{l} \\
\frac{1}{2}
\end{array}\right\}
$$

\subsection{FORMULAÇÃO DO ACOPLAMENTO MEC/MEF}

Trataremos agora da formulação proposta do acoplamento do método dos elementos de contorno e do método dos elementos finitos para considerar as barras enrijecendo o domínio.

Para considerar o domínio bidimensional da placa modelada pelo MEC enrijecido com barras modeladas pelo MEF, é proposta uma formulação combinando-se 
as equações dos dois métodos. O acoplamento entre as peças é garantido pela imposição do equilíbrio de forças da interface e compatibilidade de deslocamentos ou seja:

$$
\begin{aligned}
& \mathbf{p}^{\mathbf{c}}+\mathbf{p}^{\mathbf{f}}=\mathbf{0} \\
& \mathbf{u}^{\mathbf{c}}=\mathbf{u}^{\mathbf{f}}
\end{aligned}
$$

onde os índices c e f referem-se ao MEC(placa) e MEF(barras).

A força da placa nas barras é igual e de sinal contrário a das barras na placa, enquanto que os deslocamentos dos dois elementos são iguais em módulo e sentido.

Assim, para o problema proposto temos as equações do MEC considerando as linhas de carga:

$$
\mathbf{H U}=\mathbf{G P}+\mathbf{B}+\mathbf{S p}^{\mathbf{N}}+\mathbf{E M}^{\mathbf{0}}
$$

que representam 3NNCP equações escritas para os deslocamentos dos nós do contorno e

$$
\mathbf{w}=-\overline{\mathbf{H}} \mathbf{U}+\overline{\mathbf{G}} \mathbf{P}+\overline{\mathbf{S}} \mathbf{p}+\overline{\mathbf{E}} \mathbf{M}^{\mathbf{0}}
$$

escrita para os NIPV nós associados às barras internas. Caso os nós das barras coincidam com os nós do contorno, as equações não necessitam ser reescritas, pois os deslocamentos destes já tem suas equações inseridas na equação (5.33). Quando os elementos de barra começam no contorno, será adotado para início desse elemento um novo número de nó com as mesmas coordenadas do nó do contorno, fazendo-se a compatibilidade dos deslocamentos destes dois nós. 


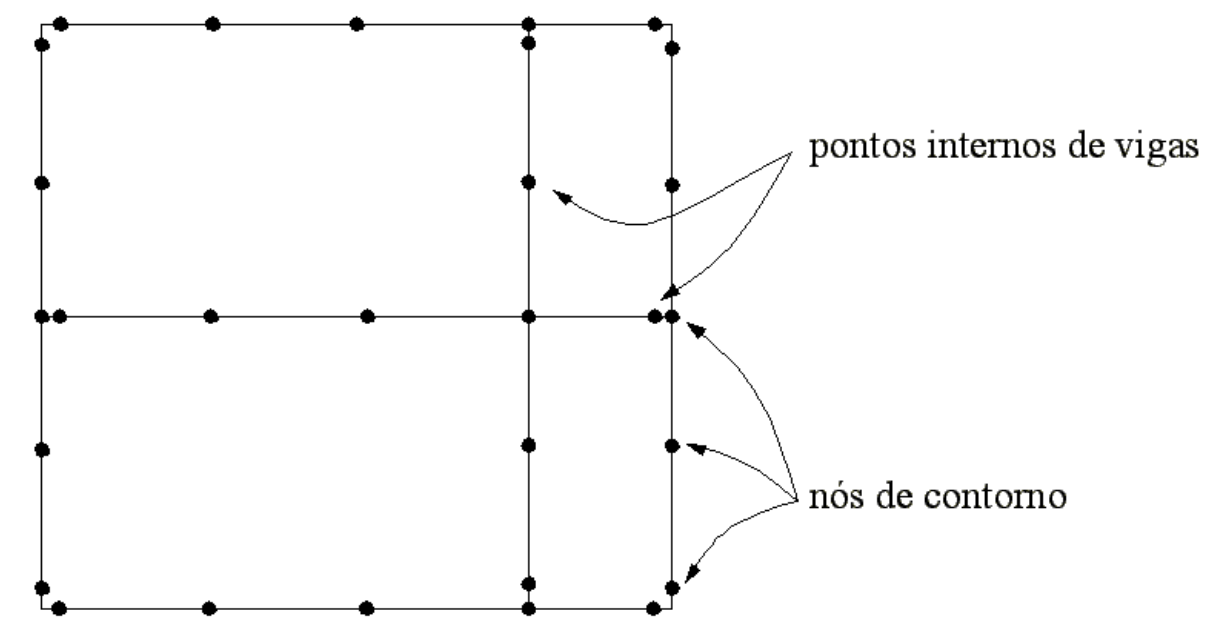

Figura 5.16 - Esquema dos nós das barras internas

Para a estrutura composta por barras, i.e. a grelha, a partir das equações do método dos elementos finitos escreve-se o seguinte sistema de equações algébricas:

$$
\mathbf{K}^{\mathrm{f}} \mathbf{U}^{\mathrm{f}}=\mathbf{A}^{\mathrm{T}} \mathbf{N} \mathbf{p}^{\mathrm{f}}
$$

Neste sistema há três equações algébricas por nó. O vetor de valores nodais contém também três variáveis por nó, a deflexão w, já introduzida na equação algébrica do MEC, equação (5.32), e mais duas rotações.

\subsection{COMBINAÇÃO DAS EQUAÇÕES DO MEC/MEF COM REGULARIZAÇÃO PELO MÉTODO DOS MÍNIMOS QUADRADOS}

Considerando o sistema de equações formado pelas as equações (5.33), (5.34) e (5.35), sem esquecer as compatibilidades dadas por (5.32), temos um sistema algébrico com mais equações do que incógnitas. A causa deste fato é simples e vem da adoção de diferentes graus para os polinômios aproximadores das forças da interface e dos deslocamentos. 
Para que este sistema de equações tenha solução, utiliza-se um procedimento simples baseado no método dos mínimos quadrados. Como o número de equações é maior que o número de incógnitas(equações redundantes) é preciso reduzi-lo a um número conveniente. A técnica dos mínimos quadrados consiste em reduzir o número de equações tornando-o igual ao número de incógnitas, minimizando ao mesmo tempo o erro da resposta quando levada ao sistema original. Note-se que as equações de equilíbrio não são exatamente atendidas; são aproximadamente atendidas com erro mínimo. Observando a equação (5.34) podemos fazer isto multiplicando-a pela matriz $\overline{\mathbf{S}}^{T}$,transposta da matriz $\overline{\mathbf{S}}$ ou seja:

$$
\overline{\mathbf{S}}^{\mathbf{T}} \mathbf{W}=-\overline{\overline{\mathbf{S}}}^{\mathbf{T}} \mathbf{H} \mathbf{U}+\overline{\mathbf{S}}^{\mathbf{T}} \overline{\mathbf{G}} \mathbf{P}+\overline{\mathbf{S}}^{\mathbf{T}} \overline{\mathbf{S}} \mathbf{p}+\overline{\mathbf{S}}^{\mathbf{T}} \overline{\mathbf{E}} \mathbf{M}^{\mathbf{0}}
$$

O novo sistema formado pelas (5.33), (5.35) e (5.36) tem um número de incógnitas igual ao número de equações e igual a:

$3 \mathrm{NNCP}+\mathrm{NBAR}+3 \mathrm{NIPV}$ onde

3NNCP - correspondentes aos deslocamentos dos nós do contorno

NBAR - correspondentes ao número das barras da grelha, cada qual possui uma única carga distribuída

3NIPV - correspondentes aos 3 deslocamentos por nó, dos nós dos pontos internos que pertencem às vigas

Reunindo as equações num único sistema e separando as incógnitas fica:

$$
\begin{aligned}
& {\left[\begin{array}{cccc}
\mathbf{H} & \mathbf{0} & -\mathbf{S} & \mathbf{0} \\
\overline{\mathbf{S}}^{\mathbf{T}} \overline{\mathbf{H}} & \overline{\mathbf{S}}^{\mathbf{T}} & -\overline{\mathbf{S}}^{\mathbf{T}} \overline{\mathbf{S}} & \mathbf{0} \\
\mathbf{K}_{\mathbf{u}} & \mathbf{K}_{\mathbf{w}} & \mathbf{A}^{\mathbf{T}} \mathbf{N} & \mathbf{K}_{\varphi}
\end{array}\right]\left\{\begin{array}{l}
\mathbf{U} \\
\mathbf{w} \\
\mathbf{p} \\
\boldsymbol{\varphi}
\end{array}\right\}=\left[\begin{array}{c}
\mathbf{G} \\
\overline{\mathbf{S}}^{\mathbf{T}} \overline{\mathbf{G}} \\
\mathbf{0}
\end{array}\right]\{\mathbf{P}\}} \\
& +\left\{\begin{array}{l}
\mathbf{B} \\
\overline{\mathbf{S}}^{\mathbf{T}} \overline{\mathbf{B}} \\
\mathbf{0}
\end{array}\right\}+\left[\begin{array}{c}
\mathbf{E} \\
\overline{\mathbf{S}}^{\mathbf{T}} \overline{\mathbf{E}} \\
\mathbf{0}
\end{array}\right] \mathbf{M}^{\mathbf{0}}
\end{aligned}
$$


onde a matriz $\mathbf{K}$ proveniente do método dos elementos finitos foi aqui subdividida em 3 para considerar o fato de que os deslocamentos a que ela se refere poderem pertencer aos deslocamentos $\mathbf{U}$ do contorno, ou aos deslocamentos $\mathbf{w}$ dos pontos internos da grelha ou aos deslocamentos angulares $\phi$ também referentes aos pontos internos da grelha.

Com as variáveis obtidas na solução do sistema (5.37) calculam-se deslocamentos em outros pontos e esforços em qualquer ponto interno, a partir das equações (4.18), onde nos termos B' e B'" dessas dão agora os efeitos das forças de interface p distribuídas e referentes ao enrijecimento causado pelo efeito da grelha.

Deve ser notado que, embora tenha sido utilizada no acoplamento apenas a força distribuída, a força cortante não é unicamente definida nos pontos da interface. Para a sua determinação seria necessário distribuir a força da interface em uma área de espessura dependente da largura da viga. 


\section{MODELOS CONSTITUTIVOS}

\subsection{INTRODUÇÃO}

São aqui considerados, de forma resumida os conceitos da teoria da plasticidade e da mecânica de dano, utilizados a seguir na modelagem de pavimentos de concreto armado.

\subsection{TEORIA DA PLASTICIDADE}

A Teoria da Plasticidade tem como objetivo estabelecer relações entre tensões e deformações que possibilitem, dado um determinado estado de tensões num ponto, calcular as deformações elásticas e plásticas do material neste ponto.

A Teoria da Plasticidade é ideal para materiais dúcteis, como o aço, mas é também usada para materiais frágeis, como o concreto, de maneira aproximada. Para materiais frágeis, a ruptura, que ocorre devido a abertura de fissuras, acontece sem plastificação significativa, pois estes materiais tem pequena capacidade de deformação, ao contrário dos materiais dúcteis.

A modelagem do comportamento elasto-plástico de um material necessita de relações entre tensão e deformação nas fases elástica e plástica, esta ocorrendo após o escoamento.

É necessário também uma lei, que explicite a evolução do tensor de deformações plásticas, bem como um critério de plastificação que indique quando o escoamento 
ocorrerá e, no caso de modelos que considerem o encruamento deve-se ter uma regra de endurecimento que dará valores da tensão de escoamento em função das deformações plásticas.

Considerando o caso uniaxial, representado na prática pelo ensaio de tração simples, podemos distinguir 2 modelos principais: o modelo elastoplástico perfeito e o modelo elastoplástico com encruamento. Outras possibilidades surgem no caso de carregamento multiaxial.

\subsubsection{MODELO ELASTOPLÁSTICO PERFEITO}

Neste modelo, ignora-se o efeito de endurecimento após atingida a tensão de escoamento. Admite-se que deformações plásticas ocorrem quando a tensão alcança o valor da tensão de escoamento, para valores menores da tensão atuante as deformações são totalmente elásticas. Nunca ocorrem tensões superiores à tensão de escoamento.

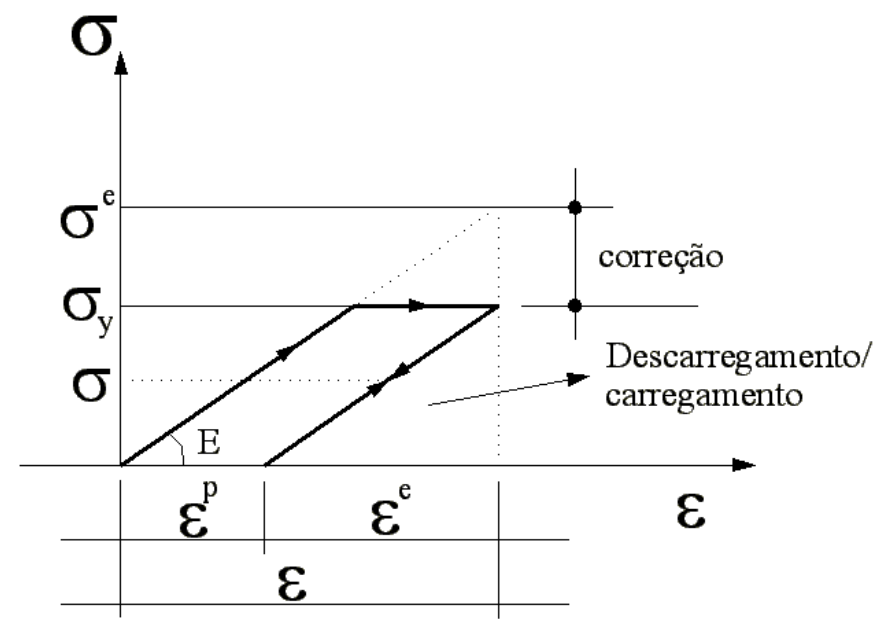

Figura 6.1 - Modelo elastoplástico perfeito

Neste caso o critério de plastificação é dado por:

$$
f(\sigma)=|\sigma|-\sigma_{y} \leq 0
$$

onde

$\sigma \quad$ é a tensão a que o ponto está submetido e $\sigma_{y}$ é a tensão de escoamento do material. 
A deformação total, por sua vez, pode ser dividida em uma parcela de deformação elástica e uma parcela de deformação plástica, ou seja:

$$
\varepsilon=\varepsilon^{e}+\varepsilon^{p}
$$

A tensão atuante é dada por:

$$
\sigma=E \varepsilon^{e}=E\left(\varepsilon-\varepsilon^{p}\right)
$$

Derivando-se a expressão acima em relação ao tempo temos:

$$
\dot{\sigma}=E \dot{\varepsilon}^{e}=E\left(\dot{\varepsilon}-\dot{\varepsilon}^{p}\right)
$$

onde:

$$
\dot{\varepsilon}^{p}=\frac{d \varepsilon^{p}}{d t} \text { é a taxa de deformação plástica, sendo que as deformações }
$$

permanentes ocorrem apenas quando esta taxa é diferente de zero. A deformação plástica acumulada num certo intervalo de tempo é dada pela integral da taxa neste mesmo intervalo. O tempo, neste caso, representa a história do carregamento, que no problema real é sempre incremental, cargas são aplicadas progressivamente nas estruturas. Representa-se usualmente o valor absoluto da velocidade de deformação plástica por:

$$
\dot{\lambda}=\left|\dot{\varepsilon}^{p}\right|
$$




\subsubsection{MODELO ELASTOPLÁSTICO COM ENCRUAMENTO - CASO UNIAXIAL}

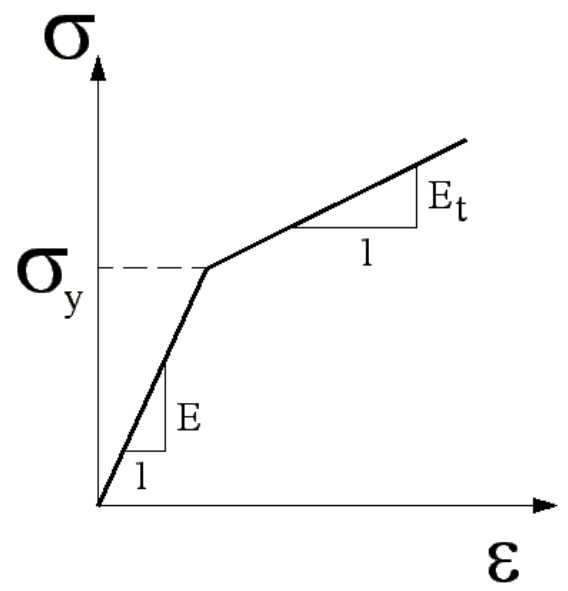

Figura 6.2 - Modelo elastoplástico com encruamento positivo

Neste modelo, conforme figura 6.2, o limite elástico inicial se expande com a evolução da plastificação. Trata-se de modelo adequado para os casos de carregamento crescente, como os casos reais do presente trabalho.

Podemos ter nesse caso duas possibilidades de encruamento: encruamento isótropo e encruamento cinemático.

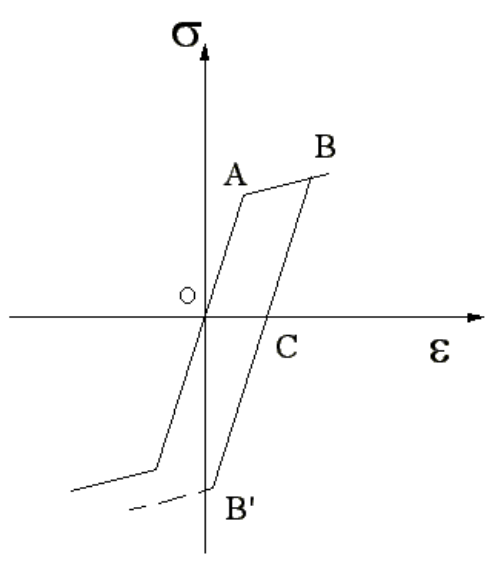

a)

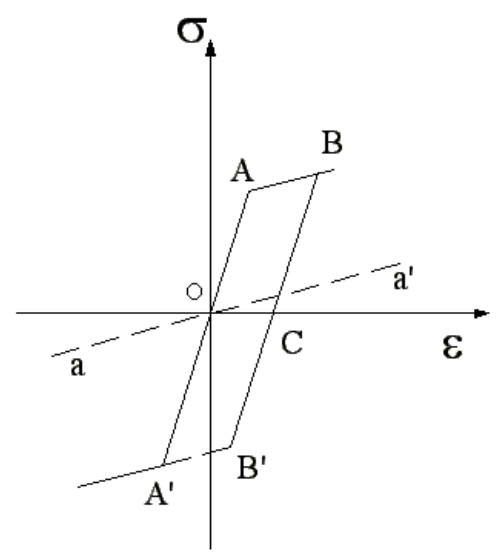

b)

Figura 6.3 - Encruamento isótropo e encruamento cinemático 
No encruamento isótropo, o limite elástico inicial se expande de maneira simétrica em relação ao centro do intervalo inicial, ou seja, o centro do intervalo permanece inalterado mas os limites elásticos de tração e compressão se expandem simetricamente, mantendo-se as condições iniciais de isotropia do material (figura 6.3a). o encruamento cinemático há também evolução dos limites elásticos, porém o centro do intervalo sofre translação (figura 6.3b).

No nosso trabalho será usado o modelo com encruamento isótropo positivo.

O critério de plastificação neste caso é dado por:

$$
f(\sigma, \alpha)=|\sigma|-\left(\sigma_{y}+K \alpha\right)
$$

\section{ONDE:}

$K$ é chamado módulo plástico ou parâmetro de endurecimento, dado pela tangente à curva $\sigma \times \varepsilon^{p}$

$$
\begin{aligned}
& \alpha=\left|\dot{\varepsilon}^{p}\right| \text { é a variável associada ao encruamento } \\
& (\sigma+K \alpha) \text { é o novo limite elástico, depois do encruamento }
\end{aligned}
$$

\subsubsection{MODELO ELASTOPLÁSTICO COM ENCRUAMENTO - CASO MULTIAXIAL}

Neste caso, o critério de plastificação será dado por:

$$
f(\bar{\sigma}, p)=\bar{\sigma}-\sigma_{y}(p) \leq 0
$$

onde:

$\sigma$ é a tensão efetiva equivalente à do estado uniaxial, calculada a partir do critério adotado, neste trabalho o critério de Von Mises. (ver figura 6.4 adiante)

$$
\begin{aligned}
& \bar{\sigma}_{y}(p) \text { é a nova tensão de plastificação, após o encruamento, dada por } \\
& \sigma_{y}(p)=\left(\sigma_{y}-p\right) \\
& p=-K \bar{\varepsilon}^{p}
\end{aligned}
$$


é a variável associada ao encruamento isótropo

$\bar{\varepsilon}^{p}$ é a deformação plástica efetiva, ou seja, a deformação plástica equivalente ao estado uniaxial

$\sigma_{y}$ é a tensão inicial de escoamento

No caso multiaxial, o critério de plastificação será representado por uma superfície de plastificação.

Assim, num carregamento de uma peça estrutural, feito iterativamente, em uma dada iteração $\mathbf{n}+\mathbf{1}$ podemos ter três casos:

Se $\dot{f}<0$ ocorre descarregamento plástico e o ponto está interno à superfície de plastificação.

Se $\dot{f}=0$ podemos ter:

$\dot{\lambda}>0$ tem-se carregamento plástico (o ponto cai fora da superfície de plastificação quando soma-se o incremento de tensão elástico com a tensão verdadeira da iteração anterior) com evolução da deformação plástica e do encruamento.

$\dot{\lambda}=0$ tem-se um carregamento neutro que ocorre em materiais perfeitamente plásticos onde não há evolução da deformação plástica, a superfície de plastificação permanece inalterada e o ponto caminha sobre ela.

O tensor de deformações, analogamente ao caso uniaxial, pode ser decomposto em duas parcelas, uma elástica e uma plástica:

$$
\varepsilon_{i j}=\varepsilon_{i j}^{e}+\varepsilon_{i j}^{p}
$$

Em termos de taxas referidas ao tempo:

$$
\dot{\varepsilon}_{i j}=\dot{\varepsilon}_{i j}^{e}+\dot{\varepsilon}_{i j}^{p}
$$

A lei de plastificação, ou regra de fluxo é dada por:

$$
\dot{\varepsilon}_{i j}^{p}=\dot{\lambda} r(\sigma, \alpha)
$$

Nesta expressão: 
$\dot{\lambda}$ é o chamado multiplicador plástico

$r=\frac{\partial Q}{\partial \sigma_{i j}}$ representa o gradiente de tensão do potencial plástico $\mathbf{Q}$ e estabelece

a direção do fluxo plástico. Quando se considera o potencial plástico $\mathbf{Q}$ coincidente com a função de escoamento $\mathbf{f}$ (equação 6.6), diz-se que a lei de fluxo é associativa.

Na fase elástica, vale a seguinte relação entre os incrementos de tensão e deformação:

$$
d \sigma_{i j}=C_{i j k l} d \varepsilon_{k l}^{e}
$$

onde $C_{i j k l}$ é o tensor elástico dos materiais isótropos.

Na fase plástica, o caso multiaxial é associado ao caso uniaxial através da tensão e da deformação plástica efetivas. A relação elastoplástica entre tensão e deformação é dada por:

$$
\sigma_{i j}=C_{i j k l}\left(\varepsilon_{k l}-\varepsilon_{k l}^{p}\right)=C_{i j k l}^{e p} \varepsilon_{k l}
$$

onde $C^{e p}$ é o tensor dos módulos elastoplasticos tangentes.

Derivando-se a expressão (6.6), considerando-se a lei de consistência e substituindo na expressão (6.11) temos:

$$
\begin{aligned}
& \dot{\lambda}=\frac{f_{\sigma} \bullet C \dot{\varepsilon}}{f_{\sigma} \bullet C r+f_{p} h} \\
& C^{e p}=\left\{\begin{array}{l}
C \quad \text { se } \dot{\lambda}=0 \\
C-\frac{\left(C r \otimes C f_{\sigma}\right)}{f_{\sigma} C r+f_{p} h} \text { se } \quad \dot{\lambda}>0
\end{array}\right.
\end{aligned}
$$

No caso da lei de fluxo associativa, o tensor da taxa de deformação plástica passa a ter a direção normal à superfície de plastificação, ou seja:

$$
\dot{\varepsilon}^{p}=\dot{\lambda} f_{\sigma}
$$

A partir daí pode ser deduzido que: 


$$
\begin{aligned}
& \dot{\lambda}=\frac{f_{\sigma} C \dot{\varepsilon}}{f_{\sigma} C f_{\sigma}+K} \\
& C^{e p}=\left\{\begin{array}{l}
C \quad \text { se } \quad \dot{\lambda}=0 \\
C-\frac{\left(C f_{\sigma} \otimes C f_{\sigma}\right)}{f_{\sigma} C f_{\sigma}+K} \text { se } \quad \dot{\lambda}>0
\end{array}\right.
\end{aligned}
$$

Considerando um algorítmo implícito no cálculo das tensões, bem como o critério de plastificação de Von Mises, a lei da normalidade e o encruamento isótropo positivo podemos ter uma interpretação geométrica do problema, conforme figura abaixo. No caso de algoritmo implícito, a correção do estado de tensões, quando o ponto que as representa está fora da superfície de plastificação, é feita segundo a direção da normal à superfície de plastificação na posição atual $(\mathbf{n}+\mathbf{1})$ enquanto que num algoritmo implícito, a mesma seria feita segundo a normal à superfície da iteração anterior (n).

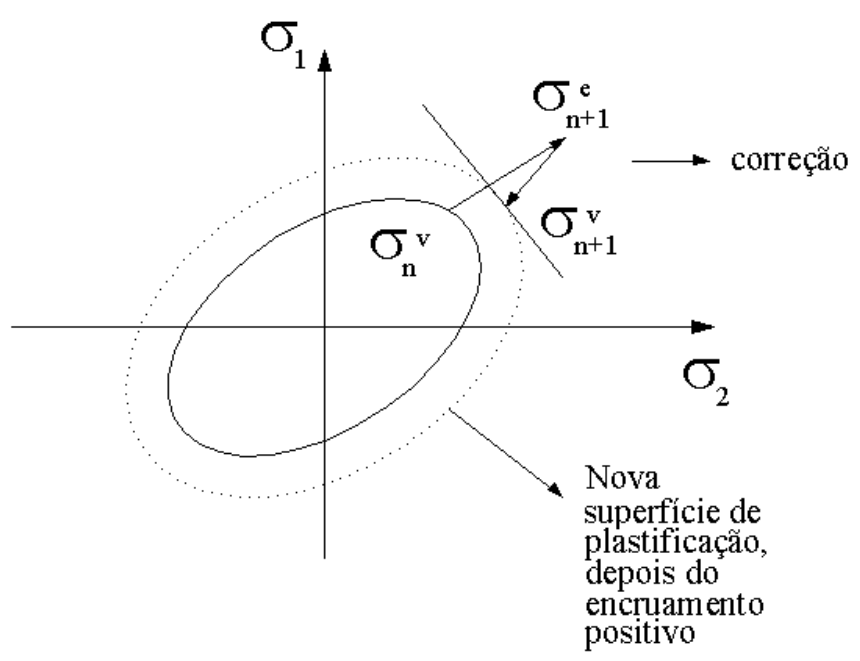

Figura 6.4 - Representação geométrica do critério de Von Mises no caso biaxial Escrevendo as relações anteriores em notação indicial e considerando-se:

$$
f_{\sigma}=\frac{\partial f\left(\sigma_{i j}\right)}{\partial \sigma_{i j}}=\frac{\partial \bar{\sigma}}{\partial \sigma_{i j}}=a_{i j}
$$


$d_{i j}=a_{i j} C_{i j k l}$

temos:

$\dot{\varepsilon}_{i j}^{p}=\dot{\lambda} a_{i j}$

$\dot{\lambda}=\frac{a_{i j} C_{i j k l} \dot{\varepsilon}_{k l}}{a_{i j} d_{i j}+}=\frac{a_{i j} \dot{\sigma}_{i j}}{a_{i j} d_{i j}+K}$

sendo $\bar{\sigma}_{i j}$ o incremento de tensão acima do limite elástico.

$C^{e p}=\left\{\begin{array}{l}C_{i j k l} \quad \text { se } \quad \dot{\lambda}=0 \\ C_{i j k l}-\frac{\left(d_{i j} a_{m n} C_{m n k l}\right)}{a_{m n} d_{m n}+} \text { se } \quad \dot{\lambda}>0\end{array}\right.$.

Desta forma, o incremento verdadeiro de tensão é dado por

$$
d \sigma_{i j}^{v}=d \sigma_{i j}^{e}-d \sigma_{i j}^{p}
$$

onde:

$$
\begin{aligned}
& d \sigma_{i j}^{e}=C_{i j k l} d \varepsilon_{k l} \\
& d \sigma_{i j}^{p}=\frac{d_{i j} a_{m n}}{a_{m n} d_{m n}+K} d \sigma_{m n}^{e}=d_{i j} d \lambda
\end{aligned}
$$

A lei de evolução do encruamento é dada em função do trabalho plástico realizado pelas deformações, segundo a hipótese de “work hardening”, ou seja:

$$
W_{p}=\int \sigma_{i j} d \varepsilon_{i j}^{p}=\bar{\sigma}_{(n+1)}^{p} \Delta \bar{\varepsilon}_{(n+1)}^{p}=\sigma_{i j(n+1)}^{v} \Delta \varepsilon_{i j(n+1)}^{p}
$$

onde os valores com o traço superior são os efetivos.

Escrevendo a equação (6.18) em termos de incrementos e substituindo-a em (6.24) obtém-se o incremento de deformação plástica efetiva: 


$$
\Delta \bar{\varepsilon}_{n+1}^{p}=\frac{\Delta \lambda a_{i j} \sigma_{i j(n+1)}^{v}}{\bar{\sigma}_{n+1}^{v}}
$$

Assim, o roteiro para se obter a tensão verdadeira em uma determinada iteração $(\mathbf{n}+\mathbf{1})$ é dado por:

Supõe-se que a iteração seja elástica:

$$
\begin{aligned}
& \varepsilon_{i j(n+1)}=\varepsilon_{i j(n)}+\Delta \varepsilon_{i j(n+1)}^{e} \\
& \sigma_{i j(n+1)}^{e}=E\left(\varepsilon_{i j(n+1)}-\varepsilon_{i j(n)}^{p}\right)=\sigma_{i j(n)}^{v}+\Delta \sigma_{i j(n+1)}^{e} \\
& \bar{\varepsilon}_{n+1}^{p}=\bar{\varepsilon}_{n}^{p}
\end{aligned}
$$

Com $\sigma_{i j(n+1)}^{e}$ calcula-se $\bar{\sigma}_{(n+1)}^{e}$ de acordo com o critério especificado.

Verifica-se o critério de plastificação:

$$
f_{n+1}=\bar{\sigma}_{n+1}^{e}-\left(\sigma_{y}+K \bar{\varepsilon}_{n+1}^{p}\right) \leq 0
$$

Deve ser obedecida a condição: $\Delta \lambda f_{n+1}=0$

Se $f_{n+1} \leq 0$ temos $\Delta \lambda=0$ e portanto $\sigma_{i j(n+1)}^{v}=\sigma_{i j(n+1)}^{e}$

Se $f_{n+1}>0$ temos $\Delta \lambda>0$ e devemos procurar um novo estado de tensão tal que $f_{n+1}=0$ como mostrado na figura (6.4). Calcula-se então o novo valor de $\Delta \lambda$, o incremento de deformação plástica pela equação (6.25), atualiza-se o valor de $\varepsilon_{n+1}^{p}=\varepsilon_{n}^{p}+\Delta \varepsilon_{n+1}^{p}$ e calcula-se a tensão verdadeira:

$$
\sigma_{i j(n+1)}^{v}=\sigma_{i j(n)}^{v}+\Delta \sigma_{i j(n+1)}^{v}=\sigma_{i j(n)}^{v}+\Delta \sigma_{i j}^{e}-\Delta \lambda d_{i j}
$$

\subsubsection{CRITÉRIO DE PLASTIFICAÇÃO DE VON MISES}

Um critério de plastificação deve sempre ser independente do sistema de coordenadas empregado, sendo habitualmente dado em função dos invariantes de tensão. No critério proposto por Von Mises, o escoamento acontece quando o segundo invariante de tensão atinge um valor $\mathbf{k}$, ou seja”: 
$\sqrt{J_{2}}=k$

onde $J_{2}=\frac{1}{2} S_{i j} S_{i j} \quad(i, j=1,2,3)$

sendo $S_{i j}$ a parte anti-esférica do vetor de tensões que é dado por:

$S_{i j}=\sigma_{i j}-\delta_{i j} \sigma_{m}$

onde $\sigma_{m}=\frac{\sigma_{i j}}{3}$

Podemos escrever a equação (6.28) em função das tensões principais:

$$
J_{2}=\frac{1}{6}\left[\left(\sigma_{1}-\sigma_{2}\right)^{2}+\left(\sigma_{2}-\sigma_{3}\right)^{2}+\left(\sigma_{3}-\sigma_{1}\right)^{2}\right]
$$

Considerando o caso bidimensional em que a tensão na direção três é nula temos:

$$
\sigma_{1}^{2}+\sigma_{2}^{2}-\sigma_{1} \sigma_{2} \leq 3 k^{2}
$$

que é representada por uma elipse conforme figura (6.4)

O critério passa então a ser:

$$
f(\bar{\sigma}, p)=\sqrt{3 J_{2}}-\sigma_{y}(p)=0
$$

A tensão efetiva será então dada por:

$$
\bar{\sigma}=\sqrt{3 J_{2}}
$$

Escrevendo agora a equação (6.28) para o sistema de coordenadas $\mathbf{x}_{1} \mathbf{x}_{\mathbf{2}}$ :

$$
J_{2}=\sigma_{12}^{2}+\frac{1}{2}\left[\left(\sigma_{11}-\sigma_{m}\right)^{2}+\left(\sigma_{22}-\sigma_{m}\right)^{2}+\sigma_{m}^{2}\right] .
$$

Desse modo os valores de $\mathbf{a}_{\mathbf{i j}}$ dados na equação (6.16) resultam em: 


$$
a_{i j}=\frac{\partial f}{\partial J_{2}} \frac{\partial J_{2}}{\partial \sigma_{i j}}=\frac{\sqrt{3}}{2 \sqrt{J_{2}}} \frac{\partial J_{2}}{\partial \sigma_{i j}} .
$$

\subsection{MECÂNICA DO DANO}

\subsubsection{INTRODUÇÃO}

Apresentam-se aqui, de forma sucinta, aspectos básicos da mecânica do dano contínuo, aplicado ao concreto, bem como o modelo de dano de Mazars, aqui utilizado. Maiores detalhes podem ser encontrados nos trabalhos de MAZARS (1984), PEREGO(1990), ÁLVARES(1993), BUSSAMRA(1993). No Departamento de Engenharia de Estruturas da EESC foram desenvolvidas algumas dissertações e teses onde se focalizou algumas aplicações de modelos de dano às estruturas de concreto armado. Podem ser citados: BOTTA(1998), PITUBA(1998) e DRIEMEIER (1999).

Os modelos fundamentados na teoria da mecânica do dano, nos meios contínuos, lidam com a previsão do comportamento mecânico para sólidos com micro-defeitos (pequenas fissuras e vazios) em seu arranjo estrutural interno, sem grandes fissuras que caracterizam o campo da mecânica da fratura.

Algumas definições básicas, de acordo com LEMAITRE \& CHABOCHE (1985) propiciaram o aperfeiçoamento de metodologias próprias para formular modelos de dano. No caso de modelos isotrópicos, são importantes as definições de elemento representativo de volume, de variável escalar de dano, definida a partir das áreas resistente e total de uma seção que passa pelo elemento de volume, de tensão efetiva, do princípio de deformação equivalente e de como efetuar medidas indiretas de dano em laboratório. Além disso, o enquadramento das formulações matemáticas dos modelos no formalismo da termodinâmica dos processos irreversíveis, propicia sentido físico à representação do processo de danificação, respeitando os processos energéticos envolvidos.

Posto que na termodinâmica clássica os sistemas são estudados através de equações de estado, onde as hipóteses são a reversibilidade dos processos e que a 
danificação dos materiais sólidos é um processo claramente irreversível, faz-se necessária a generalização dos conceitos da termodinâmica para que seja, assim, excluída a reversibilidade.

\subsubsection{VARIÁVEL DE DANO}

Seja um elemento de volume representativo de um sólido danificado. Este elemento é considerado suficientemente pequeno de forma que possa ser interpretado como um ponto material do contínuo, ao mesmo tempo suficientemente grande para conter as imperfeições e representar o material constitutivo do meio.

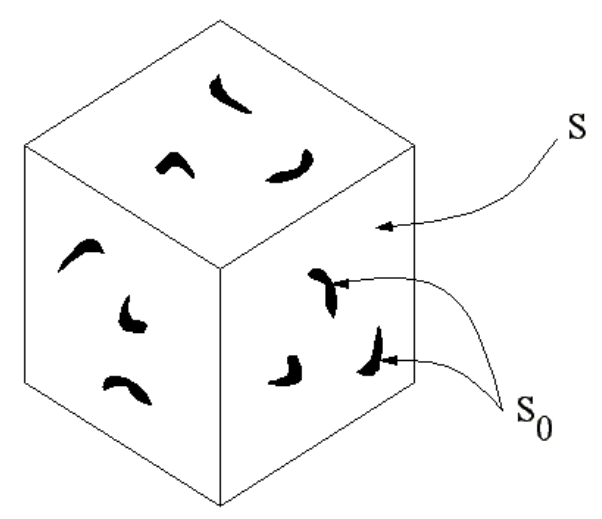

Figura 6.5 - Elemento de volume danificado

Na figura (6.5) temos:

$S$ é a área total de uma seção deste elemento

$S_{0}$ é a soma total das áreas dos defeitos desta superfície

$\bar{S}$ é a área resistente efetiva, descontados os defeitos

Vale a seguinte relação:

$$
S_{0}=S-\bar{S}
$$

Uma medida do dano local, admitindo-se a hipótese de homogeneidade, bem como a isotropia do material é dada por: 


$$
D=\lim _{S \rightarrow 0} \frac{S}{S_{0}}
$$

A variável de dano D é portanto um escalar no caso considerado.

\subsubsection{DEFORMAÇÃO EQUIVALENTE}

Seja um corpo de prova de área de seção $\mathbf{S}$ submetido à tração de uma força $\mathbf{F}$ conforme figura abaixo.

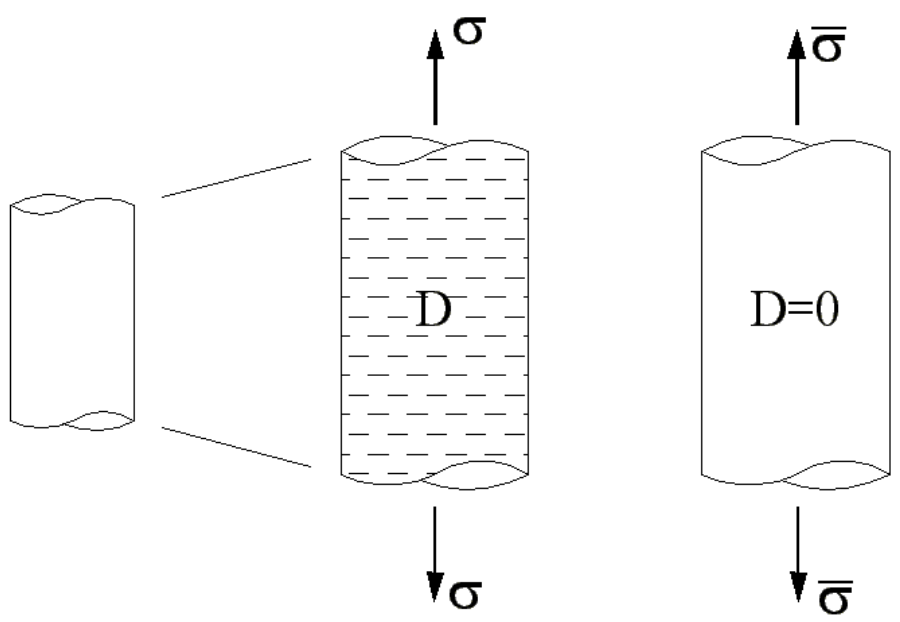

Figura 6.6 - Deformação equivalente

Temos que:

$D=\frac{S_{0}}{S}$

Considerando as equações (6.39) e (6.37) temos:

$\bar{S}=S(1-D)$

Temos ainda que a tensão de tração aplicada vale:

$\sigma=\frac{F}{S}$

A tensão efetiva, aplicada à parte resistente da seção fica: 


$$
\bar{\sigma}=\frac{\sigma}{\bar{S}}=\frac{\sigma S}{S(1-D)}=\frac{\sigma}{(1-D)}
$$

onde os casos de:

$\bar{\sigma}=\sigma$ representa o material íntegro

$\bar{\sigma} \rightarrow \infty$ representa o estado limite de deterioração

A deformação elástica no material com dano pode ser descrita em função da deformação do material íntegro da seguinte forma:

$$
\varepsilon=\frac{\bar{\sigma}}{E}=\frac{\sigma}{E(1-D)}=\frac{\sigma}{\bar{E}}
$$

onde $\bar{E}=E(1-D)$ é o módulo de elasticidade do material danificado

\subsubsection{MODELO DE DANO DE MAZARS}

Apresenta-se a seguir o modelo de dano proposto por MAZARS(1984). Este modelo, extremamente simples, é apropriado para o concreto submetido a um carregamento proporcional crescente, utilizado no presente trabalho.

\subsubsection{HIPÓTESES BÁSICAS}

As suas hipóteses são:

- $\quad$ Em processo de dano evolutivo, o concreto apresenta comportamento elástico, desprezando-se portanto as deformações permanentes observadas numa situação de descarregamento;

- $\quad$ O dano é causado somente pela existência de alongamentos que ocorrerão ao longo das direções principais de deformação;

- $\quad$ O dano é isótropo, ou seja, o estado de dano num ponto é representado por um valor escalar.

Representa-se o dano local pela variável escalar D que varia entre 0 e 1 e cuja evolução ocorre quando for superado um valor de referência para o alongamento equivalente. 


\subsubsection{DEFORMAÇÕES PRINCIPAIS}

A relação tensão-deformação é dada pela Lei de Hooke e podem ser expressas da seguinte forma em função de suas partes positivas e negativas:

$$
\sigma_{i}=\sigma_{i}^{+}+\sigma_{i}^{-}
$$

onde:

$$
\text { se } \sigma_{i}>0 \quad \sigma_{i}^{+}=\sigma_{i} \quad \sigma_{i}^{-}=0
$$

se $\sigma_{i}<0 \quad \sigma_{i}^{+}=0 \quad \sigma_{i}^{-}=\sigma_{i}$

Assim, as deformações devidas às tensões de tração podem ser expressas separadamente das deformações devidas às tensões de compressão, obtidas pela lei de Hooke e dadas por:

$$
\begin{aligned}
& \varepsilon_{T i}=\frac{1+v}{E} \sigma_{i}^{+}-\frac{v}{E} \sum_{i=1}^{3} \sigma_{i}^{+} \\
& \varepsilon_{C i}=\frac{1+v}{E} \sigma_{i}^{-}-\frac{v}{E} \sum_{i=1}^{3} \sigma_{i}^{-}
\end{aligned}
$$

A deformação total em cada direção principal fica dada por:

$$
\varepsilon_{i}=\varepsilon_{T i}+\varepsilon_{C i}
$$

\subsubsection{DEFORMAÇÃO EQUIVALENTE}

Os alongamentos referentes a cada direção principal são:

$$
\varepsilon_{i}^{+}=\frac{1}{2}\left(\varepsilon_{i}+\left|\varepsilon_{i}\right|\right)
$$

onde:

$$
\begin{array}{llll}
\varepsilon_{i}^{+}=\varepsilon_{i} & \text { se } & \varepsilon_{i}>0 \\
\varepsilon_{i}^{+}=0 & \text { se } & \varepsilon_{i} \leq 0
\end{array}
$$

Com os valores dos deslocamentos calculados acima, a deformação equivalente, que traduz o estado do material no ponto é dada por: 


$$
\bar{\varepsilon}=\sqrt{\left(\varepsilon_{1}^{+}\right)^{2}+\left(\varepsilon_{2}^{+}\right)^{2}+\left(\varepsilon_{3}^{+}\right)^{2}}
$$

\subsubsection{CRITÉRIO DE DANO}

Admite-se que o processo de danificação do material tem início quando a deformação equivalente atinge um valor limite, dada pelo valor da deformação correspondente no ensaio uniaxial de tração, ao valor da máxima tensão, como ilustra a figura abaixo.

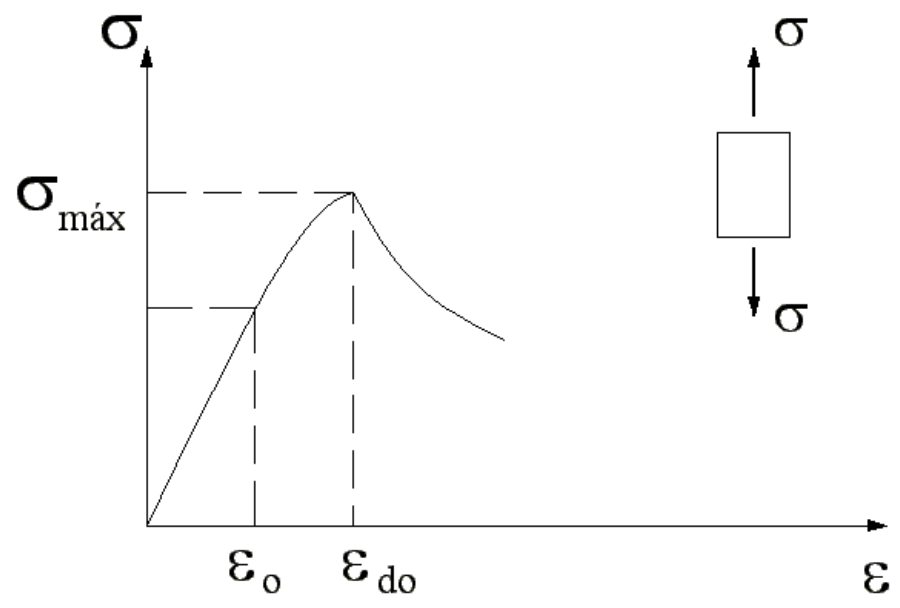

Figura 6.7 - Representação de $\varepsilon_{d 0}$

Assim, o critério de dano fica expresso por:

$$
f(\bar{\varepsilon}, D)=\bar{\varepsilon}-S(D) \leq 0
$$

onde:

$S_{0}=S(D=0)=\varepsilon_{d 0}$ é o limite elástico inicial.

Fazendo-se $f(\bar{\varepsilon}, D)=0$ e substituindo na equação (6.47) temos:

$$
S(D)=\bar{\varepsilon}=\sqrt{\left(\varepsilon_{1}^{+}\right)^{2}+\left(\varepsilon_{2}^{+}\right)^{2}+\left(\varepsilon_{3}^{+}\right)^{2}}
$$


Considerando o espaço das tensões principais, a função $\mathbf{S}(\mathbf{D})$ representa uma superfície de um oitavo de esfera de raio $\mathbf{S}(\mathbf{D})$, oitavo este onde todas as tensões são positivas.

Um ponto dentro da superfície significa que o estado de alongamento equivalente é menor que o limite elástico, não havendo evolução do dano. Um ponto fora da superfície caracteriza a evolução do dano.

A lei de evolução do dano $\mathbf{D}$ que atende aos princípios da termodinâmica dos processos irreversíveis é dada por:

$$
\begin{aligned}
& \dot{D}=0 \quad \text { se } \quad f(\bar{\varepsilon}, D) \leq 0 \quad e \quad \dot{f}(\bar{\varepsilon}, D)<0 \text { (caso de descarregamento) } \\
& \dot{D}>0 \text { se } f(\bar{\varepsilon}, D)=0 \quad e \quad \dot{f}(\bar{\varepsilon}, D)=0 \text { (caso de carregamento) } \\
& \dot{D}=0 \quad e \quad \dot{f}(\bar{\varepsilon}, D)=0 \text { (carregamento neutro) }
\end{aligned}
$$

Não se admite (f $>\mathbf{0})$, quando isto ocorre, há evolução da variável de dano e o novo limite elástico passa a ser $\mathbf{S}(\mathbf{D})$, definido pelo estado de alongamento atual.

\subsubsection{DETERMINAÇÃO DA VARIÁVEL DE DANO}

Dado o comportamento não simétrico do concreto à tração e à compressão uniaxiais, ocorre uma diferença no processo de evolução da microfissuração. Enquanto que em tração as microfissuras se desenvolvem perpendicularmente à direção do esforço, em compressão elas se desenvolvem paralelamente ao esforço. Isto leva a definição de duas variáveis escalares de dano, uma ligada a cada tipo de fissura, de tração ou compressão. O processo de evolução de dano para estes dois casos é governado por leis independentes.

Como no caso multiaxial, cada componente de tração pode contribuir para a evolução da danificação do material, a variável de dano fica determinada por combinação linear destes valores referentes à tração e à compressão uniaxiais, para o caso de carregamento proporcional. Logo:

$$
\begin{aligned}
& D=\alpha_{T} D_{T}+\alpha_{C} D_{C} \\
& 0 \leq \alpha_{T} \leq 1 \quad 0 \leq \alpha_{C} \leq 1 \quad \alpha_{T}+\alpha_{C}=1
\end{aligned}
$$


As expressões para estes valores são dadas por:

$$
\begin{aligned}
& D_{T}(\bar{\varepsilon})=1-\frac{\varepsilon_{d 0}\left(1-A_{T}\right)}{\bar{\varepsilon}}-\frac{A_{T}}{\exp \left[B_{T}\left(\bar{\varepsilon}-\varepsilon_{d 0}\right)\right]} \ldots \ldots \ldots \ldots \ldots \ldots \ldots \ldots \ldots \ldots \ldots \\
& D_{C}(\bar{\varepsilon})=1-\frac{\varepsilon_{d 0}\left(1-A_{C}\right)}{\bar{\varepsilon}}-\frac{A_{C}}{\exp \left[B_{T}\left(\bar{\varepsilon}-\varepsilon_{d 0}\right)\right]} \\
& \alpha_{T}=\frac{\sum_{i=1}^{3} \varepsilon_{T i}^{+}}{\varepsilon_{v}^{+}} ; \quad \alpha_{C}=\frac{\sum_{i=1}^{3} \varepsilon_{C i}^{+}}{\varepsilon_{v}^{+}} ; \varepsilon_{v}^{+}=\sum_{i=1}^{3}\left(\varepsilon_{T i}^{+}+\varepsilon_{C i}^{+}\right) \ldots
\end{aligned}
$$

sendo $\varepsilon_{T}^{+}$e $\varepsilon_{C}^{+}$as componentes positivas dos tensores $\varepsilon_{T}$ e $\varepsilon_{C}$ obtidos a partir das equações (6.44), $\varepsilon_{T}^{+}$e $\varepsilon_{C}^{+}$são determinados considerando-se a equação (6.46), os valores de $A_{T}, A_{C}, B_{T}, B_{C}$ são parâmetros característicos do material. Estes parâmetros foram estudados por ALVARES(1993) que determinou seus valores para determinados tipos de concreto. 


\section{SOLUÇÃO DO PROBLEMA NÃO LINEAR}

\subsection{INTRODUÇÃO}

Será considerado neste trabalho apenas a não linearidade física do concreto, representada pelos modelos constitutivos estudados no capítulo 6, não será considerada a não linearidade geométrica do problema.

A solução do problema de não linearidade física foi inicialmente analisada por ZIENKIEWICS et al.(1969), que desenvolveu um processo denominado de processo das tensões iniciais, empregado com o Método dos Elementos Finitos.

Trata-se de um procedimento incremental, onde o carregamento total é dividido em parcelas (incrementos) de carga, aplicados um a um. O procedimento de busca do equilíbrio da estrutura a cada incremento de carga é iterativo, obtido após várias iterações até chegar-se a uma margem de erro previamente estabelecida (tolerância). O processo deve convergir dentro de um limite máximo de iterações estabelecido, se tal não ocorrer a estrutura é considerada como não mais capaz de encontrar um estado de equilíbrio, isto é, chegou ao seu limite.

O trabalho inicial neste sentido foi realizado por OWEN \& HINTON (1980), adaptado para o MEC por RIBEIRO(1992). 


\subsection{MODELO ADOTADO}

Em trabalhos anteriores na EESC, FERNANDES(1998) e BACARJI(2000) trataram do problema considerando a placa dividida em camadas de espessuras e propriedades diferentes, constituindo o chamado modelo estratificado.

O presente trabalho leva em conta que, no caso real do concreto armado, temos apenas dois materiais distintos, o concreto e o aço, para os quais são adotados os modelos constitutivos já considerados. Na seção de concreto, admitida com características físicas uniformes, são distribuídos pontos de Gauss (8,10 ou 12) e na posição de cada uma das armaduras positiva ou negativa é também colocado um ponto representativo.

A distribuição das deformações ao longo da espessura é linear, mesmo se o estado de tensão tenha atingido um comportamento não-linear. Não há necessidade de se definir a forma da distribuição de tensões em todos os pontos, pois como a integração é feita através da fórmula da quadratura de Gauss, as propriedades do material e o valor das tensões precisam ser especificados apenas nestes pontos.

O problema não-linear é resolvido sempre, na primeira vez, para o valor total do carregamento, admitindo comportamento elástico linear. Os momentos são então calculados para os pontos internos da placa, vértices das células internas situados fora do contorno, bem como para os pontos internos adicionais correspondentes aos vértices das células situadas no contorno.

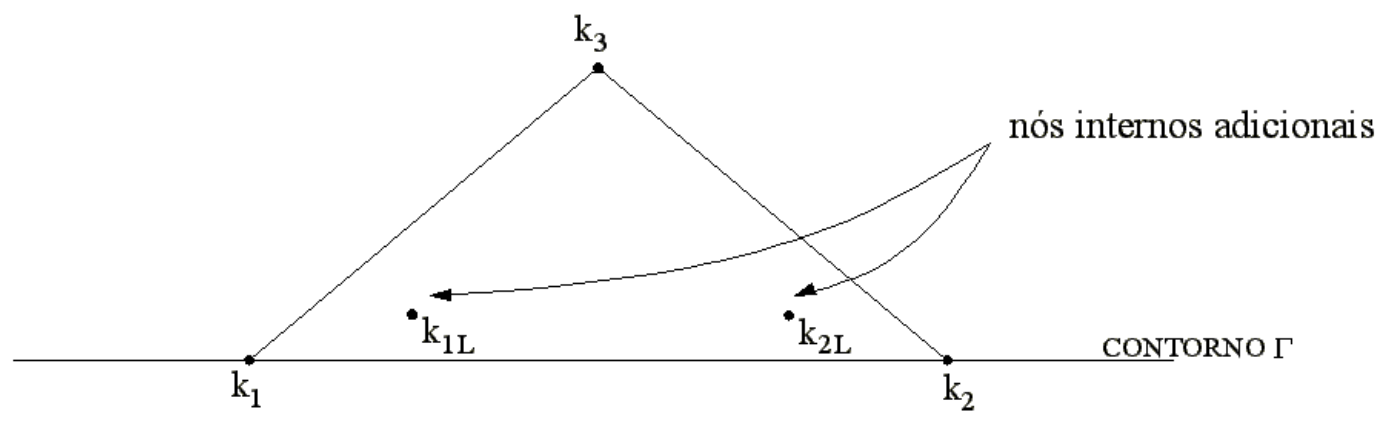

Figura 7.1 - Pontos onde são calculados os momentos internos 
Obtém-se então o vetor de momentos elásticos totais $\mathbf{M}^{e}$ para o carregamento atuante, nos pontos considerados.

\subsection{CÁLCULO DO MOMENTO INTERNO RESULTANTE NUMA SEÇÃO DA PLACA}

Sendo $\mathbf{t}$ a espessura da placa e $\mathbf{x}_{\mathbf{3}}$ a coordenada correspondente a ela temos:

$$
-t / 2 \leq x_{3} \leq t / 2
$$

Definindo uma coordenada homogênea $\xi$ nesta mesma direção, variando entre -1 e 1 para a espessura da placa temos:

$$
\begin{aligned}
& x_{3}=\xi \frac{t}{2} \\
& d x_{3}=\frac{t}{2} d \xi
\end{aligned}
$$

Os pontos de Gauss, definidos ao longo da espessura em função da coordenada homogênea representarão a seção de concreto enquanto que as armaduras serão distribuídas em pontos adicionais cujas posições são previamente estabelecidas.

A partir desta aproximação, os momentos resultantes na seção são obtidos pela integração das respectivas componentes de tensão ao longo da espessura:

$$
M_{i j}=\int_{-t / 2}^{t / 2} \sigma_{i j}^{C} x_{3} d x_{3}+\sum_{n=1}^{N s} \sigma_{i j}^{S(n)} \delta_{i j} A_{s(n)} x_{3 S}^{n} \quad(i, j=1,2)
$$

onde:

$\sigma_{i j}^{C}$ é a tensão na placa de concreto

Ns é o número de armaduras

$x_{3 S}^{n}$ é a posição da armadura considerada

$\sigma_{i j}^{S(n)}$ é a tensão na armadura n

$A_{S(n)}$ é a área da armadura n 
Fazendo a mudança de coordenadas para se obter a integração numérica, podemos escrever a equação (7.2) como:

$$
M_{i j}=\frac{t^{2}}{4} \sum_{i g=1}^{N g} \sigma_{i j}^{C(i g)} \xi_{i g} W_{i g}+\sum_{n=1}^{N s} \sigma_{i j}^{S(n)} \delta_{i j} A_{S(n)} x_{3 S}^{n}
$$

onde Ng é o número de pontos de Gauss adotado.

\subsection{EQUILÍBRIO NA SEÇÃO DA PLACA}

A placa é calculada desprezando-se o efeito de membrana, tendo portanto apenas flexão. O valor da força normal atuante em qualquer seção deve ser nulo.

Aplicando-se numa seção o momento dado pela equação (7.3), estamos admitindo que a linha neutra, na seção considerada passa pelo seu ponto médio, isto é admite-se que a distribuição das tensões é simétrica na tração e na compressão. Tal fato não ocorre, além do fato de as armaduras não serem, em geral, simetricamente distribuídas. Com isso, a linha neutra não mais coincide com a superfície média da placa. Como no caso da flexão simples a placa não pode ser submetida a forças normais, deve-se procurar a nova posição da linha neutra para que se tenha a força normal resultante nula (dentro do limite de tolerância).

O comportamento não linear e não simétrico faz com que uma mudança da posição da linha neutra em uma determinada direção influencie o valor da normal resultante em outra direção. Portanto, as posições da linha neutra nas três direções são dependentes e conseqüentemente, cada vez que se estima a posição da mesma numa direção, deve-se verificar a normal resultante nas outras direções. Assim, primeiro fazse nula a resultante da normal na direção $\mathbf{x}_{1}$, calcula-se a linha neutra em $\mathbf{x}_{2}$ e verificase a normal na direção $\mathbf{x}_{\mathbf{1}}$. Este processo continua até que a normal resultante seja nula nas duas direções. Em seguida, estima-se a posição da linha neutra em $\mathbf{x}_{\mathbf{1}} \mathbf{x}_{\mathbf{2}}$ e verificase as direções $\mathbf{x}_{\mathbf{1}}$ e $\mathbf{x}_{\mathbf{2}}$. No final deste processo iterativo, ter-se-á a normal nula nas três direções. 
A estimativa da nova posição da linha neutra, em uma direção ij é feita por interpolação linear usando-se os valores previamente calculados da normal e suas respectivas posições.

$$
Z_{i j}=Z_{i j}^{2}+\frac{N_{i j}^{2}\left(Z_{i j}^{1}-Z_{i j}^{2}\right)}{N_{i j}^{2}-N_{i j}^{1}}
$$

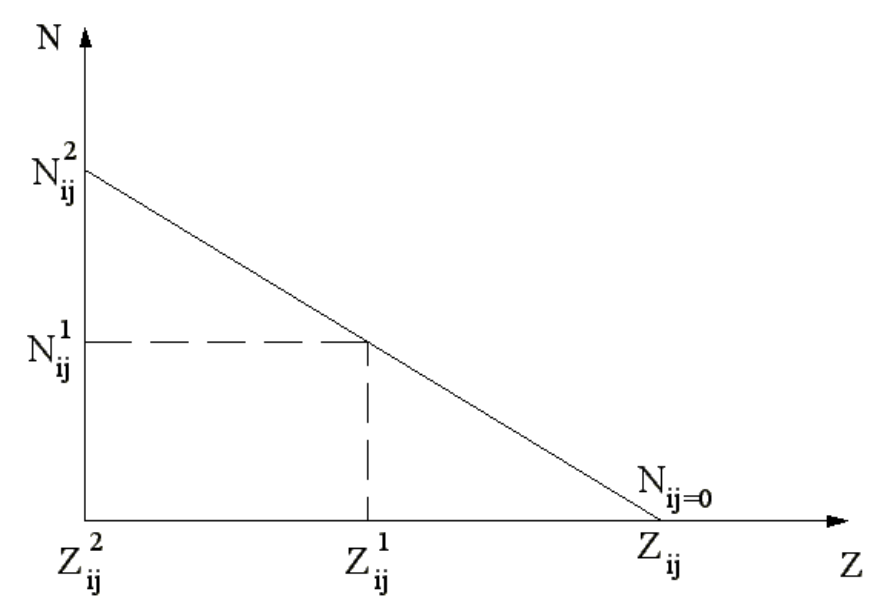

Figura 7.2 - Estimativa da linha neutra

Na primeira estimativa, não há um ponto 2, então adota-se o mesmo de tal forma que a seção fique totalmente comprimida. Assim, calcula-se a normal para esta posição e então faz-se a primeira estimativa de $\mathrm{Z}$ calculando-se a normal $\mathrm{N}$ para esta posição. $\mathrm{A}$ partir da segunda estimativa, teremos três valores da normal, dois da iteração anterior e o agora calculado. Na interpolação seguinte temos:

$$
\begin{aligned}
& N_{i j}^{2}=N_{i j} \\
& N_{i j}^{1}=N_{i j}^{\prime 2} \text { se } \quad\left|Z_{i j}^{\prime 2}-Z_{i j}\right|<\left|Z_{i j}^{\prime 1}-Z_{i j}\right| \text { ou. } \\
& N_{i j}^{1}=N_{i j}^{\prime} \quad \text { em caso contrário }
\end{aligned}
$$


onde: $N_{i j}^{\prime 1} \quad e \quad N_{i j}^{\prime 2}$ vem da iteração anterior

A cada nova estimativa da linha neutra, deve-se calcular o incremento de deformações e de tensões devido à mudança de posição da mesma.

$$
\Delta \varepsilon_{i j}^{L N}=\left(Z_{i j}^{0}-Z i j\right) w_{, i j} \quad(i, j=1,2)
$$

onde:

$Z_{i j}^{0}$ é a posição da linha neutra no início da iteração e $w_{, i j}$ é o vetor das curvaturas.

Somando-se $\Delta \varepsilon_{i j}^{L N}$ ao incremento de deformações elástico $\Delta \varepsilon_{i j}^{e}$ devido ao carregamento, obtém-se o incremento de deformação total $\Delta \varepsilon_{i j}^{T}$. Somando-se este ao vetor de deformações verdadeiras da iteração anterior $\Delta \varepsilon_{i j}^{n-1}$ obtém-se o vetor de deformações totais $\varepsilon_{i j}^{n}$ como mostrado na figura abaixo, para uma direção ij.

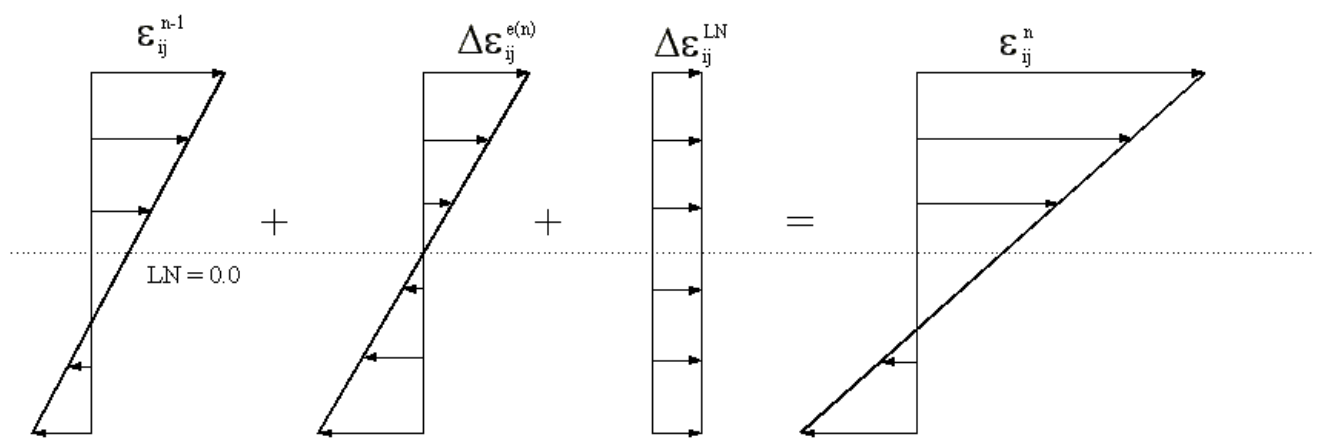

Figura 7.3 - Distribuição das deformações em uma seção da placa

A fim de se determinar a posição da linha neutra $Z_{i j}^{0}$ na direção ij e no início de uma nova iteração n, considere a figura abaixo onde o ponto $\mathrm{P}$ representa um ponto de Gauss qualquer. Sua posição em relação à linha neutra atual é dada por: 


$$
Z_{i j}^{0(P)}=\frac{\varepsilon_{i j}^{n}}{w_{, i j}^{n}}
$$

e em relação á linha neutra situada no meio da seção é dada por:

$$
Z^{P}=\frac{t}{2} \xi_{i g}
$$

A partir daí temos:

$$
Z_{i j}^{0}=Z^{P}-Z_{i j}^{o(P)}
$$

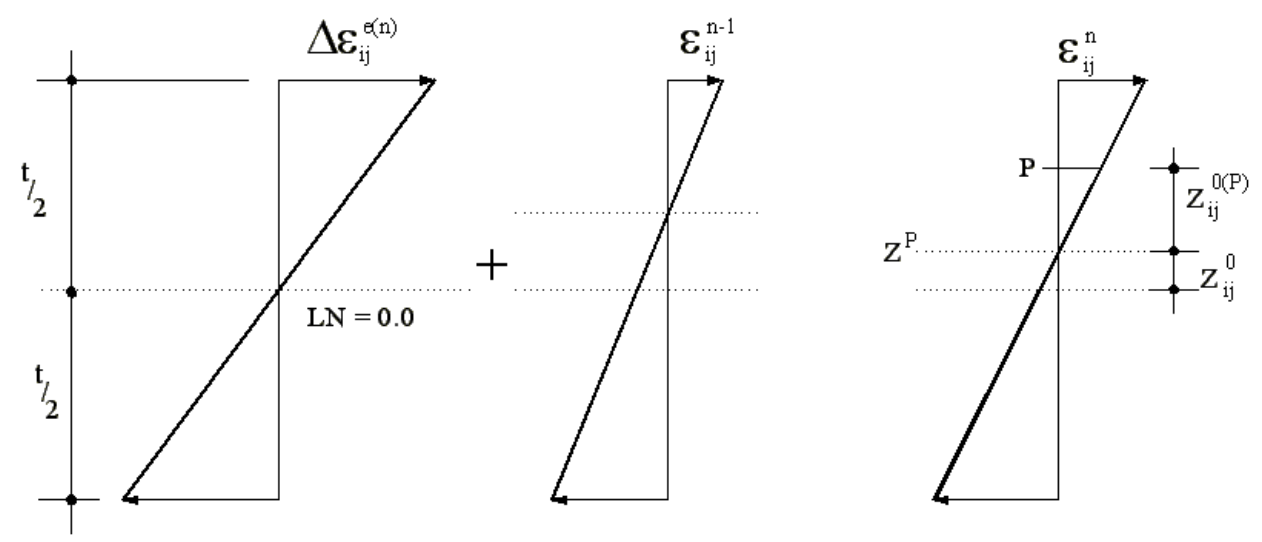

Figura 7.4 Distribuição de deformação resultante em uma seção

Com o incremento de deformações $\Delta \varepsilon_{i j}^{L N}$ calcula-se o incremento de tensões $\Delta \sigma_{i j}^{L N}$ na placa de concreto. Soma-se $\Delta \sigma_{i j}^{L N}$ aos incrementos elásticos de tensão devido ao carregamento $\Delta \sigma_{i j}^{e(n)}$ da iteração em consideração, obtendo-se o incremento de tensão total $\Delta \sigma_{i j}^{T}$. Somando-se esse último às tensões verdadeiras da iteração anterior $\sigma_{i j}^{v(n-1)}$, obtém-se uma nova distribuição de tensão $\Delta \sigma_{i j}^{n}$ na seção, como mostrado na figura abaixo. 


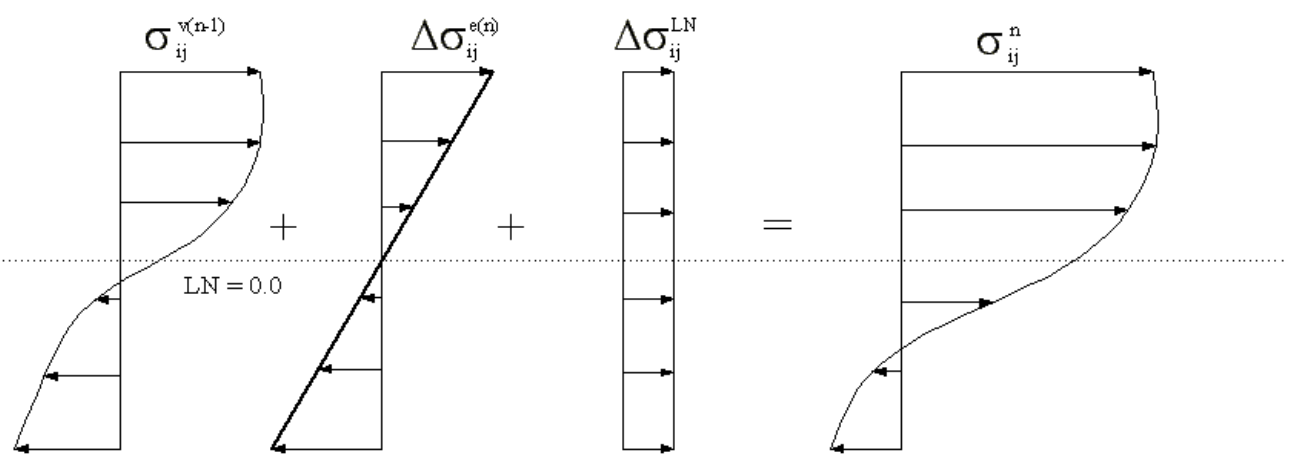

Figura 7.5 - Distribuição de tensão resultante em uma seção

Procede-se do mesmo modo para as armaduras, sendo que o incremento de tensão nas armaduras é dado por:

$$
\Delta \sigma_{i j}^{S(L N)}=E_{S} \Delta \varepsilon_{i j}^{L N} \delta_{i j}
$$

No caso do modelo de dano, calcula-se um estado de tensão elástico $\sigma_{i j(n)}^{e}$ através do estado de deformação total $\varepsilon_{i j}^{n}$.

Verifica-se então o modelo constitutivo para todos os pontos ao longo da espessura e calcula-se a nova normal resultante dada por:

$$
N_{C A}=\int_{-t / 2}^{t / 2} \sigma_{i j}^{C} d x_{3}+\sum_{n=1}^{N s} \sigma_{i j}^{S(n)} \delta_{i j} A_{s(n)}
$$

Fazendo a mudança de coordenadas, conforme anteriormente feita e a integração numérica chegamos a:

$$
N_{C A}=\frac{t}{2} \sum_{i g=1}^{N g} \sigma_{i j}^{C} W_{i g}+\sum_{n=1}^{N s} \sigma_{i j}^{S(n)} \delta_{i j} A_{s(n)}
$$

Considera-se que a posição da linha neutra em uma dada direção está correta quando o valor absoluto da normal resultante nessa seção é menor que:

$$
N_{C A} \leq \text { tol. } f_{c k} \cdot t
$$




\subsection{PROCEDIMENTO INCREMENTAL}

Definido o carregamento total, divide-se o mesmo em vários incrementos, que podem ser de diferentes tamanhos, definindo-se para cada incremento $\mathbf{i}$, o coeficiente $\beta_{\mathbf{i}}$ de multiplicação do carregamento total.

$$
\Delta M^{e(i)}=\beta_{i} M^{e}
$$

Procedendo desta forma, o novo incremento de carga pode ter seu tamanho definido de acordo com a performance obtida no incremento anterior, se foram necessárias muitas iterações para se obter o equilíbrio escolhe-se um menor valor do coeficiente multiplicativo. Logo, em trechos com forte não-linearidade, quanto menores os incrementos de carga, melhor será aproximada a curva não-linear. Para cada incremento de carga pode-se definir seu tamanho, sua tolerância e o número máximo de iterações admitido.

Passa-se ao incremento seguinte se o critério de convergência do incremento anterior foi verificado, em caso contrário o cálculo é interrompido, mesmo se ainda houver outros incrementos definidos a fazer.

\subsection{PROCESSO ITERATIVO}

No caso de não-linearidade física, procura-se o equilíbrio em um dado incremento de cargas através do Método de Newton-Raphson modificado, que considera a matriz $[S]$ (equação 4.18.a) constante e igual à obtida na primeira iteração do primeiro incremento, como mostrado na figura. O procedimento está mostrado no trabalho de CHAVES(1997).

Os valores dados na figura representam:

$(1 / r)^{n}$ é o vetor de curvaturas da iteração n

$\Psi^{n}$ é o vetor de resíduo da iteração n 


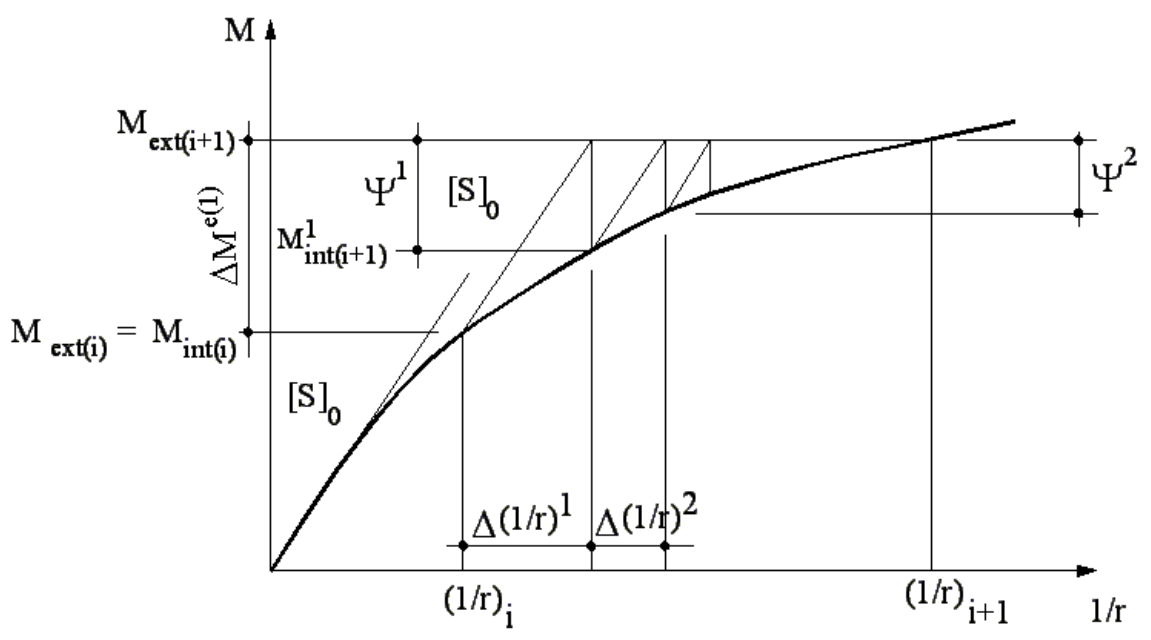

Figura 7.6 - Método de Newton-Raphson modificado

A tolerância no critério de convergência deve ser tal que dê resultados coerentes, sem a necessidade de realizar muitas iterações. Assim, tolerâncias muito folgadas produzem resultados não confiáveis pois refletem falsos estados de equilíbrio, e tolerâncias muito apertadas elevam o esforço computacional, fazendo iterações desnecessárias.

No caso de soluções divergentes, deve-se tentar reduzir os tamanhos dos incrementos e investigar a possibilidade de colapso da estrutura. Para soluções com convergência lenta, deve-se reduzir o tamanho dos incrementos, aumentar a tolerância de convergência ou usar o método Newton-Raphson padrão, recalculando a matriz $[S]$ a cada iteração, conforme procedimento sugerido nas conclusões e não disponível aqui.

\subsection{ROTEIRO DE SOLUÇÃO}

No início de cada iteração, tem-se um estado de tensão na estrutura que é estaticamente admissível, pois verifica as condições de equilíbrio da estrutura. Deve-se então verificar se em toda a estrutura o modelo constitutivo é obedecido. Assim, para uma iteração $\mathbf{n}$ de um incremento $\mathbf{i}$, tem-se um incremento de momentos elásticos $\left\{\Delta M^{e}\right\}$ para cada ponto do domínio, que é dado por: 


$$
\begin{aligned}
& \left\{\Delta M^{e}\right\}_{i}^{1}=\beta_{i}\{N\} \quad \text { se } n=1 \\
& \left\{\Delta M^{e}\right\}_{i}^{n}=[S]\left\{\Delta M^{0}\right\}_{j}^{n-1} \quad \text { se } n \geq 2
\end{aligned}
$$

onde $[S]$ e $\{N\}$ vem da equação (4.18).

Assim, considerando um ponto do domínio, deve-se proceder da seguinte maneira:

-Com $\left\{\Delta M^{e}\right\}_{i}^{n}$ calcula-se o incremento de curvaturas $\left\{\Delta(1 / r)^{e}\right\}_{i}^{n}$ e então para cada ponto ao longo da espessura determina-se o incremento de deformações $\left\{\Delta \varepsilon^{e}\right\}_{i}^{n}$ devido ao carregamento, obtendo-se então:

$$
\begin{aligned}
& \{1 / r\}_{i}^{n}=\{1 / r\}_{i}^{n-1}+\left\{\Delta(1 / r)^{e}\right\}_{i}^{n} \\
& \{\varepsilon\}_{i}^{n}=\{\varepsilon\}_{i}^{n-1}+\left\{\Delta(\varepsilon)^{e}\right\}_{i}^{n}
\end{aligned}
$$

-No caso do modelo elastoplástico, calcula-se também o incremento de tensões elásticas, para os pontos de Gauss e para as armaduras. Soma-se este ultimo ao estado de tensão verdadeiro da iteração anterior e calcula-se a tração nos pontos de Gauss. Considerando o modelo de dano do concreto, calcula-se as tensões elásticas $\left\{\sigma^{e}\right\}_{i}^{n}$. Verifica-se o modelo constitutivo, obtendo-se o vetor de tensão verdadeira $\left\{\sigma^{v}\right\}_{i}^{n}$ para o ponto em questão. Procedendo-se da mesma forma para todos os pontos de Gauss e armaduras, obtém-se uma nova distribuição de tensão ao longo da espessura.

-Verifica-se então a normal resultante nas três direções. Se alguma não for nula, estima-se a nova posição da linha neutra, verifica-se de novo os modelos constitutivos e calcula-se as novas normais resultantes. Este processo continua a'te que as normais sejam nulas (dentro da tolerância) nas três direções. Deste modo, obtém-se a verdadeira distribuição de tensões ao longo da espessura. 
-Obtém-se o valor dos momentos verdadeiros $\left\{M^{v}\right\}_{i}^{n}$ e o vetor de incremento de momentos verdadeiros $\left\{\Delta M^{v}\right\}_{i}^{n}$.

-Calcula-se o vetor de momentos residuais:

$$
\left\{\Delta M^{0}\right\}_{i}^{n+1}=\{\Psi\}_{n}^{i}=\left\{\Delta M^{e}\right\}_{i}^{n}-\left\{\Delta M^{v}\right\}_{n}^{i}
$$

-Para todos os pontos ao longo da espessura, verifica-se o critério de convergência. Segue-se o mesmo procedimento para todos os pontos do domínio. Se o critério não for verificado para algum ponto significa que o estado de tensão na estrutura é tal que verifica o modelo constitutivo em todos os pontos mas não conduz ao equilíbrio da estrutura. Assim, aplica-se $\{\Psi\}_{i}^{n}$ ao sistema como um campo de momentos iniciais, obtém-se um novo incremento de momentos elásticos e passa-se a iteração seguinte. Se o critério for verificado, passa-se ao incremento seguinte. O processo iterativo termina, portanto, quando o estado de tensão verificar ao mesmo tempo as condições de equilíbrio e o modelo constitutivo em todos os pontos da mesma. 


\section{EXEMPLOS}

\subsection{EXEMPLO 1:}

Para demonstrar a precisão na computação das integrais de domínio envolvendo o campo de momentos iniciais, iniciamos com a seleção de dois exemplos simples com solução exata conhecida: campos de momento constante e de momento linear aplicados em domínios retangulares.

Vamos inicialmente considerar um domínio retangular $\underline{\mathbf{L}} \times \underline{\mathbf{B}}$ ( $\mathrm{L}$ na direção $\mathrm{x}_{1}$ ) sobre o qual é aplicado um campo de momentos iniciais $M_{11}^{0}=1.0$ é aplicado. A placa é simplesmente apoiada ao longo dos lados de comprimento B e livre na outra direção. Foram computadas as distribuições de momentos e deslocamentos em toda a placa. A Tabela 1 mostra os valores computados ao longo de $\mathrm{x}_{1}$. Os valores exatos dos momentos são zero, portanto os valores obtidos representam os erros, demonstrando que a técnica é apurada. Deslocamentos também são mostrados, confirmando a precisão da formulação.

Tabela 8.1 - Momentos e Deslocamentos devidos a $\mathrm{M}_{11}=1.0$, eixo $\mathrm{x}_{2}=\mathrm{B} / 2$

\begin{tabular}{|c|c|c|c|c|}
\hline $\mathrm{X}_{1} / \mathrm{L}$ & \multicolumn{2}{|l|}{0.125} & \multicolumn{2}{|l|}{0.250} \\
\hline Solução & Exata & Num. & Exata & Num. \\
\hline $\mathrm{M}_{11} \mathrm{~L}^{2} / \mathrm{D}$ & 0.00 & $-2.70 \mathrm{E}-08$ & 0.00 & $-2.70 \mathrm{E}-08$ \\
\hline $\mathrm{WD} /\left(\mathrm{M}_{11} \mathrm{~L}^{2}\right)$ & 0.0546875 & 0.0546875 & 0.09375 & 0.09375 \\
\hline $\mathrm{X}_{1} / \mathrm{L}$ & \multicolumn{2}{|l|}{0.375} & \multicolumn{2}{|l|}{0.500} \\
\hline Solução & Exata & Num. & Exata & Num. \\
\hline $\mathrm{M}_{11} \mathrm{~L}^{2} / \mathrm{D}$ & 0.00 & $-2.70 \mathrm{E}-08$ & 0.00 & $-2.70 \mathrm{E}-08$ \\
\hline $\mathrm{WD} /\left(\mathrm{M}_{11} \mathrm{~L}^{2}\right)$ & 0.1171875 & 0.1171875 & 0.125000 & 0.125 \\
\hline
\end{tabular}


O erro máximo relativo obtido para os deslocamentos foi de 5.38E-7.

O segundo teste efetuado consiste na aplicação de um campo de momentos iniciais linear variando de zero para $\mathrm{x}_{1}=0$ até 1.0 para $\mathrm{x}_{1}=\mathrm{L}$, isto é $M_{11}^{0}=X_{1} / L$.

Como estamos usando aproximações quadráticas, respostas aproximadas eram esperadas para este exemplo. Deslocamentos e momentos são dados na Tabela 2, para pontos ao longo do eixo $\mathrm{x}_{2}$ passando pelo centro da placa na direção B. Os valores exatos do momento são zero, portanto os valores são os erros computados.

Tabela 8.2 - - Momentos e deslocamentos devido a $\mathrm{M}_{11}=\mathrm{X}_{1} / \mathrm{L}$

\begin{tabular}{|l|l|l|l|l|}
\hline $\mathrm{X}_{2} / \mathrm{B}$ & \multicolumn{3}{|l|}{0.005} & \multicolumn{2}{l|}{0.0125} \\
\hline Solução & Exata & Num. & Exata & Num. \\
\hline $\mathrm{M}_{11} \mathrm{~L}^{2} / \mathrm{D}$ & 0.00 & $2.33 \mathrm{E}-02$ & 0.00 & $4.00 \mathrm{E}-06$ \\
\hline $\mathrm{WD} /\left(\mathrm{M}_{11} \mathrm{~L}^{2}\right)$ & 0.02375 & 0.02375 & 0.054687 & 0.0546875 \\
\hline $\mathrm{X}_{2} / \mathrm{B}$ & 0.5000 & & \multicolumn{2}{|l|}{} \\
\hline Solução & Exata & Num. & & \\
\hline $\mathrm{M}_{11} \mathrm{~L}^{2} / \mathrm{D}$ & 0.00 & $2.80 \mathrm{E}-08$ & & \\
\hline $\mathrm{WD} /\left(\mathrm{M}_{11} \mathrm{~L}^{2}\right)$ & 0.125 & 0.125 & & \\
\hline
\end{tabular}

Na tabela 8.2 podemos notar que os deslocamentos mostraram grande precisão (erro 2.7E-8). Ao contrário, momentos próximos da borda são afetados pela aproximação dos valores de contorno. Para o nó $\mathrm{X}_{2} / \mathrm{B}=0.005$, com índice entre a distância ao contorno e o comprimento do elemento de contorno dado por 0.04, a precisão não é satisfatória, enquanto que para um índice correspondente de 0.10 a precisão é muito boa (4.0E-6). Devemos então recomendar que para a descrição do campo de momentos iniciais, não sejam usados nós muito próximos ao contorno. $\mathrm{O}$ mínimo valor da distância deste ponto ao contorno deve ser, conforme nossos resultados, de 0.100 do comprimento do elemento de contorno.

Como uma conclusão inicial, podemos dizer que é eficiente a transformação proposta para as integrais de domínio na análise de placas. Testes futuros mais completos poderão confirmar a estabilidade desta formulação. 


\subsection{EXEMPLO 2:}

Placa quadrada de lado $\mathrm{L}$, simplesmente apoiada no contorno, com carga uniformemente distribuída.

O contorno é discretizado em 20 elementos, o contorno da região carregada, que compreende toda a placa, é discretizado em 8 elementos.São analisadas diversas relações entre a espessura $h$ da placa e o seu lado L. São analisados também as condições de apoios "hard" (rotação nula na direção do plano vertical perpendicular ao contorno) e "soft" (momento nulo na direção do plano vertical perpendicular ao contorno).

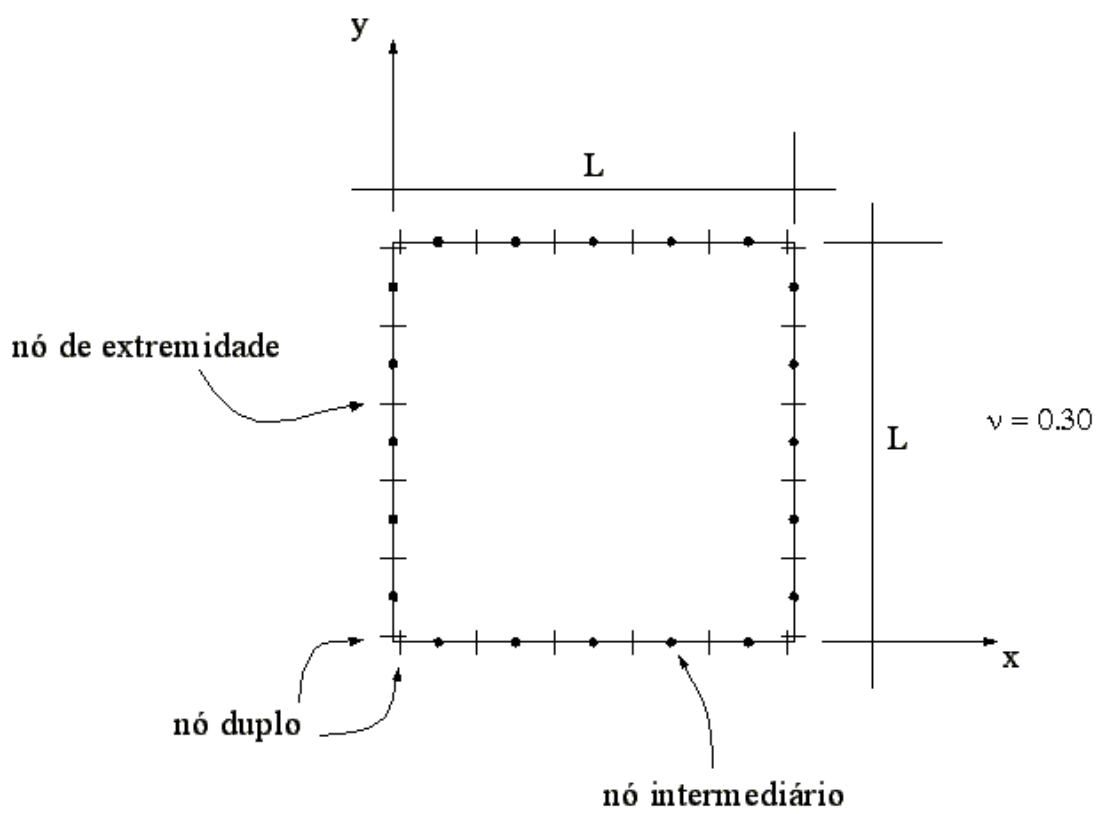

Figura 8.1 - Placa simplesmente apoiada no contorno 
Tabela 8.3 - Deslocamentos transversais w sobre a linha central da placa

\begin{tabular}{|c|l|l|l|l|l|l|}
\hline \multirow{2}{*}{$\mathrm{H} / \mathrm{L}$} & \multicolumn{5}{|c|}{ W.(100D) } \\
\cline { 2 - 7 } & \multicolumn{1}{|c|}{ X/L } & \multicolumn{1}{|c|}{$0.1 \mathrm{~L}$} & \multicolumn{1}{|c|}{$0.2 \mathrm{~L}$} & \multicolumn{1}{|c|}{$0.3 \mathrm{~L}$} & \multicolumn{1}{c|}{$0.4 \mathrm{~L}$} & \multicolumn{1}{c|}{$0.5 \mathrm{~L}$} \\
\hline \multirow{2}{*}{0.01} & MEC & 0.1316 & 0.2463 & 0.3338 & 0.3888 & 0.4064 \\
\cline { 2 - 7 } & Ribeiro & 0.132 & 0.246 & 0.334 & 0.388 & 0.406 \\
\hline \multirow{2}{*}{0.1} & MEC & 0.1386 & 0.2583 & 0.3490 & 0.4051 & 0.4241 \\
\cline { 2 - 7 } & Ribeiro & 0.139 & 0.258 & 0.349 & 0.405 & 0.424 \\
\hline \multirow{2}{*}{0.2} & MEC & 0.1597 & 0.2945 & 0.3951 & 0.4569 & 0.4478 \\
\cline { 2 - 7 } & Ribeiro & 0.160 & 0.295 & 0.395 & 0.457 & 0.478 \\
\hline \multirow{2}{*}{0.25} & MEC & 0.1756 & 0.3217 & 0.4298 & 0.4958 & 0.5180 \\
\cline { 2 - 7 } & Ribeiro & 0.176 & 0.322 & 0.430 & 0.496 & 0.518 \\
\hline \multirow{2}{*}{0.3} & MEC & 0.1950 & 0.3547 & 0.4718 & 0.5431 & 0.5672 \\
\cline { 2 - 7 } & Ribeiro & 0.195 & 0.355 & 0.472 & 0.543 & 0.567 \\
\hline \multicolumn{2}{|c|}{ Teoria Clássica } & 0.132 & 0.246 & 0.334 & 0.388 & 0.406 \\
\hline
\end{tabular}

A tabela 8.3 fornece os deslocamentos transversais w na linha central da placa correspondente a $y=\mathrm{L} / 2$, para condição de apoio "hard”, para diversas relações $\mathrm{h} / \mathrm{L}$ e compara-os com os valores da Teoria Clássica de Kirchhoff. Nota-se que para placas de pequena espessura, com $\mathrm{h} / \mathrm{L}=0.01$ os valores são coincidentes, a medida que a espessura da placa aumenta eles vão divergindo, chegando esta diferença a 39.7\% para uma relação $\mathrm{h} / \mathrm{L}=0.30$. Os resultados são comparados com os obtidos por RIBEIRO(1976), este usando a Teoria de Kirchhoff, aqui empregada a de Reissner. Foi adotado valor da constante $\alpha=0.50$ em todos os casos. 
Tabela 8.4 - Momentos Mxx sobre a linha central da placa

\begin{tabular}{|c|l|l|l|l|l|l|}
\hline \multirow{2}{*}{$\mathrm{H} / \mathrm{L}$} & \multicolumn{6}{|c|}{$\mathrm{M}_{\mathrm{xx}}\left(10 / \mathrm{q} . \mathrm{L}^{2}\right)$ sobre o eixo $\mathrm{y}=\mathrm{L} / 2$} \\
\cline { 2 - 7 } & $\mathrm{X} / \mathrm{L}$ & $0.1 \mathrm{~L}$ & $0.2 \mathrm{~L}$ & $0.3 \mathrm{~L}$ & $0.4 \mathrm{~L}$ & \multicolumn{1}{c|}{$0.5 \mathrm{~L}$} \\
\hline \multirow{2}{*}{0.01} & MEC & 0.2091 & 0.3433 & 0.4237 & 0.4658 & 0.4789 \\
\cline { 2 - 7 } & Ribeiro & 0.209 & 0.343 & 0.424 & 0.466 & 0.479 \\
\hline \multirow{2}{*}{0.1} & MEC & 0.2097 & 0.3442 & 0.4249 & 0.4673 & 0.4804 \\
\cline { 2 - 7 } & Ribeiro & 0.210 & 0.344 & 0.425 & 0.467 & 0.480 \\
\hline \multirow{2}{*}{0.2} & MEC & 0.2112 & 0.3465 & 0.4287 & 0.4717 & 0.4847 \\
\cline { 2 - 7 } & Ribeiro & 0.211 & 0.347 & 0.429 & 0.472 & 0.485 \\
\hline \multirow{2}{*}{0.3} & MEC & 0.2137 & 0.3517 & 0.4346 & 0.4785 & 0.4924 \\
\cline { 2 - 7 } & Ribeiro & 0.214 & 0.352 & 0.435 & 0.479 & 0.492 \\
\hline \multicolumn{2}{|c|}{ Teoria Clássica } & 0.209 & 0.343 & 0.424 & 0.466 & 0.479 \\
\hline
\end{tabular}

A tabela 8.4 fornece os momentos Mxx na linha central da placa correspondente a y=L/2, para condição de apoio "hard”, para diversas relações $h / L$ e compara-os com os valores da Teoria Clássica de Kirchhoff. Nota-se que para placas de pequena espessura, com $\mathrm{h} / \mathrm{L}=0.01$ os valores são coincidentes, a medida que a espessura da placa aumenta eles vão divergindo, chegando esta diferença a $2.80 \%$ para uma relação $h / L=0.30$.Notase portanto que os valores dos momentos são bem menos influenciáveis pela espessura da placa que os deslocamentos transversais w Os resultados são comparados com os obtidos por RIBEIRO(1976).

Tabela 8.5 - Valores de w, Mxx e Myy para relação h/L=0.10 e condições “soft” e "hard" de contorno, sobre a linha central da placa

\begin{tabular}{|c|c|c|c|c|c|c|}
\hline \multirow{3}{*}{$\mathrm{X} / \mathrm{L}$} & \multirow{2}{*}{\multicolumn{2}{|c|}{ W. $\frac{(100 \mathrm{D})}{\left(\mathrm{qL}^{4}\right)}$}} & \multirow{3}{*}{$\mathrm{M}_{\mathrm{xx}}$} & 10 & \multirow{2}{*}{$\mathrm{M}_{\mathrm{yy}}$} & \multirow{2}{*}{$\frac{10}{\left(q L^{2}\right)}$} \\
\hline & & & & $\left(\mathrm{qL}^{2}\right)$ & & \\
\hline & "SOFT" & "HARD" & & "HARD" & "SOFT" & "HARD" \\
\hline 0.10 & 0.1521 & 0.1386 & 0.2517 & 0.2097 & 0.1949 & 0.1707 \\
\hline 0.20 & 0.2811 & 0.2583 & 0.3806 & 0.3442 & 0.3307 & 0.3048 \\
\hline 0.30 & 0.3780 & 0.3490 & 0.4573 & 0.4249 & 0.4303 & 0.4017 \\
\hline 0.40 & 0.4377 & 0.4051 & 0.4979 & 0.4673 & 0.4904 & 0.4605 \\
\hline 0.50 & 0.4578 & 0.4241 & 0.5105 & 0.4804 & 0.5105 & 0.4804 \\
\hline
\end{tabular}

As diferenças entre os valores das condições de apoio "soft” e "hard” aumentam à medida que o ponto se aproxima do contorno. Para os valores do deslocamento 
transversal $\mathrm{w}$ estas diferenças ficam entre 7.36\% e 8.88\%, para valores de Mxx as diferenças permanecem entre 5.90\% e 16.69\% enquanto que os valores de Myy diferem entre $5.90 \%$ e $12.42 \%$. A condição de apoio “hard” é mais rígida e conduz a menores valores do deslocamento transversal $\mathrm{w}$.

\subsection{EXEMPLO 3:}

Placa quadrada apoiada nos quatro cantos com carga uniformemente distribuída

Analisa-se a mesma placa do exemplo 2, agora sem apoio ao longo do contorno e com os cantos apoiados em pilares O contorno é discretizado em 20 elementos, o contorno da região carregada, que compreende toda a placa, é discretizado em 8 elementos. Tal problema foi analisado por PAIVA(1987) utilizando Kirchhoff.

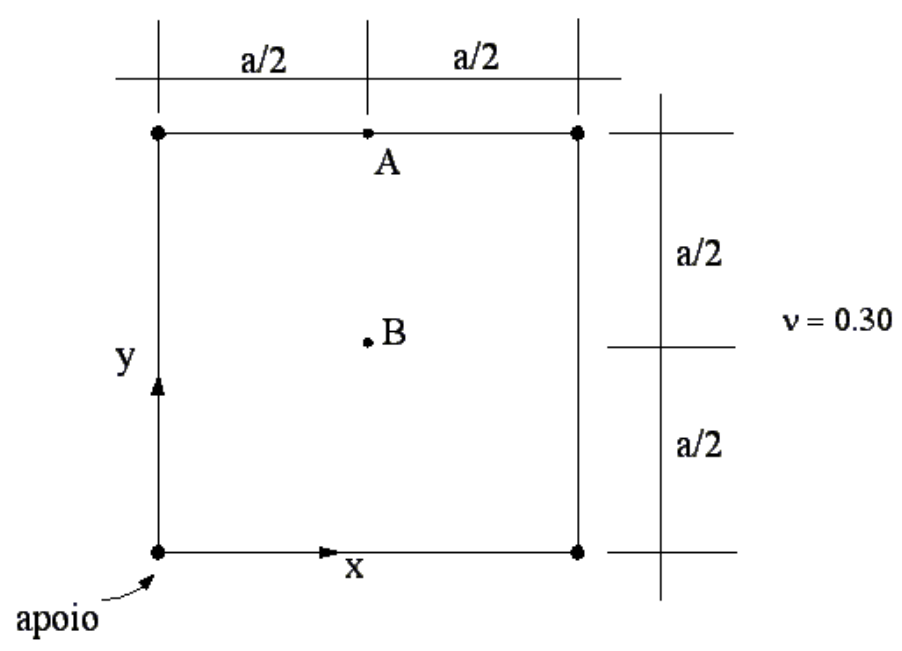

Figura 8.2 - Placa simplesmente apoiada nos quatro cantos 
Tabela 8.6 - Valores dos deslocamentos e esforços obtidos pela solução exata (TIMOSHENKO), por PAIVA e pelo MEC utilizando Reissner.

\begin{tabular}{|c|c|c|c|}
\hline VALORES & MEC - 20 Elem & PAIVA - 24 Elem & TIMOSHENKO \\
\hline $\mathrm{W}_{\mathrm{B}} /\left(\mathrm{qa}^{4} / \mathrm{D}\right)$ & 0.0228 & 0.0229 & 0.0249 \\
\hline $\mathrm{M}_{\mathrm{XXB}} /\left(\mathrm{qa}^{2}\right)$ & 0.109 & 0.109 & 0.109 \\
\hline $\mathrm{M}_{\mathrm{YYB}} /\left(\mathrm{qa}^{2}\right)$ & 0.109 & 0.109 & 0.109 \\
\hline $\mathrm{W}_{\mathrm{A}} /\left(\mathrm{qa}^{4} / \mathrm{D}\right)$ & 0.0151 & 0.0147 & --- \\
\hline $\mathrm{M}_{\mathrm{XXA}} /\left(\mathrm{qa}^{2}\right)$ & 0.131 & 0.129 & 0.1404 \\
\hline
\end{tabular}

\subsection{EXEMPLO 4:}

Placa quadrada simplesmente apoiada no contorno e com carga vertical uniformemente distribuída na linha $\mathrm{y}=\mathrm{a} / 2$

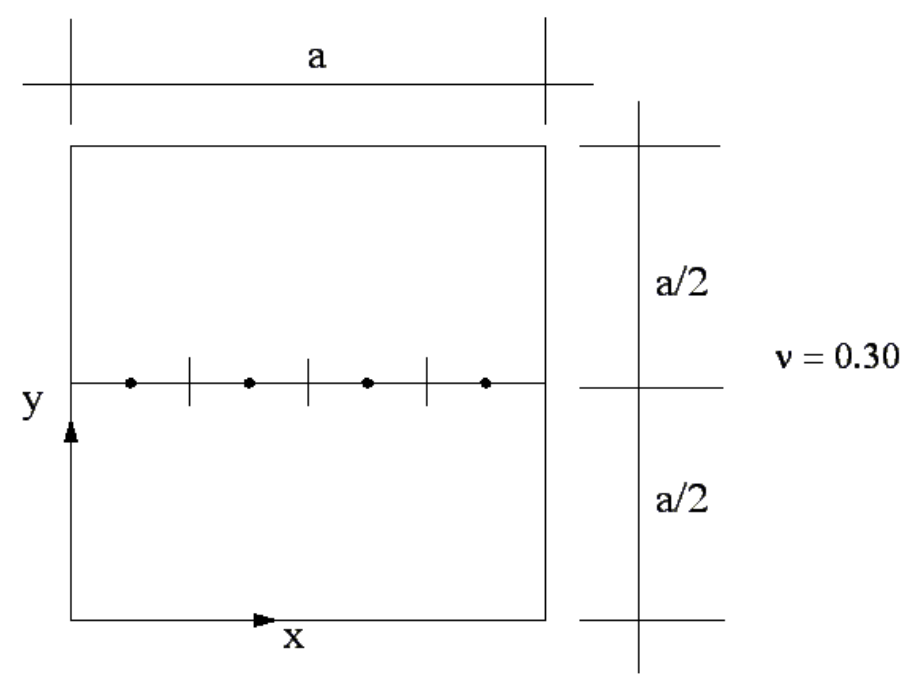

Figura 8.3 - Placa com linha de carga distribuída linearmente

A placa quadrada de lado a , simplesmente apoiada no contorno, com condição de apoio "hard", relação h/a=0.25, coeficiente de Poisson=0.30, com linha de carregamento constante na posição $\mathrm{y}=\mathrm{a} / 2$. O contorno é discretizado em 20 elementos. A linha de carga foi discretizada em 4 elementos. 
As soluções obtidas para o deslocamento transversal w, momentos Mxx e Myy são apresentadas nos gráficos juntamente com os resultados exatos calculados por TIMOSHENKO(1970). A precisão dos resultados foi excelente.

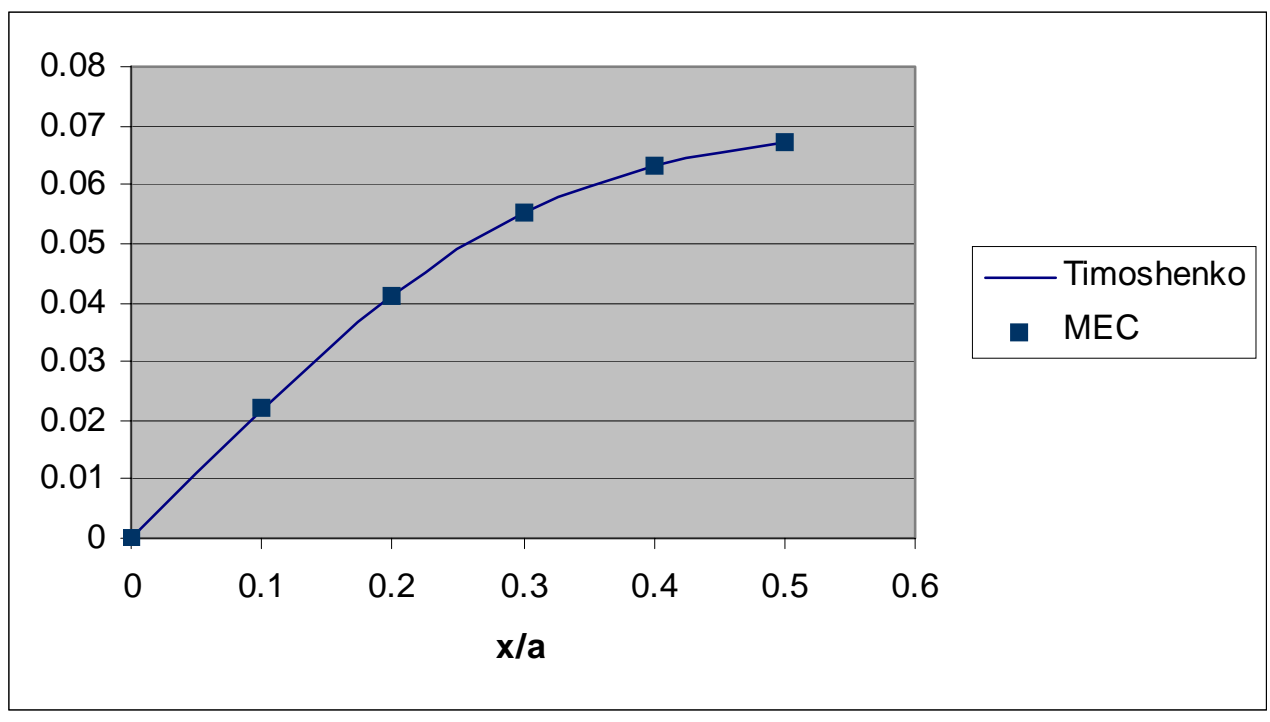

Figura 8.4 - Deslocamento transversal $w /\left(q a^{3} / D\right)$ na linha $y=a / 2$

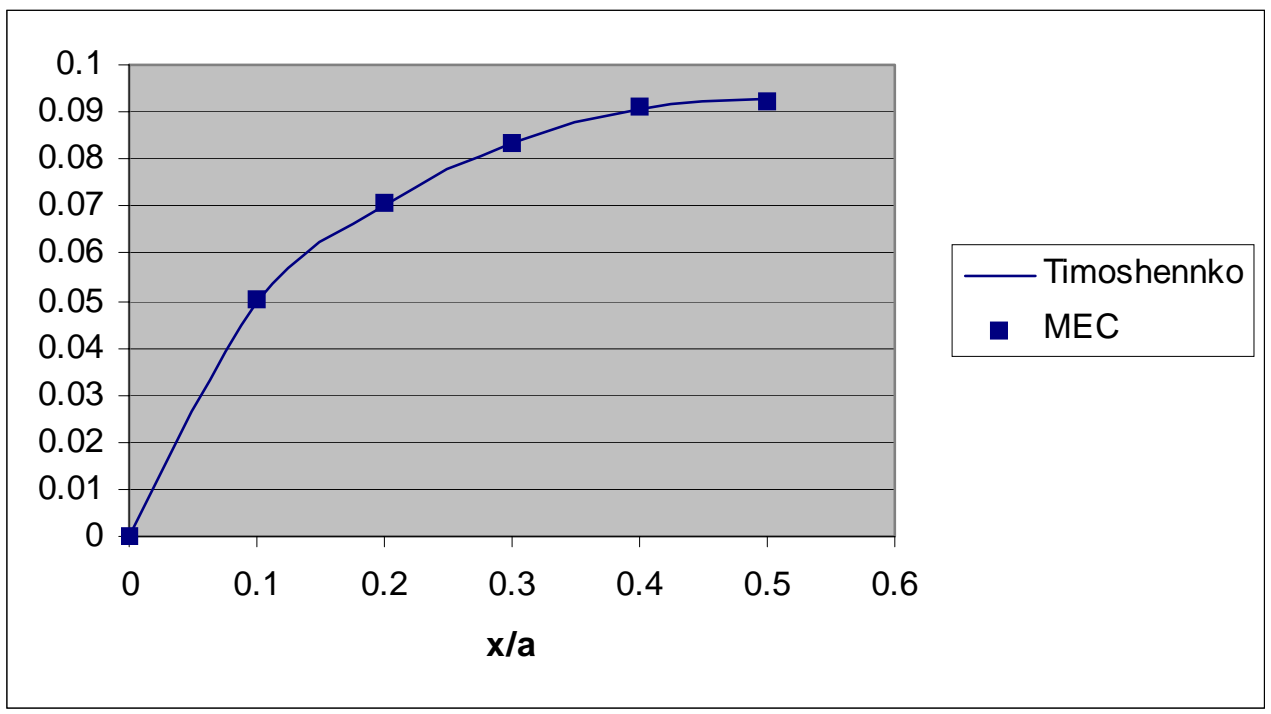

Figura 8.5 - Momento fletor $M_{x x} / q a$ na linha $y=a / 2$ 


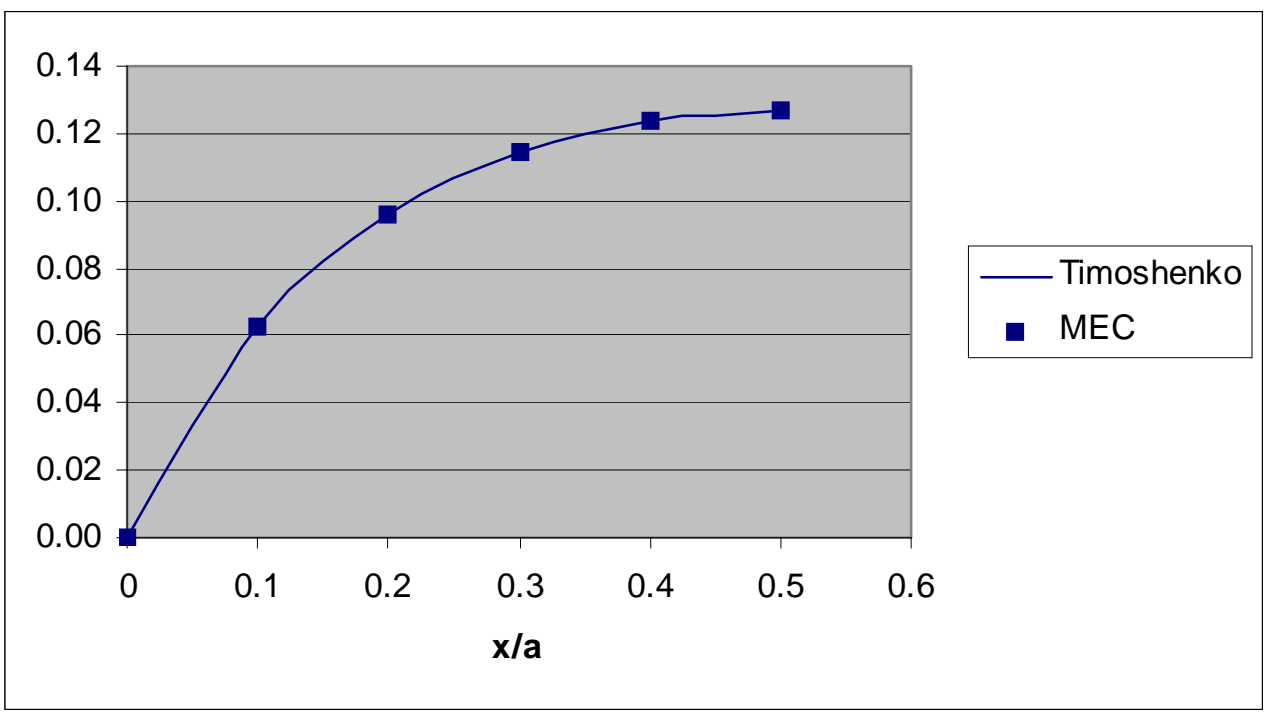

Figura 8.6 - Momento fletor $M_{y y} /$ qa na linha $y=a / 2$

\subsection{EXEMPLO 5:}

Placa quadrada simplesmente apoiada no contorno e com carga vertical uniformemente distribuída em toda a laje - análise do problema não-linear

Apresenta-se a seguir a análise não-linear de uma placa quadrada já ensaiada por CAMPOS(2000). Trata-se de laje quadrada de vão $400 \mathrm{~cm}$, altura $7 \mathrm{~cm}$, altura útil $6.1 \mathrm{~cm}$, armadura longitudinal $\phi 5.0 \mathrm{~mm}$ cada $20 \mathrm{~cm}$ nas duas direções, módulo de elasticidade do aço $20530 \mathrm{kN} / \mathrm{cm}^{2}$, parâmetro de endurecimento $2000 \mathrm{kN} / \mathrm{cm}^{2}$, tensão inicial de escoamento do aço $70 \mathrm{kN} / \mathrm{cm}^{2}$, resistência à compressão característica do concreto 20.7Mpa, módulo de elasticidade do concreto $1730 \mathrm{kN} / \mathrm{cm}^{2}$, coeficiente de Poisson 0.25 (adotado), carga distribuída $6.50 \mathrm{kN} / \mathrm{m}^{2}$.

O contorno da laje é discretizado em 20 elementos, o domínio é discretizado em 120 células, o contorno da região carregada(toda a placa) é discretizado em 8 elementos. 
Tabela 8.7- Deslocamento transversal w no centro da placa

\begin{tabular}{|c|c|c|}
\hline Carga $\left(\mathrm{kN} / \mathrm{m}^{2}\right)$ & CAMPOS(2000) & MEC -120 células \\
\hline 0.00 & 0.00 & 0.00 \\
\hline 0.25 & 0.71 & 0.60 \\
\hline 0.50 & 1.35 & 1.21 \\
\hline 0.75 & 1.93 & 1.89 \\
\hline 1.00 & 2.63 & 2.66 \\
\hline 1.25 & 3.35 & 3.57 \\
\hline 1.50 & 3.99 & 4.66 \\
\hline 1.75 & 4.82 & 5.89 \\
\hline 2.00 & 5.82 & 7.40 \\
\hline 2.25 & 8.20 & 9.31 \\
\hline 2.50 & 20.85 & 11.48 \\
\hline 2.75 & 24.96 & 14.70 \\
\hline 3.00 & 31.35 & 17.01 \\
\hline 3.50 & 40.85 & 22.05 \\
\hline 4.00 & 45.99 & 29.70 \\
\hline 4.50 & 51.57 & 36.25 \\
\hline 5.00 & 57.24 & 43.02 \\
\hline 5.50 & 63.16 & 51.21 \\
\hline 6.00 & 70.57 & 59.13 \\
\hline 6.50 & 75.05 & 67.41 \\
\hline
\end{tabular}

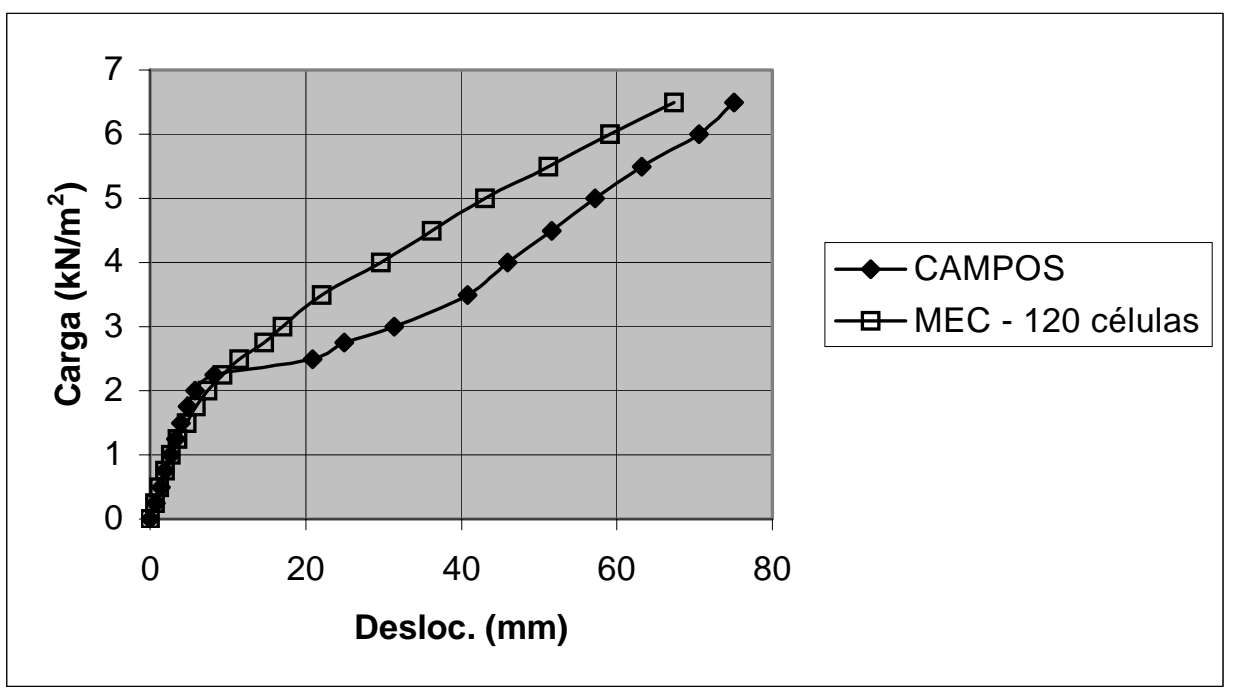

Figura 8.7 - Gráfico carga x deslocamento transversal w para o ponto central da laje 


\section{CONCLUSÕES}

Este trabalho estudou a formulação do método dos elementos de contorno para a análise não-linear de pavimentos de concreto armado. A teoria utilizada foi a de Reissner, que mostrou-se eficiente tanto para placas esbeltas quanto para as moderadamente espessas, uma vez que considera as deformações por cisalhamento.

Foram utilizados campos de momentos iniciais aplicados apenas em pontos internos, alguns relativamente próximos ao contorno.As integrais que envolvem as células de domínio foram aperfeiçoadas, eliminando-se as aproximações através de séries. Foi desenvolvida uma formulação para a análise de placas vinculadas a estruturas quaisquer em seu domínio, com o uso de cargas aplicadas: pontuais, em pequenas áreas ou em linhas. O acoplamento MEC/MEF foi empregado utilizando-se modelos simples, porém robustos.O sistema de equações algébricas foi otimizado com a utilização da técnica dos mínimos quadrados.

Uma primeira conclusão interessante a ser mencionada de acordo com o trabalho é a respeito da qualidade da integração dos elementos de contorno e das células internas. Com relação à integração dos elementos de contorno, mostrou-se que a utilização de sub-elementação garante precisão no sistema algébrico e a solução é sempre precisa. Pode-se inclusive mostrar perturbações dos esforços internos quando o ponto se aproxima do contorno, evidenciando a influência da aproximação dos valores de contorno.

Com relação à aproximação do campo de momentos inicial mostrou-se a conveniência de utilizar apenas pontos internos evitando-se assim o tratamento de 
integrais de contorno com núcleos bastante complexos. Além disso, foram utilizadas as funções primitivas das soluções fundamentais o que permitiu uma fácil transformação das integrais sobre os domínios das células em integrais sobre o contorno das mesmas, sem a presença de singularidades importantes. Todos os termos que correspondiam a efeitos do campo de momentos iniciais resultaram em integrais precisas e com núcleos bastante simples. Formas analíticas de integração para este caso são fáceis de serem obtidas o que pode, em tese, melhorar ainda mais as respostas numéricas. Mostrou-se também que as transformações feitas combinadas com modelos de integração precisos podem levar a uma estimativa muito precisa dos efeitos dos momentos iniciais. Para os casos clássicos onde os efeitos são conhecidos (momento aplicado constante por exemplo, chegou-se a precisão de $10^{-8}$. É interessante lembrar que a utilização dos núcleos originais da solução fundamental de Reissner-Mindlin leva a diferentes aproximações polinomiais (expansões) cuja integração numérica é sempre prejudicada, principalmente para os segmentos próximos dos pontos de colocação.

Outro ponto a ser enfatizado desta contribuição é a respeito da combinação MEC/MEF para a análise de placas enrijecidas. Observou-se que cuidados precisam ser tomados e também que devem ser evitados o uso e compatibilização de valores que possam ter a determinação de coeficientes correspondentes difícil devido às complexidades envolvidas. Por exemplo, foi retirada da combinação qualquer acoplamento que envolvesse o grau de liberdade rotação. A razão para isso é a não definição de rotação em extremidade de linha de momentos lineares aplicados. Para evitar tal singularidade é necessário que se use nós não extremos o que vai trazer um complicador para o acoplamento. A alternativa seria a distribuição dos momentos em linha numa superfície de dimensões calculadas em função da largura efetiva dos elementos lineares. Assim, não sendo possível a determinação de rotações devido a momentos em linha, eliminou-se o acoplamento deste grau de liberdade, chegando-se portanto a um modelo simples porém bastante eficiente. As rotações não compatibilizadas ficam melhor aproximadas quando se refina a malha. As rotações do eixo da barra são apenas compatibilizadas entre barras. 
No prosseguimento do trabalho podem ser considerados:

- $\quad$ Utilização de matriz tangente, atualizada a cada iteração, visando maior rapidez na convergência do processo;

- $\quad$ Utilização de outros modelos constitutivos dos materiais.

- $\quad$ Consideração da não-linearidade física também sobre os elementos de viga;

- $\quad$ Introdução do efeito de membrana, estudando-se o caso membranaflexão;

- $\quad$ Uso de modelos de dano anisotrópicos. 


\section{BIBLIOGRAFIA}

ALIABADI, M.H.; BREBBIA, C.A.; RASHED, Y.F. On the evaluation of the stresses in the BEM for Reissner plate-bending problems. Appl. Math. Modelling, v.21, p. 155-163, March, 1997.

ALTIERO, N.J.; SIKARSKIE, D.L. A boundary integral method applied to plates of arbitrary plan form. Computers \& Structures. v.9, p. 163-168, 1978.

ÁLVARES, M.S. Estudo de um modelo de dano para o concreto: formulação, identificação paramétrica e aplicação com emprego do Método dos Elementos Finitos. Dissertação (Mestrado). São Carlos. Escola de Engenharia de São Carlos, Universidade de São Paulo, 1993.

ARGYRIS, J.H.; KELSET, S. Energy theorems and structural analysis. London, butteworths, 1960 .

BACARJI, E. Aplicação do Método dos Elementos de Contorno à Análise de Pavimentos. São Carlos. 160p. Tese (Doutorado) - Escola de Engenharia de São Carlos, Universidade de São Paulo, 2001.

BARCELOS, C.S.; SILVA, L.H.M. A boundary element formulation for Mindlin's plate model. In: BREBBIA, C.A.; VENTURINI, W.S. eds. BETECH87, Comp. Mech. Publ., 1987.

BARRET, K.E.; ELLIS, S. An exact theory of elastic plates. Int. J. Solids Struct. v.24(9), p. 859-880, 1988.

BATOZ, J.L.; BATHE, K.J.; HO, L.W. A study of three-node triangular plate bending elements. Int. J. Num. Meth. Eng., v.15, p. 1771-1812, 1980.

BETTI, E. Teoria dell'elasticità. Il Nuovo Ciemento. p. 7-10, 1872.

BEZINE , G.P.; GAMBY, D.A. A new integral equation formulation for plate bending problems. In: Brebbia, C.A. ed. Recent advances in BEM. p. 327-342, Pentech Press, 1978. 
BEZINE, G.P. A boundary integral equation method for plate flexure with conditions inside domain. Int. J. Num. Meth. Engng, n.17, p. 1647-1657, 1981.

BEZINE, G.P. Boundary integral formulation for plate flexure with arbitrary boundary conditions. Mech. Res. Comm., v.5, n.4, p. 197-206, 1978.

BREBBIA, C.A. The boundary element method for engineers. London: Pentech Press, 1978.

BUSSAMRA, F.L.S. Equações constitutivas do concreto baseadas na mecânica do dano contínuo. Dissertação (Mestrado). Escola Politécnica da Universidade de São Paulo, 1993.

CERVENKA, V. Inelastic Finite Element analysis of reinforced concrete panels under in plane loads. Ph.D thesis. University of Colorado, 1970.

CHEN, A.C.T.; CHEN, W.F. Constitutive Relations for concrete. Journal of Engineering Mechanics Division, ASCE, v.101, n. n.EM4, p. 465-481, August, 1975.

CHENG, S. Elastic theory of plates and a refined theory. J. Appl. Mech., n.46, p. 644-650, 1959.

CHUEIRI, L.H.M. Formulação do Método dos Elementos de Contorno para análise elastoplástica de placas. São Carlos. Tese (Doutorado) - Escola de Engenharia de São Carlos, Universidade de São Paulo, 1994.

DEBBIH, M.; EL-ZAFRANY, A.; FADHIL, S. Boundary Element analysis of thick Reissner plates in bending. Engineering Analysis, v.14, p. 159-169, 1995.

DEBBIH, M.; EL-ZAFRANY, A.; FADHIL, S. An efficient approach for Boundary Element analysis of thick Reissner plates in bending. Computers \& Structures, v.56, p. 565-576, 1995.

EL-ZAFRANY, A.; FADHIL, S.; DEBBIH, M. An efficient approach for boundary element bending analysis of thin and thick plates. Computers \& Structures, 56, p. 565-576, 1995.

FERNANDES, G.R. O método dos elementos de contorno aplicado à análise não-linear de placas. São Carlos. Dissertação (Mestrado). - Escola de Engenharia de São Carlos, Universidade de São Paulo, 1988. 
FORBES , D.J.; ROBINSON, A.R. Numerical analysis of elastic plates and shallow shells by na integral equation method. University of Illinois Structural Research Series Report, n.346, 1969.

FUNG, Y.C. Foundations of Solid mechanics. New Jersey, Prentice-Hall, 1965.

HANSEN, E.B. Numerical solution of integro-differential and singular integral equations for plate bending problems. J. of Elasticity. v.6(1), p. 39-56, 1976.

HARTLEY, G.A.; ABDEL-AKHER, A. Boundary integration and interpolation procedures for plate bending. Int. Num. Meth. Engng. v. 28(6), p. 1389-1408, 1989.

HARTMANN, F. Kirchhoff Plates. Ind: BREBBIA, C.A., ed., Boundary Elements X, v.3, C.M. Publ., p. 409-423, 1988.

HU, H.; SCHNOBRICH, W.C. Nonlinear finite element analysis of reinforced concrete plates and shells under monotonic loading. Comput. \& Struct., n.38, p. 637651, 1991.

JASWON, M.A.; MAITI, M.; SYMM, G.J. Numerical biharmonic analysis and some applications. Int. J. Solids Structures, n. 3, p. 309-332, 1967.

JOFRIET, J.C.; McNEICE, G.M. Finite element analysis of reinforced concrete slabs. J. Struct. Div., ASCE, n.97, p. 785-806, Mar 1971.

KACHANOV, L.M. Time of the rupture process under creep conditions. T.V.Z. Akad. S.S.R. Tech. Nauk., 8, p.26-31, 1958.

KARAM, V.J. Análise de flexão de placas pelo MEC incluindo não-linearidade física. Rio de Janeiro. Tese (Doutorado) - Universidade Federal do Rio de Janeiro, COPPE, 1992.

KARAM, V.J. Aplicação do Método dos Elementos de Contorno à Teoria de Reissner para flexão de placas. Rio de Janeiro. Dissertação (Mestrado) - Universidade Federal do Rio de Janeiro, COPPE, 1986

KARAMI, G.; ZARRINCHANG, J.; FOROUGHI, B. An efficient analytical treatment of boundary integrals in direct boundary element analysis of plate bending problems. In: BREBBIA, C. A., DOMINGUEZ, J.; PARIS, F., eds. Boundary elements XIV, v.2, 1992.

KAYSIKADELIS, J.T.; YOTIS, A.J. A new boundary element solution of thick plates modelled by Reissner's theory. Eng. Anal. Bound. Element.,1993. 
KIRCHHOFF, G. Uber das gleichgwicht und die bewegung einer elastischen scleibe. J. Math.,v.40, p. 51-58, 1850.

KRAJCINOVIC, D.; FONSEKA, G.Y. The continuous damage theory of brittle materials. J. Appl. Mech., v.48, p. 816-824, December, 1981.

KUPRADZE, V.D. Potencial methods in the theory of elasticity. Israel Progr. Sci. Transl., 1965.

LEVINSON, M. An accurate, simple theory of statics and dynamics of elastic plates. Mech. Res. Comm. v.7(6), p. 343-350, 1980.

MARCZAK, R.J.; CREUS, G.J. Direct evaluation of singular integrals in boundary element analysis of thick plates. Engineering Analysis with a Boundary Element, 26, p. 653-665, 2002.

MARTINELLI, D.A.O.; MONTANARI, I.; SAVASSI, W. Placas elásticas Publicação 026/86. São Carlos, 113p. - Escola de Engenharia de São Carlos, Universidade de São Paulo, 1986.

MAZARS, J. Application de lá mécanique de l'endommagement au comportement non lineaire et à la rupture du béton de structure. Thèse de Doctorat ès sciences, Université Paris 6, 1984.

MIKHLIN, S.G. Integral equations. London, Pergamon Press, 1957.

MINDLIN, R.D. Influence of rotatory inertia and shear on flexural motions of isotropic, elastic plates. J. Appl. Mech, v.13, p. 31-38, 1951.

MOSHAIOV, A.; VORUS, W.S. Elasto-plastic bending analysis by a Boundary Element Method with initial plastic moments. Int. J. Solids Struct. v.22(11), p. 12131229, 1986.

MUSKHELISHVILI, N. I. Some basic problems of mathematical theory of elasticity. Noordhoof, Gronigen Holand, 1953.

NORDGREN, R.P. A bound on the error in plates theory. Quart. Appl. Math. V.28(4), p. 587-595, 1971.

NORDGREN, R.P. A bound on the error in Reissner's theory plates. Quart. Appl. Math. v.29, p. 551-556, 1972.

OLIVEIRA NETO, L. Uma formulação do Método dos Elementos de Contorno com três parâmetros nodais para placas delgadas e suas aplicações a problemas de 
engenharia estrutural. São Carlos. Tese (Doutorado). - Escola de Engenharia de São Carlos, Universidade de São Paulo, 1998.

PAIVA, J.B. Formulação do Método dos Elementos de Contorno para flexão de placas e suas aplicações em engenharia de estruturas. São Carlos. Tese (Doutorado) Escola de Engenharia de São Carlos, Universidade de São Paulo, 1987.

PALERMO JR, L. A análise de placas e o Método dos Elementos de contorno. Campinas, 142p. Tese de Livre Docência - Universidade Estadual de Campinas, 2000.

PALERMO JR, L. Plate bending anlysis using the classical or the ReissnerMindlin models. Engineering Analysis with a Boundary Elements, 2003 (to appear).

PANC, V. Theories of elastic plates. International Publishing, Noordhoff, 1975.

PAPADOPOULOS, P.; TAYLOR, R.L. A Triangular Element Based on Reissner - Mindlin Plate Theory. Int. J. Num. Meth. Engrg, n.30, p. 1029-1049, 1990.

PROENÇA, S.P.B. Sobre modelos matemáticos do comportamento do concreto: análise crítica e contribuições. São Carlos. Tese (Doutorado) - Escola de Engenharia de São Carlos, Universidade de São Paulo, 1988.

RAO, P.S.; SUBRAHMANYAM, B.V. Trisegmental moment-curvature relations for reinforced concrete members. Proc. Of the American Concrete Institute, n.70, p. 346-351, May 1973.

RASHED, Y.F.; ALIABADI, M.H.; BREBBIA, C.A. Hypersingular boundary element formulation for Reissner Plates. Int. J. Solids \& Structures, 35, p. 2229-2249, 1998.

REISSNER, E. On a generalization of some formulas of the theory of moderately thich elastic plates. Int. J. Solids Struct., v.23 (6), p. 711-717, 1987.

REISSNER, E. On small defletions of sheardeformable elastic plates. Comput. Meth. Mech. Engng., v.59, p. 227-233, 1986.

REISSNER, E. On the theory of transverse bending of elastic plates. J. Math. Physics. v.23, p.184-191, 1944.

REISSNER, E. The effect of tranverse shear deformation on the bending of plastic plates. Journal of Applied Mechanics, v.12, p. A69-A77, 1945. 
RIBEIRO, G.O. Sobre a formulação do Método dos Elementos de Contorno para a flexão de placas usando as hipóteses de Reissner. São Carlos, 266p. Tese (Doutorado) - Escola de Engenharia de São Carlos, Universidade de São Paulo, 1992.

RIBEIRO, G.O.; VENTURINI, W.S. Aplicação do Método dos Elementos de Contorno ao cálculo de placas através da teoria de Reissner. MECOM-89. Anais do X Congresso Ibero-Latino-Americano sobre métodos computacionais em engenharia - II Encontro Nacional de Mecânica Computacional. Porto, Portugal, 1989.

RIZZO, F.J. An integral approach to boundary value problems of classical elastostatics. Quart. Appl. Math., v.25, n.1, p. 83-92, 1967.

RYTCHTER, Z. An improved error estimate for Reissner's plate theory. Int. J. Solids Struct. v. 24(5), p. 537-544, 1988 .

SALERNO, V.L.; GOLDBERG, M.A. Effect of shear deformations on the bending of retangular plates. J. Appl. Mech. p. 54-58, 1960.

SILVA, N.A. Aplicação do Método dos Elementos de Contorno às placas com enrijecedores utilizando a Teoria de Reissner. São Carlos. Tese (Doutorado) - Escola de Engenharia de São Carlos, Universidade de São Paulo, 1996.

SOUTHWELL, R.V. Relaxation methods in theoretical physics. Oxford University Press. London, 1946.

STERN, M.A. A general boundary integral formulation for the numerical solution of plate bending problems. Int. J. Solids Struct. v.15, p. 769-782, 1979.

TESSLER, A.; HUGHES, T.J.R. A Three-node Mindlin Plate Element with Improved Transverse Shear. Comp. Meth. Appl. Engng., n.50, p. 71-101, 1985.

TIMOSHENKO, S.P.; WOYNOWSKY-KRIEGER, S. Theory of plates and shells. New York, Mc Graw Hill Book C ompany, Inc., 1959.

TURNER, M.J. et al. Stiffnes and deflection analysis of complex structures. J. Aero. Science, v.23, p. 805-823, 1956.

WEEËN, F.V. Aplication of Boundary Integral Equation Method to Reissner's plate model.Int. J. Num. Meth. Engng.,v.18(1), p. 1-10, 1982.

WEEËN, F.V. Aplication of Direct Boundary Element Method to Reissner's plate model. Boundary Element Method in Engineering, Procedings of $4^{\text {th }}$. International Seminar Southampton, 1982. 
WESTPHAL JR, T.; ANDRA, H.; SCHMACK, E. Some solutions for Kirchhoff, Reissner and Mindlin plate and a unified BEM formulation. Engineering Analysis with a boundary Elements, 25, p. 129-139, 2001.

XIAO-YAN, L.; KUANG-MAO, H.; XIUXI, W. Geometrically nonlinear analysis of a Reissner type plate by the Boundary Element Method. Computers \& Structures, v.37(6), p. 911-916, 1990. 


\section{APÊNDICE - SOLUÇÕES FUNDAMENTAIS}

$$
\begin{aligned}
& u_{\alpha \beta}^{*}=\frac{1}{8 \pi D(1-v)}\left\{\left[8 B-(1-v)(2 \ln z-1) \delta_{\alpha \beta}\right.\right. \\
& \left.-[8 A+2(1-v)] r,{ }_{\alpha} r,_{\beta}\right\} \\
& u_{\alpha 3}^{*}=-u_{3 \alpha}^{*} \frac{1}{8 \pi D}(2 \ln z-1) r r,,_{\alpha} \\
& u_{33}^{*}=\frac{1}{8 \pi D(1-v) \lambda^{2}}\left[(1-v) z^{2}(\ln z-1)-8 \ln z\right]
\end{aligned}
$$

onde A e B são funções de $\mathrm{z}$ dadas por:

$$
\begin{aligned}
& A(z)=K_{0}(z)+\frac{2}{z}\left[K_{1}(z)-\frac{1}{z}\right] \\
& B(z)=K_{0}(z)+\frac{1}{z}\left[K_{1}(z)-\frac{1}{z}\right]
\end{aligned}
$$

sendo $\mathbf{K}_{\mathbf{0}}(\mathbf{z})$ e $\mathbf{K}_{\mathbf{1}}(\mathbf{z})$ funções de Bessel modificadas de ordem inteira, podendo ser calculadas através de expansões polinomiais dadas por ABRAMOWITZ E STEGUN (1965). A expansão de A(z) para pequenos argumentos mostra que esta função é contínua, enquanto a expansão de $\mathbf{B}(\mathbf{z})$ apresenta singularidade do tipo $\ln \mathbf{z}$. r é a distância entre o ponto fonte $\xi$ e o ponto de deslocamento $\mathbf{x}$. 


$$
\begin{aligned}
& u_{\alpha \beta, \gamma}^{*}=-\frac{1}{4 \pi r D(1-v)}\left[\left(4 A+4 z K_{1}+1-v\right) r,_{\gamma} \delta_{\alpha \beta}\right. \\
& \left.-2\left(2 z K_{1}+8 A+1-v\right) r,{ }_{\alpha} r,_{\beta} r,{ }_{\gamma}+(4 A+1-v)\left(r,{ }_{\alpha} \delta_{\beta \gamma}+r,_{\beta} \delta_{\alpha \gamma}\right)\right] \\
& u_{\alpha \beta, \beta}^{*}=-\frac{r, \alpha}{2 \pi r D} \\
& u_{\alpha 3, \beta}^{*}=-u_{3 \alpha, \beta}^{*}=\frac{1}{8 \pi D}\left[\delta_{\alpha \beta}(2 \ln z-1)+2 r,{ }_{\alpha} r,_{\beta}\right. \\
& u_{\alpha 3, \alpha}^{*}=-u_{3 \alpha, \alpha}^{*}=\frac{\ln z}{2 \pi D} \\
& u_{33, \alpha}^{*}=-\frac{r,_{\alpha}}{8 \pi r^{2} D(1-v)}\left[(1-v) z^{2}(2 \ln z-1)-8\right] \\
& p_{\alpha \beta}^{*}=-\frac{1}{4 \pi r}\left[\left(4 A+2 z K_{1}+1-v\right)\left(\delta_{\alpha \beta} r,_{n}+r,_{\beta} n,{ }_{\alpha}\right)\right. \\
& \left.+(4 A+1+v) r,{ }_{\alpha} n,{ }_{\beta}-2\left(8 A+2 z K_{1}+1-v\right) r,_{\alpha} r,_{\beta} r,_{n}\right] \\
& p_{\alpha 3}^{*}=\frac{\lambda^{2}}{2 \pi}\left(B n_{\alpha}-A r,{ }_{\alpha} r,_{n}\right) \\
& p_{3 \alpha}^{*}=-\frac{(1-v)}{8 \pi}\left[\left(2 \frac{(1+v)}{(1-v)} \ln z-1\right) n_{\alpha}+2 r,{ }_{\alpha} r,_{n}\right] \\
& p_{33}^{*}=-\frac{1}{2 \pi r} r,_{n}
\end{aligned}
$$

onde $\mathbf{r}, \mathbf{n}$ é a derivada de $\mathbf{r}$ na direção normal no ponto $\mathbf{x}$.

$$
\begin{aligned}
& u_{\alpha \beta \gamma}^{*}=\frac{1}{4 \pi r}\left[\left(4 A+2 z K_{1}+1-v\right)\left(\delta_{\beta \gamma} r,_{\alpha}+\delta_{\alpha \gamma} r,_{\beta}\right)\right. \\
& \left.-\left(16 A+4 z K_{1}+2-2 v\right) r,_{\alpha} r,_{\beta} r,_{\gamma}+(4 A+1+v) \delta_{\alpha \beta} r,_{\gamma}\right]
\end{aligned}
$$




$$
\begin{aligned}
& u_{\alpha \beta 3}^{*}=-\frac{(1-v)}{8 \pi}\left\{\left[2 \frac{(1+v)}{(1-v)} \ln z\right] \delta_{\alpha \beta}+2 r,{ }_{\alpha} r,_{\beta}\right\} \\
& u_{3 \beta \gamma}^{*}=\frac{\lambda^{2}}{2 \pi}\left(B \delta_{\gamma \beta}-A r,_{\gamma} r,_{\beta}\right) \\
& u_{3 \beta 3}^{*}=\frac{1}{2 \pi r} r{ }_{\beta} \\
& p_{\alpha \beta \gamma}^{*}=\frac{D(1-v)}{4 \pi r^{2}}\left\{\left(4 A+2 z K_{1}+1-v\right)\left(\delta_{\alpha \gamma} n_{\beta}+\delta_{\gamma \beta} n_{\alpha}\right)\right. \\
& +(4 A+1+3 v)\left(\delta_{\alpha \beta} n_{\gamma}\right)-\left(16 A+6 z K_{1}+z^{2} K_{0}+2-2 v\right)\left[\left(n_{\alpha} r,_{\beta}+n_{\beta} r,_{\alpha}\right) r,_{\gamma}\right. \\
& \left.+\left(\delta_{\gamma \alpha} r,_{\beta}+\delta_{\gamma \beta} r,_{\alpha}\right) r,_{n}\right]-\left(16 A+4 z K_{1}+2+2 v\right)\left(\delta_{\alpha \beta} r,{ }_{\gamma} r,_{n}+n_{\gamma} r,_{\alpha} r,_{\beta}\right) \\
& \left.\left.+\left(96 A+32 z K_{1}+4 z^{2} K_{0}+8-8 v\right)\right) r,_{\alpha} r{ }_{\beta} r{ }_{\gamma} r{ }_{n}\right\} \\
& p_{\alpha \beta 3}^{*}=\frac{D(1-v) \lambda^{2}}{4 \pi r}\left[\left(2 A+z K_{1}\right)\left(n_{\alpha} r,_{\beta}+n_{\beta} r,{ }_{\alpha}\right)-\left(8 A+2 z K_{1}\right)\right) r,_{\alpha} r,_{\beta} r,_{n} \\
& \left.+2 A \delta_{\alpha \beta} r,_{n}\right] \\
& p_{3 \beta \gamma}^{*}=-\frac{D(1-v) \lambda^{2}}{4 \pi r}\left[\left(2 A+z K_{1}\right)\left(\delta_{\alpha \beta} r,_{n}+n_{\beta} r,_{\gamma}\right)+2 A n_{\gamma} r,_{\beta}\right. \\
& \left.\left.-\left(8 A+2 z K_{1}\right)\right) r,{ }_{\gamma} r,_{\beta} r,_{n}\right] \\
& p_{3 \beta 3}^{*}=\frac{D(1-v) \lambda^{2}}{4 \pi r^{2}}\left[\left(z^{2} B+1\right) n_{\beta}-\left(z^{2} A+2\right) r,{ }_{\beta} r,_{n}\right] \\
& r_{\alpha \beta}^{*}=-\frac{(1-v)}{8 \pi}\left\{\delta_{\alpha \beta}\left[\frac{2(1+v)}{(1-v)} \ln z-1\right]+2 r,{ }_{\alpha} r,_{\beta}\right. \\
& \left.-\frac{4 v}{(1-v) \lambda^{2} r^{2}}\left(2 r,_{\alpha} r,_{\beta}-\delta_{\alpha \beta}\right)\right\} \\
& r_{3 \beta}^{*}=\frac{r,{ }_{\beta}}{2 \pi r}
\end{aligned}
$$




$$
\begin{aligned}
& t_{\alpha \beta \gamma}^{*}=\frac{1}{8 \pi r}\left\{\left[8 A+4 z K_{1}+2(1-v)\right]\left(\delta_{\alpha \gamma} r,_{\beta}+\delta_{\beta \gamma} r,_{\alpha}\right)\right. \\
& \left.+2 r,_{\gamma} \delta_{\alpha \beta}(4 A+1+v)-2 r,_{\alpha} r,_{\beta} r,_{\gamma}\left[16 A+4 z K_{1}+2(1-v)\right]\right\} \\
& t_{3 \beta \gamma}^{*}=\frac{\lambda^{2}}{2 \pi}\left(B \delta_{\beta \gamma}-A r,_{\beta} r,_{\gamma}\right) \\
& v_{\alpha, \beta}^{*}=\frac{r^{2}}{128 \pi D}\left[\delta_{\alpha \beta}(1-v)(4 \ln z-5)+2(4 \ln z-3) r,_{\alpha} r,_{\beta}\right] \\
& v_{3, \beta}^{*}=\frac{r r,{ }_{\beta}}{128 \pi D(1-v) \lambda^{2}}\left[32(2 \ln z-1)-z^{2}(1-v)(4 \ln z-5)\right] \\
& w_{\alpha, \beta}^{*}=\frac{r}{64}\left\{( 4 \operatorname { l n } z - 3 ) \left[(1-v)\left(r,_{\beta} n,_{\alpha}+r,{ }_{\alpha} n,_{\beta}\right)+(1-3 v) \delta_{\alpha \beta} r,_{n}\right.\right. \\
& \left.+4\left[(1-v) r,{ }_{\alpha} r,_{\beta}+v \delta_{\alpha \beta}\right] r,_{n}\right\}-\frac{v}{(1-v) \lambda^{2}} u_{\alpha \beta \gamma}^{*} n,_{\gamma} \\
& w_{3, \beta}^{*}=\frac{1}{8 \pi}\left[(2 \ln z-1) n,,_{\beta}+2 r,_{\beta} r,_{n}\right]-\frac{v}{(1-v) \lambda^{2}} u_{3 \beta \gamma}^{*} n,,_{\gamma}
\end{aligned}
$$

$\lim _{\varepsilon \rightarrow 0} \int_{\Gamma \varepsilon} t_{111}^{*}(\xi, x) d \bar{\Gamma}_{\varepsilon}(x)=\frac{1}{2 \pi}\left[(1-v) \operatorname{sen} \varphi+2(1+v)\left(\operatorname{sen} \varphi-\frac{\operatorname{sen}^{3} \varphi}{3}\right)\right]$

$\lim _{\varepsilon \rightarrow 0} \int_{\bar{\Gamma} \varepsilon} t_{112}^{*}(\xi, x) d \bar{\Gamma}_{\varepsilon}(x)=\frac{1}{2 \pi}\left[(1-v) \cos \varphi-\frac{2}{3}(1+v) \cos ^{3} \varphi\right]$

$\lim _{\varepsilon \rightarrow 0} \int_{\bar{\Gamma} \varepsilon} t_{121}^{*}(\xi, x) d \bar{\Gamma}_{\varepsilon}(x)=\frac{1}{2 \pi}\left[-(1-v) \cos \varphi-\frac{2}{3}(1+v) \cos ^{3} \varphi\right]$

$\lim _{\varepsilon \rightarrow 0} \int_{\bar{\Gamma} \varepsilon} t_{122}^{*}(\xi, x) d \bar{\Gamma}_{\varepsilon}(x)=\frac{1}{2 \pi}\left[(1-v) \operatorname{sen} \varphi+\frac{2}{3}(1+v) \operatorname{sen}^{3} \varphi\right]$

$\lim _{\varepsilon \rightarrow 0} \int_{\bar{\Gamma} \varepsilon} t_{221}^{*}(\xi, x) d \bar{\Gamma}_{\varepsilon}(x)=\frac{1}{2 \pi}\left[-(1-v) \operatorname{sen} \varphi+\frac{2}{3}(1+v) \operatorname{sen}^{3} \varphi\right]$ 


$$
\begin{aligned}
& \lim _{\varepsilon \rightarrow 0} \int_{\bar{\Gamma} \varepsilon} t_{111}^{*}(\xi, x) d \bar{\Gamma}_{\varepsilon}(x)=\frac{1}{2 \pi}\left[-(1-v) \cos \varphi-2(1+v)\left(\cos \varphi-\frac{\cos ^{3} \varphi}{3}\right)\right] \\
& g_{\alpha \beta \gamma \theta}(\xi) \frac{1}{8}\left[(3-v)\left(\delta_{\alpha \gamma} \delta_{\theta \beta}+\delta_{\beta \gamma} \delta_{\theta \alpha}\right)-(1-3 v) \delta_{\alpha \beta} \delta_{\gamma \beta}\right] \\
& e_{\alpha \beta \gamma \theta}^{*}=-\frac{1}{8 \pi r^{2}}\left\{[ 1 6 A + 1 2 z K _ { 1 } + 2 ( 1 - v ) + 4 z ^ { 2 } K _ { 0 } ] \left(r,{ }_{\theta} r,{ }_{\beta} \delta_{\alpha \gamma}\right.\right. \\
& \left.+r,_{\theta} r,_{\alpha} \delta_{\beta \gamma}\right)+\left[16 A+4 z K_{1}+2(1-v)\right]\left(2 r,{ }_{\beta} r,{ }_{\gamma} \delta_{\alpha \theta}+2 r,{ }_{\alpha} r{ }_{\beta} \delta_{\gamma \theta}\right. \\
& \left.+2 r,{ }_{\gamma} r{ }_{\theta} \delta_{\alpha \beta}+2 r,{ }_{\alpha} r,{ }_{\gamma} \delta_{\beta \theta}+r,{ }_{\alpha} r,_{\theta} \delta_{\beta \gamma}+r,{ }_{\beta} r,_{\theta} \delta_{\alpha \gamma}\right) \\
& -(4 A+1-v)\left(2 \delta_{\gamma \theta} \delta_{\alpha \beta}+\delta_{\alpha \theta} \delta_{\gamma \beta}+\delta_{\beta \theta} \delta_{\gamma \alpha}\right) \\
& -\left(4 A+4 z K_{1}+1-v\right)\left(\delta_{\alpha \theta} \delta_{\beta \gamma}+\delta_{\alpha \gamma} \delta_{\beta \theta}\right)-\left[96 A+32 z K_{1}+8(1-v)\right. \\
& \left.\left.+4 z^{2} K_{0}\right]\left(2 r,_{\alpha} r,_{\beta} r,_{\gamma} r,_{\theta}\right)+4 v \delta_{\alpha \beta}\left(2 r,{ }_{\gamma} r,_{\theta}-\delta_{\gamma \theta}\right)\right\} \\
& e_{3 \beta \gamma \theta}^{*}=-\frac{\lambda^{2}}{2 \pi r}\left[\left(A+z K_{1}\right) r,{ }_{\theta} \delta_{\beta \gamma}-\left(4 A+z K_{1}\right) r,{ }_{\beta} r,_{\gamma} r{ }_{\theta}\right. \\
& \left.+A\left(r,{ }_{\gamma} \delta_{\beta \theta}+r,_{\beta} \delta_{\gamma \theta}\right)\right]
\end{aligned}
$$

